\title{
Towards understanding interchangeability of generic drugs
}

Citation for published version (APA):

Yu, Y. (2017). Towards understanding interchangeability of generic drugs. [Doctoral Thesis, Maastricht University]. Datawyse / Universitaire Pers Maastricht. https://doi.org/10.26481/dis.20170519yy

Document status and date:

Published: 01/01/2017

DOI:

10.26481/dis.20170519yy

Document Version:

Publisher's PDF, also known as Version of record

\section{Please check the document version of this publication:}

- A submitted manuscript is the version of the article upon submission and before peer-review. There can be important differences between the submitted version and the official published version of record.

People interested in the research are advised to contact the author for the final version of the publication, or visit the DOI to the publisher's website.

- The final author version and the galley proof are versions of the publication after peer review.

- The final published version features the final layout of the paper including the volume, issue and page numbers.

Link to publication

\footnotetext{
General rights rights.

- You may freely distribute the URL identifying the publication in the public portal. please follow below link for the End User Agreement:

www.umlib.nl/taverne-license

Take down policy

If you believe that this document breaches copyright please contact us at:

repository@maastrichtuniversity.nl

providing details and we will investigate your claim.
}

Copyright and moral rights for the publications made accessible in the public portal are retained by the authors and/or other copyright owners and it is a condition of accessing publications that users recognise and abide by the legal requirements associated with these

- Users may download and print one copy of any publication from the public portal for the purpose of private study or research.

- You may not further distribute the material or use it for any profit-making activity or commercial gain

If the publication is distributed under the terms of Article $25 \mathrm{fa}$ of the Dutch Copyright Act, indicated by the "Taverne" license above, 
Towards

understanding

$\diamond$ interchangeability

$\theta^{\ominus} \oslash \oslash^{\ominus}$ of generic drugs

$\theta_{0}^{(1)} \theta \theta_{\theta}^{\infty}$

$0 A \theta_{0}^{0} \theta_{0}^{\circ}$

$\theta \otimes \theta^{\circ} \theta 0$

$\theta^{\theta} \theta-\otimes \theta^{\otimes}$ Yang ru

$\rightarrow \theta 0 \theta 0 \theta^{\infty} \theta$

0
$\theta$$\theta^{\circ} \theta \theta \theta \theta \theta 0$

$\theta-\otimes \theta \theta \theta \theta=\theta$ 

To my Mother and Family 献给我的母亲和家人 
(C) copyright Yang Yu, Maastricht 2017

All rights reserved. No part of this publication may be reproduced,

stored in a retrieval system, or transmitted in any form or by any means, mechanically, by photocopying, recording, or otherwise, without the written permission of the author.

Printing: Datawyse | Universitaire Pers Maastricht

ISBN 9789461596833

Printing of this thesis was financially sponsored by Medicines Evaluation Board in the Netherlands. 


\title{
Towards understanding interchangeability of generic drugs
}

\author{
Dissertation \\ To obtain the degree of Doctor at the Maastricht University, \\ on the authority of the Rector Magnificus, \\ Prof. dr. Rianne M. Letschert \\ in accordance with the decision of the Board of Deans, \\ to be defended in public \\ On Friday 19 May 2017, at 14:00 hours
}

by

Yang Yu 


\section{Supervisor(s):}

Prof. dr. C. Neef, Maastricht University

Prof. dr. D. Burger, Radboud University Nijmegen Medical Centre

\section{Co-supervisor:}

Dr. M. Maliepaard, Medicines Evaluation Board, Utrecht

\section{Assessment committee:}

Prof. dr. A. Bast, Maastricht University (chairman)

Dr. M.H.L Christiaans, Maastricht University Medical Centre

Prof. dr. T. van Gelder, Erasmus Medical Center Rotterdam

Prof. dr. R.A.A. Mathôt, Academic Medical Center Amsterdam 


\section{Contents}

Chapter 1 General introduction

Chapter 2 Equivalence of generic medicines in general and immunosuppressants in particular - a regulatory opinion on switching of ciclosporin, tacrolimus and mycophenolate mofetil

Chapter 3 A comparison of the intrasubject variation of drug exposure between generic and brand-name drugs: a retrospective analysis of replicate design trials

Chapter 4 The cumulative impact of post-marketing variations on interchangeability of generic drugs: a model for assessing bioequivalence

Chapter 5 Interchangeability of gabapentin generic formulations in the Netherlands: a comparative bioavailability study

Chapter 6 Investigation into the interchangeability of generic formulations using immunosuppressants and a broad selection of medicines

Chapter 7 General discussion

Appendix I Summary

Samenvatting

Appendix II Acknowledgements

List of co-authors

List of publications

About the author

Valorisation Addendum 

Chapter

General introduction 



\section{HISTORY AND REGULATION OF GENERIC DRUGS}

According to the European Medicines Agency (EMA), "a generic medicine is a medicine that is developed to be the same as a medicine that has already been authorized (the 'reference medicine')" (1). A generic medicine contains the same active substance(s) quantitatively and qualitatively as the reference medicine, and it is used at the same dose(s) to treat the same disease(s) as the reference medicine. The demonstration of bioequivalence between the generic drug and the reference drug has been used worldwide as basic criterion for approval of a generic drug (2-4). A generic drug can only be marketed once the period of 'exclusivity' of the reference drug has expired, for instance, in Europe typically 10 years from the date of first authorization (1).

Back in the nineteenth century and early twentieth century, the drug industry often produced simple chemical products. Medicines were prepared at pharmacies or hospitals (5). At that time, there was no market exclusivity for a new drug product. In 1860, the Adulteration Act was passed in England and was the earliest legislation to regulate food and drugs. In 1906, the Pure Food and Drugs Act was passed in the United States (US) (6). The drug industry did not use the patent system, and thus there was no classification of drugs as brand name or generic. Medicines were nonproprietary or called 'ethical' pharmaceuticals. Without patent protection, any company could produce medicines, and that era laid the basis for the development of generic companies. From the 1930s onward, the pharmaceutical industry shifted toward the in-house development of medicines and started to develop proprietary medicines protected by patents. For instance, Terramycin ${ }^{\circledR}$ (Oxytetracycline) from Pfizer was patented and approved by the Food and Drug Administration (FDA) in 1950 (7). In the USA, the companies that owned the patent of brand-name drugs depicted the production of generic drugs as a form of counterfeiting, which had a large influence on prescriptions at clinics and pharmacies. The period 1930-1960 is also known as the pharmaceutical golden age (8).

In the USA, in 1962 Kefauver-Harris amendments to the Food, Drug and Cosmetic Act of 1938 required the proof of efficacy of all new medicines; it also included provisions for drugs produced before 1962 (9). In these amendments, generic companies were allowed to produce off-patent medicines that were available before 1962 and these products would be considered efficacious, without the need for new clinical studies, on the basis of a so-called "literature-based" New Drug Application (NDA). However, the scientific literature on the majority of brand-name drugs was not sufficient to support assumptions of efficacy and safety in order to gain approval of generic drugs via "literature-based" applications (10). From that moment, the number of generic drugs started to increase, but even in 1983 only 35\% of the top-selling drugs with expired patents had a generic drug and only $13 \%$ of all prescriptions written out in 1984 in the USA were for generic drugs (10). A milestone for generic drugs was in 1984, when the Drug Price Competition and Patent Term Restoration Act (known as the Hatch-Waxman Act) was approved by the US Congress $(11,12)$. The Act granted market exclusivity for a 
new drug product (i.e., as a brand-name drug) approved on or after October 10, 1962. In addition, the Hatch-Waxman Act allowed the FDA to approve marketing authorization for generic drugs released after 1962, based on the demonstration of bioequivalence with brand-name drugs, as support for the registration of medicinal products without the need for repeating efficacy and safety trials. Further, generic companies were allowed to start bioequivalence studies before the patent of the brand-name drug had expired. The concept of demonstrating bioequivalence with the brand-name drug is still applied for the registration of generic drugs. Today, almost all brand-name drugs have at least one generic drug, and in the last decade the relative number of prescriptions of generic drugs has increased to $84 \%$ in 2012 in the USA (13). In the Netherlands, the prescription of generic drugs increased to $70 \%$ in 2013 , the cost of which accounted for $16 \%$ of the total cost of medicines prescribed in that year (14).

Nevertheless, the companies that own brand-name drugs still try to influence the development of generic drugs in order to retain their market share (15). For example, Gaviscon (sodium alginate and potassium bicarbonate) in 2008 and Losec (omeprazole) in 2005. In 2008, it was reported that Reckitt Benckiser had created obstacles to block the development of a generic version of Gaviscon. Gaviscon had been registered without a generic name and its chemical composition was unknown. After the patent for Gaviscon expired in 1999, the company objected to generic versions in 2000, 2005, and 2006. In August 2006, a monograph on Gaviscon was issued, but there is still no generic version of Gaviscon available in Europe. In 2005, AstraZeneca was fined by the European Commission for providing European regulatory agencies with misleading information about the date of the patent for Losec, which delayed the development of a generic version of the drug. One strategy adopted by brand-name companies in order to retain the market share has been to merge with generic companies, e.g. Ciba-Geigy merged with Sandoz in 1996 to form Novartis, which resulted in the acquisition of the generic companies, e.g. Novartis and Hexal in 2005, or to form a partnership with generic companies, e.g. Pfizer, Aurobindo Pharma, and Claris Lifesciences in 2009. In the deal with Aurobindo, Pfizer acquired the rights to commercialize 55 solid oral-form generic drugs and five sterile injections. Pfizer also acquired the rights to 15 injections from Claris Lifesciences (16).

\section{DISTRUST OF GENERIC DRUGS}

From a health economic point of view, the prescription of generic drugs instead of brand-name drugs can reduce the financial burden on national healthcare systems and also promote patient access to medicines (17). Patients often can obtain a generic drug at a much lower price than the brand-name drug. The main reason behind the lower price of generic drugs is the lower investment in research and development, but the relatively simpler and faster registration process relative to that for the brand-name drug also has a role to a certain extent. For a drug containing a new chemical entity, a 
pharmaceutical company spends about 10-20 years on research and on the completion of the non-clinical and clinical studies required to demonstrate the efficacy and safety of the drug. It can take about a year to obtain marketing authorization. For instance, the EMA officially takes at least 210 days to approve a new drug, but the complexity and uncertainty surrounding a new drug may mean that the procedure is extended because of the requirement for additional information or explanations. For generic drugs, nonclinical and clinical studies are not needed, only a comparative bioavailability (mostly only a single-dose cross-over) study is requested, which dramatically saves pharmaceutical companies both time and money. However, possibly because of the low cost of generic drugs and the relatively simple registration requirements, patients and health professionals often express doubts about the quality of generic drugs and are reluctant to switch from brand-name to generic drugs (17-22). This distrust might also be a historical consequence of the use of the media by brand-name companies to influence the prescription of generic drugs in the twentieth century (5).

Currently, the issue regarding the quality and interchangeability of generic drugs (i.e., therapeutic equivalence) is still controversial. In the literature, the issue of switching from a brand-name drug to a generic drug most frequently concerned antiepileptic drugs, for which a higher rate of switching back to the brand-name drug and a higher relapse rate with generic drugs have been reported $(20,23,24)$. Furthermore, there are reports in the literature that drug exposure is different with generic and brand-name drugs (21). However, a systematic review of seizure control (25) and a study of clozapine (26) showed that treatment outcomes with generic drugs appeared to be the same as with brand-name drugs. For cardiovascular disease, the brand-name drugs were also shown not to be superior to generic drugs (27). Further concerns regarding the interchangeability of generic drugs in the literature have focused on the shortcomings of the current bioequivalence approach $(28,29)$, the impact of a different appearance of generic and brand-name drugs(30), and the influence of different excipients (e.g., formulations containing sorbitol) (31).

\section{Bioequivalence of generic drugs and interchangeability}

In 1984, the concept of bioequivalence was introduced for the registration of generic drugs in the USA. From the side of the European Commission, the first European guidance on bioequivalence, i.e. "Note for Guidance: Investigation of bioavailability and Bioequivalence", was issued in 1992 by the Committee of Proprietary Medicinal Products (CPMP)(32). Since then, this EU guidance for generic drugs has been regularly revised $(2,33)$. Currently, the core requirement is to conduct a single-dose bioequivalence study with healthy volunteers, to demonstrate that the generic drug has a rate and extent of absorption comparable with those of the brand-name drug (2). To demonstrate bioequivalence, the $90 \%$ confidence interval $(\mathrm{Cl})$ of the ratios between the gener- 
ic drug and the brand-name drug for the pharmacokinetic parameters Cmax and AUC are required to be within the $80-125 \%$ range.

Regulatory authorities have recognized that there are questions, challenges, and limitations to the bioequivalence concept, and effort has been made to improve the guidance and to cover the variety of drug formulations and drug properties currently available (34).

\section{Challenges and effort}

As the registration of a generic drug depends on a single-dose bioequivalence study in a relatively small population, concerns have been expressed about the study of generic drugs, such as the study population, the single dose design, the number of subjects included, and the evaluation of study outcome. As bioequivalence studies may be carried out with healthy volunteers instead of patients, a population that is obviously different from the population that actually uses the drug, patients and prescribers sometimes wonder whether the demonstration of bioequivalence in healthy volunteers is relevant to patients (35). In addition, as in clinical practice patients are often prescribed multipledose medications, the relevance of bioequivalence tested using a single-dose scheme can be questioned. Moreover, it is still debated whether bioequivalence is the same as therapeutic equivalence. To deal with these concerns and questions about bioequivalence, the guidelines from regulatory authorities have explained the requirements for a bioequivalence study. Relevant information can also be found in literature (35-37).

In the period 1990-2000, regulatory authorities started to take a look at the methodology used to establish bioequivalence $(38,39)$. The routinely applied bioequivalence approach is known as the average bioequivalence approach, by which bioequivalence is defined on the basis of the ratio of average drug exposure achieved with the generic drug and the brand-name drug. However, it cannot be excluded that drug exposure is different in individual patients treated with a generic or brand-name drug (39). In order to deal with this issue, two alternative approaches, i.e., individual bioequivalence and population bioequivalence, were developed by the FDA in 1999 and a draft guideline was launched (40). Compared with the average bioequivalence approach, individual and population bioequivalence approaches not only evaluate the average difference in generic and the brand-name drug exposure but also take into account the variation in the average difference in exposure among individuals (i.e., subject-by-formulation interaction variance). The individual bioequivalence method allows comparison of intra-subject variation with both generic and brand-name drugs and the subject-by-formulation interaction, whereas the population bioequivalence method assesses the total variability in pharmacokinetic parameters (such as AUC and $\mathrm{C}_{\max }$ ) in the population. Overall, these two new bioequivalence approaches were expected to improve the comparability of generic and brand-name drugs. Nevertheless, because of issues related to the masking effect, power and sample size determination, statistical procedures, and study design 
(41), the guideline for using individual and/or population bioequivalence method was withdrawn. In Chapter 3, our study outcome also supports the issue regarding the masking effect of the two methods.

In addition, as generic drugs demonstrate bioequivalence only to their brand-name drug, in theory different generic drugs of the same brand-name drug may not necessarily bioequivalent. If multiple generic drugs are available, switching between generic drugs can occur in clinical practice (e.g., in the Netherlands as a consequence of the 'preferentiebeleid' [preference policy]). Concerns about generic-generic drug interchangeability were already mentioned in the literature in 1996 (42). However, this issue was only picked up by the authorities $(43,44)$ and others $(18)$ in 2008 . Investigation of generic-generic drug interchangeability is also a topic of this thesis (Chapter 5 and 6 ).

Since 2000, in addition to dealing with the concerns of patients and health professionals about generic drugs, the authorities have also improved guidelines for generic drugs. For example, a tightened acceptance range of 90-111\% for AUC and/or Cmax has been required for narrow therapeutic index drugs since 2001 (45). The reason for this is that the effectiveness and safety of narrow therapeutic index drugs are strongly related and sensitive to the level of exposure to the drug. Thus, the tightened range of 90$111 \%$ for generic drugs with a narrow therapeutic index was required to reduce the variation that is considered acceptable between generic and brand-name drugs, thereby ensuring comparable treatment outcomes with either the generic drug or the brandname drug. Other examples of the improvements made are the Biopharmaceutics Classification System (BCS)-based biowaiver, which allows the registration of generic drugs without an in vivo bioequivalence study, and the wider acceptance range for the $90 \% \mathrm{Cl}$ for Cmax of highly variable drugs, in recognition of a high intra-subject variability $(2,34$, $46,47)$. In addition to the general guideline for the investigation of bioequivalence, both the FDA and the EMA have published product-specific guidelines for generic drugs, explaining the requirements and the reasoning underlying these requirements, in order to maintain the consistency of generic drug assessments $(48,49)$.

\section{AIM AND OUTLINE OF THE THESIS}

The aim of the studies described in this thesis was to investigate issues that may have an impact on the interchangeability of a generic drug and its brand-name drug from a regulatory and pharmacokinetic perspective (Part I), and also on the interchangeability of generic drugs (Part II). An additional aim was to provide recommendations for optimizing the regulation of generic drugs. 


\section{Part I Interchangeability of generic drugs and brand-name drugs}

In order to address public concern about generic drugs, and more specifically about immunosuppressants, Chapter 2 provides a regulatory opinion about generic drug substitutions in general, and for cyclosporine, tacrolimus, and mycophenolate mofetil in particular.

In clinical practice, differences in drug exposure observed in individual patients after they are switched from a brand-name drug to a generic drug have triggered concerns and doubts about generic drugs. The study described in Chapter 3 therefore investigated whether differences in total and peak drug exposure upon generic substitution are due to differences between formulations or to intra-subject pharmacokinetic variability of the active substance.

After drugs receive marketing approval, quality variations in the drug formulation are allowed. These are assessed by regulatory authorities on a case-by-case basis. It is conceivable that multiple critical variations in a drug made during the post-approval period may have a cumulative effect on the quality of the drug. For generic drugs, individual variations may not lead to significant differences in the formulation assessed by regulatory authorities, but the total series of variations combined may change the pharmacokinetic profile relative to that of the initial formulation that demonstrated bioequivalence with the brand-name drug. The accumulation of changes in quality may in theory affect bioequivalence with the brand-name drug. Consequently, the interchangeability of generic drugs may be questioned. In Chapter 4, we developed a risk assessment model to quantify the cumulative effect of multiple (post-marketing) quality variations in both generic and brand-name drugs, and to estimate the extent to which this risk affects conclusions about bioequivalence and therefore the interchangeability of generic drugs.

\section{Part II Interchangeability of generic drugs}

As multiple generic drugs are often available for a single brand-name drug, switching between generic drugs can occur in clinical practice. To date, bioequivalence between generic drugs has not been investigated clinically. In order to investigate the issue of generic-generic drug substitution, the bioequivalence study reported in Chapter 5 compared gabapentin exposure after the administration of the gabapentin brand-name drug and three generic drugs currently marketed in the Netherlands. The aims of this study were to assess substitution between gabapentin generic drugs and to validate an established inter-study comparison method $(43,44)$.

Lastly, the study presented in Chapter 6 investigated generic-generic drug interchangeability for a larger set of generic drugs, using the validated inter-study comparison method. We tested whether the conclusion drawn on the basis of the study presented in Chapter 5 can be extrapolated to other generic drugs beside generic gabapentin drugs. 


\section{REFERENCES}

(1) Questions and answers on generic medicines. (European Medicine Agency,, London, 2012).

(2) Guidline on the Investigation of Bioequivalence. (Committee for Medicinal Products for Human use (CHMP), European Medicine Agency,, London, 2010).

(3) Health Canada Guidance for Industry: Bioequivalence requirements-critical dose drugs. (ed. Branch, H.P.a.F.) (Health Canada, Ottawa, 2006).

(4) Guidance for Industry. Bioavailability and Bioequivalence. Studies for Orally Adminsitered Drug ProductsGeneral Considerations. US Department of Health and Human Services, Food and Drug Administration Center for Drug Evaluation and Research (CDER) (2003).

(5) Greene, J.A. What's in a name? Generics and the persistence of the pharmaceutical brand in American medicine. Journal of the history of medicine and allied sciences 66, 468-506 (2011).

(6) London, J. Tragedy, transformation, and triumph: comparing the factors and forces that led to the adoption of the 1860 Adulteration Act in England and the 1906 Pure Food and Drug Act in the United States. Food Drug Law J 69, 315-42, ii-iii (2014).

(7) Company History. <http://www.pfizer.com/about/history/all>. Accessed April 12016.

(8) The Pharmaceutical Golden Era: 1930-60. <https://pubs.acs.org/cen/coverstory/83/8325/8325 golden.html> (2005). Accessed April 12016.

(9) The Food, Drug and Cosmetic Act: amendments and regulations. Bulletin of the New York Academy of Medicine 42, 65-78 (1966).

(10) Garth Boehm, L.Y., Liang Han, Qiang Zheng. Development of the generic drug industry in the US after the Hatch-Waxman Act of 1984 Acta Pharmaceutica Sinica B 3, 297-311 (2013).

(11) Rumore, M.M. The Hatch-Waxman Act--25 Years Later: Keeping the Pharmaceutical Scales Balanced. <http://www.pharmacytimes.com/publications/supplement/2009/genericsupplement0809/generichatchwaxman-0809> (2009). Accessed April 12016.

(12) S.1538 - An act to amend the Federal Food, Drug, and Cosmetic Act to revise the procedures for new drug applications, to amend title 35, United States Code, to authorize the extension of the patents for certain regulated products, and for other purposes. (U.S. Congress, 1984).

(13) (2013). Declining medicine use and costs: for better or worse? A review of the use of medicines in the United States in 2012. 56 (IMS institute for healthcare infomatics, USA, 2013).

(14) Facts and figures 2014: on pharmaceutical care in The Netherlands. <https://www.sfk.nl/english/ Dataenfeiten2014_A4_magazine_web.pdf> (2014).

(15) Kmietowicz, Z. Company managed to block development of generic drug. Bmj 336, 576 (2008).

(16) Arnum, P.V. The changing business models for innvator-drug and generic-drug companies. <http://www.pharmtech.com/changing-business-models-innovator-drug-and-generic-drug-companies> (2010). Accessed June 092016.

(17) Toverud, E.L., Hartmann, K. \& Hakonsen, H. A Systematic Review of Physicians' and Pharmacists' Perspectives on Generic Drug Use: What are the Global Challenges? Applied health economics and health policy 13 Suppl 1, S35-45 (2015).

(18) Privitera, M.D. Generic antiepileptic drugs: current controversies and future directions. Epilepsy Curr 8, 113-7 (2008).

(19) Meredith, P.A. Potential concerns about generic substitution: bioequivalence versus therapeutic equivalence of different amlodipine salt forms. Curr Med Res Opin 25, 2179-89 (2009).

(20) Crawford, P., Hall, W.W., Chappell, B., Collings, J. \& Stewart, A. Generic prescribing for epilepsy. Is it safe? Seizure 5, 1-5 (1996).

(21) Borgheini, G. The bioequivalence and therapeutic efficacy of generic versus brand-name psychoactive drugs. Clin Ther 25, 1578-92 (2003).

(22) Atif, M., Azeem, M. \& Sarwar, M.R. Potential problems and recommendations regarding substitution of generic antiepileptic drugs: a systematic review of literature. SpringerPlus 5, 182 (2016). 


\section{Chapter 1}

(23) Wilner, A.N. Therapeutic equivalency of generic antiepileptic drugs: results of a survey. Epilepsy Behav 5, 995-8 (2004).

(24) Zachry, W.M., 3rd, Doan, Q.D., Clewell, J.D. \& Smith, B.J. Case-control analysis of ambulance, emergency room, or inpatient hospital events for epilepsy and antiepileptic drug formulation changes. Epilepsia 50, 493-500 (2009).

(25) Kesselheim, A.S. et al. Seizure outcomes following the use of generic versus brand-name antiepileptic drugs: a systematic review and meta-analysis. Drugs 70, 605-21 (2010).

(26) Oluboka, O., Stewart, S., Landry, S. \& Adams, S. Does therapeutic equivalence follow bioequivalence? A randomized trial to assess clinical effects after switching from Clozaril to generic clozapine (genclozapine). Journal of clinical pharmacology 50, 531-5 (2010).

(27) Kesselheim, A.S. et al. Clinical equivalence of generic and brand-name drugs used in cardiovascular disease: a systematic review and meta-analysis. JAMA : the journal of the American Medical Association 300, 2514-26 (2008).

(28) Chen, M.L., Patnaik, R., Hauck, W.W., Schuirmann, D.J., Hyslop, T. \& Williams, R. An individual bioequivalence criterion: regulatory considerations. Statistics in medicine 19, 2821-42 (2000).

(29) Midha, K.K., Rawson, M.J. \& Hubbard, J.W. Bioequivalence: switchability and scaling. European journal of pharmaceutical sciences : official journal of the European Federation for Pharmaceutical Sciences 6, 8791 (1998).

(30) Kesselheim, A.S. et al. Variations in pill appearance of antiepileptic drugs and the risk of nonadherence. JAMA internal medicine 173, 202-8 (2013).

(31) Question \& Answers: Positions on specific questions addressed to the pharmacokinetics working party. (Committee for Medicinal Products for Human use (CHMP), European Medicine Agency, London, 2014).

(32) Committee for Proprietary Medicinal Products Working Party in the Efficacy of Medicinal Products: The Rule Governing Medicinal Products in the European Community (1991).

(33) Note for Guidance on the Investigation of Bioavailability and Bioequivalence. (Committee for Medicinal Products for Human use (CHMP), European Medicine Agency, London, 2001).

(34) Lionberger, R.A. FDA critical path initiatives: opportunities for generic drug development. The AAPS journal 10, 103-9 (2008).

(35) Maliepaard, M., Yu, Y. \& Leufkens, H. Equivalence of generic medicines in general and immunosuppressants in particular - a regulatory opinion on switching of ciclosporin, tacrolimus and mycophenolate mofetil. Generics and Biosimilars Initiative Journal 2, 86-90 (2013).

(36) Peterson, G.M. Generic Substitution of Antiepileptics: Need for a Balanced View. CNS Spectr, (2011).

(37) Garcia-Arieta, A. \& Gordon, J. Bioequivalence requirements in the European Union: critical discussion. The AAPS journal 14, 738-48 (2012).

(38) In Vivo bioequivalence studies based on population and individual bioequivalence approaches. . (ed. U.S. Department of Health and Human Services, F.a.D.A., Center for Drug Evaluation and Research (CDER)) (1997).

(39) Hauck, W.W. \& Anderson, S. Measuring switchability and prescribability: when is average bioequivalence sufficient? Journal of pharmacokinetics and biopharmaceutics 22, 551-64 (1994).

(40) Guidance for industry: Average, population, and individual approaches to establishing bioequivalence (ed. U.S. Department of Health and Human Services, F.a.D.A., Center for Drug Evaluation and Research (CDER)) (1999).

(41) Chow, S.-C. Individual bioequivalence-A review of FDA draft guidance. Drug Information Journal 33, 10 (1999).

(42) Anderson, S. \& Hauck, W.W. The transitivity of bioequivalence testing: potential for drift. Int J Clin Pharmacol Ther 34, 369-74 (1996).

(43) Maliepaard, M., Banishki, N., Gispen-de Wied, C.C., Teerenstra, S. \& Elferink, A.J. Interchangeability of generic anti-epileptic drugs: a quantitative analysis of topiramate and gabapentin. Eur J Clin Pharmacol 67, 1007-16 (2011). 
(44) Gwaza, L. et al. Statistical approaches to indirectly compare bioequivalence between generics: a comparison of methodologies employing artemether/lumefantrine 20/120 mg tablets as prequalified by WHO. Eur J Clin Pharmacol 68, 1611-8 (2012).

(45) Christians, U. Generic immunosuppressants: the European perspective. Transplantation proceedings 31, 19S-22S (1999).

(46) Amidon, K.S., Langguth, P., Lennernas, H., Yu, L. \& Amidon, G.L. Bioequivalence of oral products and the biopharmaceutics classification system: science, regulation, and public policy. Clin Pharmacol Ther 90 , 467-70 (2011).

(47) Davit, B.M. et al. Implementation of a reference-scaled average bioequivalence approach for highly variable generic drug products by the US Food and Drug Administration. The AAPS journal 14, 915-24 (2012).

(48) Product-Specific Recommendations for Generic Drug Development. <http://www.fda.gov/Drugs/ GuidanceComplianceRegulatoryInformation/Guidances/ucm075207.htm>. Accessed 17-04 2016.

(49) Product-specific bioequivalence guidance. <http://www.ema.europa.eu/ema/index.jsp?curl=pages/ regulation/general/general_content_000625.jsp\&mid=WC0b01ac0580848f74>. Accessed 17-04 2016. 

Chapter

\section{Equivalence of generic medicines in general and immunosuppressants in particular - a regulatory opinion on switching of ciclosporin, tacrolimus and mycophenolate mofetil}

Marc Maliepaard ${ }^{1}$, Yang Yu ${ }^{1,2}$, Hubert G.M. Leufkens ${ }^{1,3}$

${ }^{1}$ Dutch Medicines Evaluation Board, Utrecht, The Netherlands

${ }^{2}$ Department of Pharmacology, Maastricht University Medical Centre, Maastricht, The Netherlands

${ }^{3}$ Utrecht Institute for Pharmaceutical Sciences, Division of Pharmacoepidemiology and Clinical Pharmacology, Utrecht University, Utrecht, The Netherlands

Generics and Biosimilars Initiative Journal (GaBI Journal). 2013;2(2):86-90.

This paper is based on and expended from a pro-con discussion that appeared in the Nederlands Tijdschrift voor Nefrologie (Journal of the Dutch Renal Transplant Society) in February 2012. 


\section{ABSTRACT}

This position paper deals with our regulatory opinion on registered generic immunosuppressants such as ciclosporin, tacrolimus and mycophenolate mofetil, and provides arguments why these medicines are considered equally safe and effective as the branded drug based on demonstrated bioequivalence. Though regulators acknowledge the worries from the field, we are of the opinion that there are no compelling pharmacological arguments to date against the sensible use of generic immunosuppressants in clinical practice, under the shared and mutual care by prescribers and pharmacists.

Keywords: bioequivalence, drug safety, generic, immunosuppression, regulation 


\section{BACKGROUND}

As soon as the protection period of 10 years of a branded drug has expired, it is possible to seek a marketing authorization for a generic form of this drug. This gives rise to the situation that patients may no longer be treated with the original product (proprietary, branded drug), but with a generic medicine. In that case generic substitution takes place, where the branded drug is exchanged with a product with an identical active ingredient. In the last 30 years we have gained extensive experience with such generics substitution, not only in The Netherlands but also in other parts of Europe and the US. For example, in The Netherlands, treatment with generics has now become the standard for drugs such as statins, proton pump inhibitors and antihypertensive drugs.

Though the Dutch Medicines Evaluation Board (College ter Beoordeling van Geneesmiddelen, MEB) is not directly involved in the actual substitution strategy in The Netherlands, registration of generic medicines will only take place if the MEB is convinced that the generic medicine has the same efficacy and safety profile as the innovator medicine. As our contribution to the discussion on generics substitution, we explain why the MEB considers this to be the case also for immunosuppressants, based on the quality of the medicine and bioequivalence testing.

\section{GENERIC FACTS}

What are the facts on generic medicines? A generic medicine is a product with the same active ingredient, the same strength and the same pharmaceutical form as the branded drug (in other words, is pharmaceutically equivalent). If the manufacturer of the generic drug product demonstrates that its exposure in time (which, for products with immediate release characteristics, is determined by area under the curve $(A \cup C)$ and $C_{\max }$ ) is equal to that of the branded medicine - so the two products are bioequivalent - the generic and branded medicines are considered to be therapeutically equivalent. This assumption is logical, because when a drug is absorbed in the same way (as demonstrated by the bioequivalence study), its further pharmacological behavior only depends on the characteristics of the molecular active ingredient. The potential differences in inactive excipients between branded and generic drug formulation are then no longer relevant. For generic and branded drugs, the molecular active substance is qualitatively and quantitatively the same. Therefore, once bioequivalence is demonstrated, the company that manufactures the generic drug can refer to the clinical studies performed with the branded drug for the efficacy and safety of the generic drug product, with no need for additional clinical trials prior to registration.

In most cases, bioequivalence is demonstrated in healthy volunteers (1). It is well known that the exposure in healthy volunteers may be different than that in patients, due to comorbidities of the patient. However, it is important to realize that this will 
affect branded and generic medicines equally. In addition, the actual exposure in a healthy volunteer is the result of a combination of endogenous factors, including renal and hepatic function, metabolizer status, e.g. poor or extensive metaboliser; ethnic background, and gastric $\mathrm{pH}$, which affect a drug's absorption, metabolism and elimination. When comparable exposure between a branded and generic medicine has been demonstrated in a healthy volunteer, relative exposure in patients, determined by a different mix of these endogenous characteristics, also is expected to yield comparable exposure. Versantvoort et al.(2) illustrated this principle with a bioequivalence study in which a poor metabolizer was present among extensive metabolizers: though the exposure in the poor metabolizer was dramatically higher - probably even requiring a dose adjustment in clinical practice - the relative exposure of the branded and generic drug within this subject remained comparable. The same principle will hold for other comorbidities, like renal or hepatic impairment: when bioequivalence has been demonstrated in healthy volunteers, the relative exposure change will be the same for the branded and generic medicine. Therefore, bioequivalence demonstrated in healthy volunteers will be valid for the patient population.

In most cases, excipients are inactive, and a single-dose bioequivalence study is considered sufficient to obtain registration of a generic drug. If active excipients, such as in a gastro-resistant coating, are present in the drug formulation, additional data specifically relevant to this active excipient are required in order to demonstrate that the excipient's behavior is comparable with that of the branded medicine. For example, in case of a gastro-resistant coating, comparable $\mathrm{pH}$ dependent dissolution should be demonstrated, and an additional bioequivalence study with food (resulting in increased gastric $\mathrm{pH}$ ) should be provided (3). For other specific formulations, e.g. liposomal, sorbitol, cyclodextrin or microemulsion containing formulations, other specific additional requirements are needed $(3,4)$.

Of note, bioequivalence studies are not only used for registration of generic medicines, but also in drug development of a newly invented medicine where appropriate (5), or a line extension after registration of a branded medicine. For instance, registration of the $0.5 \mathrm{mg}$ Prograf ${ }^{\circ}$ strength was based on a bioequivalence study under singledose conditions (6). It is therefore clear that identical regulations are used both for branded and generic products, and thus, these medicines undergo the same rigorous scrutiny upon admission.

Normally, bioequivalence is considered to have been demonstrated when the $90 \%$ confidence intervals of the generic:branded ratios for AUC as well as $C_{\max }$ are within 80$125 \%$. These acceptance criteria are strict, and are outlined in the Guideline on the Investigation of Bioequivalence (1). Additional stringent requirements are placed on the actual analytical assay that is used in such bioequivalence studies to quantify the plasma or blood concentrations (7). With respect to generic immunosuppressants, additional care has been taken by the regulatory authorities, by narrowing the acceptance range for some immunosuppressants, in order to further reduce the likelihood of obtaining 
clinically relevant differences in exposure when switching to and from generic medicines. The option to narrow the acceptance range is given in the current (2010) as well as the previous version (2001) of the Guideline on the Investigation of Bioequivalence, for medicines with a narrow therapeutic index (NTI). Since a worldwide definition of an NTI is lacking, this is considered by the European Medicines Agency (EMA) on a case-bycase basis. Indeed, the acceptance criteria for generic immunosuppressants have been adjusted, i.e., to $90-111 \%$ for ciclosporin $A \cup C$ and $C_{\max }$, and to $90-111 \%$ and $80-125 \%$ for tacrolimus $A U C$ and $C_{\text {max }}$, respectively (3). Due to the microemulsion formulation applied in Neoral, which has led to a pronounced increase in predictability of the ciclosporin exposure and reduced food effect as compared to its precursor Sandimmune, bioequivalence for ciclosporin generics should be demonstrated under fasted as well as fed conditions. With regard to tacrolimus, only the $90 \%$ confidence intervals for AUC was narrowed, since due to accumulation of tacrolimus upon repeated dosing, a potential difference between formulations in $\mathrm{C}_{\max }$ after single dosing can be expected to be less at steady state, if AUC is the same for the two formulations. Therefore, the normal acceptance criteria for $\mathrm{C}_{\max }$ can be used in single-dose bioequivalence studies for tacrolimus (3). For mycophenolate mofetil, for which bioequivalence is demonstrated based on exposure of the mycophenolic acid metabolite, no narrowing of the criteria was considered necessary by the EMA (3).

Overall, the strict requirements for demonstrating bioequivalence are equally valid for branded and generic drug products. Thus, the demonstration of bioequivalence is strong evidence to secure the substitution of a generic product for the branded medicine.

\section{GENERIC DOUBTS}

Nevertheless, questions arise from a number of clinical disciplines that, due to claimed specific characteristics within their patient population, some patients are not suitable for generic substitution. One of these disciplines is transplantation medicine. Concerns regarding the substitution of immunosuppressants by generic drug products are understandable from the recipient's perspective: the impact of failing immunosuppressant therapy following transplantation can be dramatic. In the scientific literature, some publications support generic substitution, e.g. by suggesting comparable efficacy and safety with ciclosporin generic formulations as with branded equivalents (8-12). Similar support comes from demonstrations of bioequivalence of generic and branded tacrolimus in kidney transplant patients (13) and comparable clinical outcomes with branded or generic tacrolimus in kidney and liver transplant patients $(14,15)$, with the routinely applied therapeutic dose monitoring for tacrolimus being advised as a safeguard (16, 17). Conversely, over the past few years, a number of reviews and clinical guidelines raise concerns about generics substitution $(18,19)$. In Europe, the European Society for 
Organ Transplantation (ESOT) published recommendations on generics substitution of immunosuppressive drugs, which were based on the guideline drafted by the Dutch Renal Transplant Society (18). Although some concerns in the ESOT recommendations are acknowledged, overall, in our opinion there appears to be an overemphasis on assumed shortages of pharmacokinetic (PK) or clinical data relating to generic drug product registration. Examples of such assumptions include those relating to the $C_{\text {trough }} / C_{\text {min }}$, multiple dose conditions, or the fact that bioequivalence between different generics is not formally tested. These topics are discussed below. Although the requirements posed by the regulatory authorities on the description of PK of generics are limited (i.e., almost equal $A \cup C$ and $C_{\max }$, and similar quality), these requirements are well thought over. Many of the concerns raised regarding generics substitution are not deducible to scientific facts or studies, but often involve a number of recurring arguments which are demonstrably incorrect. We discuss a number of those related to immunosuppressants below.

One incorrect assumption often expressed is that, though AUC and $C_{\max }$ obtained with a branded immunosuppressive drug and its generic may be the same, there may still be differences in certain critical points of the plasma concentration-time curves. This argument has been expressed for ciclosporin, where plasma concentrations two hours after administration $\left(C_{2}\right)$ or trough levels $\left(C_{\text {trough }}\right)$ are used to monitor and adjust ciclosporin exposure and dose (18). However, for an immediate-release product like ciclosporin the PK after the initial absorption from the gastrointestinal tract is essentially governed by the molecular active substance only. Since this substance is identical for the branded and generic ciclosporin formulations, differences in $C_{2}$ or $C_{\text {trough, despite }}$ comparable $A \cup C$ and $C_{\max }$ in the case of demonstrated bioequivalence, will be an extremely unlikely event. In a field that is so familiar with therapeutic drug monitoring, it is remarkable that this is seen as a possibility.

Another argument raised is that bioequivalence for immunosuppressants should be demonstrated under steady-state conditions instead of the currently required singledose conditions only, since in clinical practice steady-state conditions may be more important (18). It is agreed that in clinical practice steady-state conditions are important, and it is acknowledged that for certain medicinal products the absolute exposure under steady-state may be different from that after a single dose, due to accumulation upon multiple dose administration. However, there is no reason to assume that the relative exposure obtained under single-dose conditions will be different from that under steady-state conditions. It is well known that the sensitivity of detecting a difference in exposure between two different formulations under steady-state conditions is less than after a single dose (1). Viewed from the opposite perspective, assessment of bioequivalence under steady-state conditions for ciclosporin would lead to a less stringent assessment of bioequivalence. Applying lower standards for generics is certainly not acceptable to authorities as the Dutch MEB and EMA. After absorption of a medicinal product, its PK is only determined by the molecular active substance. Therefore, 
there is no reason to assume that the PK behavior will be different for an immediaterelease generic drug product compared with the branded drug under steady-state conditions, when a comparable absorption has been demonstrated under the most sensitive condition, i.e., after single dose administration.

In certain cases, therapeutic substitution (the exchange of two different types of formulations or two different active ingredients for the same indication) appears to be used to indicate that presumed problems with generic substitution are plausible (18). This is exemplified by the reported reference to the product description (Summary of Product Characteristics, SmPC) of tacrolimus formulations, which contain a warning that patients must remain on the same formulation. This warning makes sense, and it is clear that the underlying reason for this warning is the fact that there are different types of branded tacrolimus formulations with different release characteristics and therefore different pharmacokinetics on the market, namely Prograf, being an immediate release formulation given twice daily and Advagraf, a prolonged release formulation for once daily administration. Everyone would agree that these different formulations, which are intended for either once daily or BID (twice a day) dosing, should not be interchanged, and indeed issues upon accidentally interchanging these two branded tacrolimus formulations have been reported. However, it is unjust to extrapolate founded warnings in the tacrolimus SmPC against substitution between different types of tacrolimus formulations to substitution between equivalent types of tacrolimus formulations, as in the case of generics substitution, where the release characteristics are equivalent.

The suspicion that generic-generic substitution leads to increased, potentially clinically relevant variability in exposure, which is also used as an argument against generics substitution (18), has not been demonstrated. The occurrence of greater, possibly clinically significant, differences in exposure is a theoretical possibility, which would occur when $90 \%$ confidence intervals of different generics would be in the opposite part of the $80-125 \%$ criterion. However, given the small observed difference in mean exposure between an arbitrary generic and branded drug (20), the occurrence of great differences in exposure upon generic-generic substitution seems unlikely, though formally it cannot be excluded. For the antiepileptic drugs gabapentin and topiramate, which are registered in The Netherlands, the absence of increased differences in exposure when different generics were exchanged was shown by research conducted at the MEB using bioequivalence data obtained from registration files at the MEB. These data were used to estimate $90 \%$ confidence intervals following the substitution of different generic formulations of gabapentin or topiramate (21). Research towards such simulated generic-generic substitution data for immunosuppressants is currently ongoing at the MEB. In that respect, it is important to note that ciclosporin generics in The Netherlands were registered prior to the narrowed acceptance criteria of $90-111 \%$ for this product, both under fasting and fed conditions, implemented by EMA. MEB closely monitors any signs of unacceptable efficacy or safety reports related to these drugs. 
Despite the arguments provided above, it cannot be disputed that in certain isolated cases, issues with generics are reported. However, these are considered as exceptional cases, e.g. sometimes related to intolerance to certain excipients like lactose, fructose or galactose, which may be present in generics and not in branded products (and vice versa). However, in the vast majority of switches, substitution proceeds without problems. It is acknowledged that factors other than differences in exposure may play a role in the perception of generics and the outcome of generics substitution in patients, for example, with differences in shape and colour of generics, which may lead to distrust, mistakes or reduced compliance among patients. The consequences of such differences may even increase when the branded and generic drug are frequently changed, which is a realistic scenario in The Netherlands, where the frequency of switching has increased over the years due to the current pricing and reimbursement policy of the Dutch health insurance companies. Frequent switching to other generics may be expected to negatively affect compliance and confidence, could potentially increase the chance of errors, and should therefore be avoided as much as possible.

It is the joint responsibility of the pharmacist and prescriber to monitor this switching and to provide satisfactory communication for the benefit of the patient, in case generic substitution takes place. In our opinion, inadequate communication between pharmacist and prescriber cannot be used as an argument against the use of generics (18), but should better lead to incentives to solve this issue.

Uncertainty about the underlying principles and legislation of generics, combined with otherwise well appreciated and valued patient care, appear to be leading in the frequently provided arguments against generics substitution, rather than solid evidence for the occurrence of problems. It is reasonable to assume that a well-informed prescriber is able to play a major role in the perception of generic immunosuppressants by the patient, and in that respect, MEB should also take a part in this discussion and education.

Regulatory agencies like MEB are actively involved in governing the safe use of generic immunosuppressants. Pharmacovigilance structures are in place, and adverse events reported related to immunosuppressants, as well as other medicinal products, are taken very seriously. In case there are signs of unexpected disproportional adverse events or inefficacy with any drug - be it a generic or branded - MEB is obliged to take action. For the pharmacovigilance system to work, it is essential to report issues to the relevant pharmacovigilance centres in the different EU Member States, in order to be able to keep a close eye on the actual quality of generics, and to reduce the time before a signal can be picked up. In that sense, regulations have recently been amended with a more pronounced place for reporting adverse events by patients, who are considered 'hands-on' experts. 


\section{CONCLUSIONS}

From a regulatory point of view, generic immunosuppressants like ciclosporin, tacrolimus and mycophenolate mofetil are considered as safe and effective as the branded drug based on demonstrated bioequivalence, and therefore considered interchangeable. Though we are aware of worries expressed in the field, we are of the opinion that there are no compelling pharmacological arguments to date against the sensible use of generic immunosuppressants in clinical practice, under the shared and mutual care of prescribers and pharmacists.

\section{FOR PATIENTS}

Generic drugs are prescribed more and more. Sometimes, the change of prescription from branded to a generic medicine leads to unrest and doubts among patients, e.g. on whether generic drugs are equally safe and work equally well as the branded medicines. These doubts are acknowledged and understood. In this paper, we aim to clarify what is done by regulators to safeguard the use of generics as much as possible. From the prescriber's and pharmacist's perspectives, we expect and promote a professional and adequate collaboration to take appropriate action in isolated cases when a generic drug does not meet its expectation in an individual patient. 


\section{REFERENCES}

(1) Guideline on the investigation of bioequivalence http://www.ema.europa.eu/docs/en_GB/document_ library/Scientific_guideline/2010/01/WC500070039.pdf accessed 19-03-2013

(2) Versantvoort C, Maliepaard M, Lekkerkerker F. Generics: what is the role of registration authorities. Neth J Med. 2008;66:62-66.

(3) Questions \& Answers: Positions on specific questions addressed to the Pharmacokinetics working party. http://www.ema.europa.eu/docs/en_GB/document_library/Scientific_guideline/2009/09/WC50000296 3.pdf, accessed 19-03-2013

(4) Reflection paper on the data requirements for intravenous liposomal products developed with reference to an innovator liposomal product. http://www.ema.europa.eu/docs/en_GB/document_library/Scientific_ guideline/2013/03/WC500140351.pdf. accessed 19-03-2013

(5) Baumgärtel C. Myths, questions, facts about generic drugs in the EU. GaBI Journal 2012;1:34-48. DOI: 10.5639/gabij.2012.0101.009

(6) Bekersky I, Dressler D, Boswell GW, Fergen B, Tracewell W, Mekki Q. Bioequivalence of a new strength tacrolimus capsule under development. Transplant Proc 1998;30:1457-1459.

(7) Guideline on bioanalytical method validation http://www.ema.europa.eu/docs/en_GB/document_ library/Scientific_guideline/2011/08/WC500109686.pdf, accessed 19-03-2013

(8) Kahn D, Muller E, Pascoe M. Safe conversion to cicloral, a generic cylosporine, in both stable and de novo renal transplant recipients. Saudi J Kidney Dis Transpl. 2010;21:426-432.

(9) Diarra DA, Riegersperger M, Säemann MD, Sunder-Plassmann G. Maintenance immunosuppressive therapy and generic cyclosporine A use in adult renal transplantation: a single center analysis. Kidney Int Suppl. 2010;115:S8-S11.

(10) Tarek BO, Nadia BF, Anis K, Saloua L, Abderrahmane A, Lamia T, Amel L, Mohamed L. Assessment of bioequivalence of a generic cyclosporine (Equoral) by a prospective randomized controlled trial on allogeneic stem cell transplant recipients. Transplant Proc. 2010;42:3735-8.

(11) Niemczyk M, Paczek L. Generic formulation of Cyclosporine A, Equoral ${ }^{\circledR}$, in de novo kidney transplant recipients: five-year follow-up. Ann Transplant. 2011;16:59-62.

(12) Durlik M, Paczek L, Rutkowski B, Lewandowska D, Debska-Slizien A, Chamienia A, Wyzgal J, OgnistaGajda A, Niemczyk M. The efficacy and safety of ciclosporin (Equoral ${ }^{\circledR}$ ) capsules after renal transplantation: A multicentre, open-label, phase IV clinical trial. Ann Transplant. 2010;15:51-59.

(13) Alloway RR, Sadaka B, Trofe-Clark J, Wiland A, Bloom RD. A randomized pharmacokinetic study of generic tacrolimus versus reference tacrolimus in kidney transplant recipients. Am J Transplant. 2012;12:2825-31.

(14) Spence MM, Nguyen LM, Hui RL, Chan J. Evaluation of Clinical and Safety Outcomes Associated with Conversion from Brand-Name to Generic Tacrolimus in Transplant Recipients Enrolled in an Integrated Health Care System. Pharmacotherapy. 2012;31:1130. [Epub ahead of print]

(15) Ensor CR, Trofe-Clark J, Gabardi S, McDevitt-Potter LM, Shullo MA. Generic maintenance immunosuppression in solid organ transplant recipients. Pharmacotherapy. 2011;31:1111-1129. Review.

(16) McDevitt-Potter LM, Sadaka B, Tichy EM, Rogers CC, Gabardi S. A multicenter experience with generic tacrolimus conversion. Transplantation. 2011;92:653-657.

(17) Momper JD, Ridenour TA, Schonder KS, Shapiro R, Humar A, Venkataramanan R. The impact of conversion from prograf to generic tacrolimus in liver and kidney transplant recipients with stable graft function. Am J Transplant. 2011;11:1861-1867.

(18) van Gelder T. Advisory Committee recommendations on generic substitution of immunosuppressive drugs. Transplant International 2011;24:1135-1141.

(19) Harrison JJ, Schiff JR, Coursol CJ, Daley CJ, Dipchand Al, Heywood NM, Keough-Ryan TM, Keown PA, Levy GA, Lien DC, Wichart JR, Cantarovich M. Generic immunosuppression in solid organ transplantation: a Canadian perspective. Transplantation. 2012;93:657-665. 
(20) Davit BM, Nwakama PE, Buehler GJ, Conner DP, Haidar SH, Patel DT, Yang Y, Yu LX, Woodcock J. Comparing generic and innovator drugs: a review of 12 years of bioequivalence data from the United States Food and Drug Administration. Ann Pharmacother 2009;43:1583-1597.

(21) Maliepaard M, Banishki N, Gispen-de Wied CC, Teerenstra S, Elferink AJ. Interchangeability of generic anti-epileptic drugs: a quantitative analysis of topiramate and gabapentin. Eur J Clin Pharmacol 2011;67:1007-1016. 



\section{Chapter}

\section{A comparison of the intrasubject variation of drug exposure between generic and brand-name drugs: a retrospective analysis of replicate design trials}

$\underline{\text { Yang } \mathrm{Yu}^{1,2}}$, Steven Teerenstra ${ }^{2,3}$, Cees Neef ${ }^{1,4}$, David Burger ${ }^{5}$ and Marc Maliepaard ${ }^{2}$

${ }^{1}$ Dept. Pharmacology and Toxicology, CARIM, Maastricht University Medical Centre, Maastricht, The Netherlands

${ }^{2}$ Medicines Evaluation Board, Utrecht, The Netherlands

${ }^{3}$ Dept. for Health Evidence, section Biostatistics, Radboud university medical centre, Nijmegen, The Netherlands

${ }^{4}$ Dept. Pharmacology and Toxicology, CAPHRI, Maastricht University Medical Centre, Maastricht, The Netherlands

${ }^{5}$ Dept. of Pharmacy, Radboud University Nijmegen Medical Centre, Nijmegen, The Netherlands

British Journal of Clinical Pharmacology 81(4), 667-78 (2016) 


\section{ABSTRACT}

Aim(s) To investigate whether differences in total and peak drug exposure upon generic substitution are due to differences between formulations or to intrasubject pharmacokinetic variability of the active substance.

Methods This study was designed as a retrospective reanalysis of existing studies. Nine replicate design bioequivalence studies representing six drug classes were retrieved from Dutch regulatory authority, i.e. for alendronate, atorvastatin, cyclosporin, ebastine, exemestane, mycophenolate mofetil, and ropinirole.

Results In most studies the intrasubject variability in total and peak drug exposure was comparable for the brand-name (in the range of 0.01-0.24 for $A \cup C_{t}$ and 0.02-0.29 for $C_{\max }$ on a log-scale) and generic drugs (0.01-0.23 for $A \cup C_{t}$ and 0.08-0.33 for $C_{\max }$ ), and was comparable with the intrasubject variability upon switching between those drugs (0.01-0.23 for $A \cup C_{t}$ and 0.06-0.33 for $C_{\max }$ ). The variance related to subject-byformulation interaction can be considered negligible (-0.069-0.047 for $A \cup C_{t}$ and $-0.091-$ 0.02 for $\left.C_{\max }\right)$.

Conclusion In the investigated studies, the variation in total and peak exposure seen when a patient is switched from a brand-name drug to a generic drug is comparable to the variation seen following repeated administration of the brand-name drug in that patient. Only the intrasubject variability seems to play a crucial and decisive role in the variation in drug exposure seen; no additional formulation dependent variation in exposure is observed upon switching. Thus, our data support that, from a clinical pharmacological perspective, the benefit-risk of a generic drug is comparable to that of the brandname drug for the medicines that were included in this investigation.

Key words: intrasubject variability, generic drugs, bioequivalence study 


\section{INTRODUCTION}

Worldwide, the $80-125 \%$ criterion is used as the standard limit for the $90 \%$ confidence intervals (Cls) of the In-transformed ratios between generic and brand-name drugs for the area under the drug concentration-time curve $\left(A \cup C_{t}\right)$ and the maximum plasma concentration $\left(\mathrm{C}_{\max }\right)$ in bioequivalence studies, and meeting this criterion has been the basic requirement for registration of a generic drug for more than 25 years $(1,2)$, known as average bioequivalence approach. Thus a small difference in average total or peak exposure between a generic drug and a brand-name drug is permissible and is considered not to have clinical consequences. However, bioequivalence between a generic drug and a brand-name drug does not exclude the possibility that differences in the exposure occur at an individual level, due to e.g. intrasubject variability and the variance due to subject-by-formulation effects. Thus, in principle, it is possible that there is a detectable difference in plasma drug levels after an individual patient switches from a brand-name drug to a generic drug and wise versa. Indeed, a difference in exposure upon switching (either decreased or increased exposure) has been reported $(3,4)$. This difference is partly responsible for concerns among users and prescribers about the interchangeability of generic drugs. Some reports suggest that brand-name and generic drugs may not be equally effective or safe because they may be associated with a difference in exposure in individual patients. For example, an increased risk of seizures, frequent visits to the emergency unit, and a high switching-back rate have been reported when patients with epilepsy were switched from a brand-name drug to a generic drug (4-9). Yet a systematic review and meta-analysis of clinical studies of switching between antiepileptic drugs found no difference in the odds of uncontrolled seizures in patients treated with generic versus brand-name drugs(10). A similar clinical equivalence of generic and brand-name drug has been reported for drugs for cardiovascular diseases (11).

Because of the fact that the traditional bioequivalence study based on the average bioequivalence approach cannot evaluate the differences in drug exposure levels in terms of $A \cup C_{t}$ and $C_{\max }$ in individuals, population bioequivalence and individual bioequivalence approaches have been proposed for assessing the total variability of $A \cup C_{t}$ and $C_{\max }$ in the population, and intrasubject variability for the generic and brand-name drugs and the subject-by-formulation interaction, respectively(12, 13). In 1997, U.S. Food and Drug Administration (FDA) drafted guidelines on the investigation of bioequivalence based on population and individual bioequivalence approaches(14, 15), aiming to resolve the concerns on prescribability and switchability in individual patients. However, the use of these alternative approaches was not successful due to the issues in masking effect, power and sample size determination, statistical procedures and study design (16). In the literature, it has been shown that the proposed criteria using a mixed-scaling aggregate strategy would lead to a relaxation of the $80-125 \%$ average bioequivalence standard, particularly for highly variable drugs (17). A positive correla- 
tion between the intrasubject variability and the estimation of the variance for subjectby-formulation interaction was identified, which consequently eliminated the possibility of using a constant level (such as 0.0225 suggested by FDA) as a basis for demonstrating substantial subject-by-formulation interactions (18).

In the literature, the reported individual differences in exposure between brandname and generic drugs have been postulated to be related to individual-specific variability in pharmacokinetics $(19,20)$, which leads to a variation in drug exposure in individuals. Given that the human body is a dynamic environment when it comes to pharmacokinetics, it is perhaps not surprising that drug absorption and excretion may vary from time to time, resulting in variable drug exposure from dose to dose, either with repeated administration of the same drug, or when switching between brand-name and generic drugs. However, to our knowledge, it has not yet been studied whether intrasubject variability is a key factor in the altered total or peak drug exposure when a patient is switched to a generic drug or to what extent the intrasubject variability contributes to the variation observed when switching to a generic drug.

The aim of this investigation was to investigate the reason for the difference in total and peak drug exposure in individuals that is observed when switching between a brand-name drug and a generic drug, and to clarify the role of intrasubject variability in pharmacokinetics in this. To our knowledge, the role of intrasubject variability in explaining differences in exposure obtained upon switching between brand-name and generic drugs has not yet been fully clarified. For this purpose, we made use of data obtained from replicate design studies. Typically, these are four-way crossover study in which each subject randomly receives, under fasting conditions, the brand-name drug twice and the generic drug twice. This replicate study design makes it possible to investigate the intrasubject variability in pharmacokinetic parameters (i.e., total and peak drug exposure) with the brand-name drug, the generic drug, and the situation of switching between the two drugs. It should be noted that in the latter case, the intrasubject variability includes the effect of difference in formulations (the brand-name vs. generic) and other unknown factors caused by study design or trial handling. In addition, the variance related to the subject-by-formulation interaction may be critical for demonstration of bioequivalence in individuals. In our investigation, replicate design studies were used to study whether differences in exposure between brand-name and generic drugs are actually due to intrasubject variability in exposure in an individual and a population. We also investigated whether pharmacokinetic parameters (i.e., $A \cup C_{t}$ and $C_{\max }$ ) measured after repeated administration of a brand-name drug $\left(R_{2}: R_{1}\right)$ or a generic drug $\left(T_{2}: T_{1}\right)$ or upon switching ( $\left.R: T\right)$ differ from individual to individual (i.e. due to the variance related to subject-by-formulation interaction). By doing so, we aimed to obtain insight into the potential difference in drug exposure that can be seen when a patient switches between brand-name and generic drugs. 


\section{METHODS}

We retrieved nine replicate design bioequivalence studies from the Dutch regulatory authority's database in June 2013. The use of these data has been approved for publication by the Dutch Medicine Evaluation Board (MEB). These studies had been submitted to the authority in support of the registration of generic drugs, and most of these generic drugs are currently available in the Netherlands and some other European countries. Basic information about these studies and study outcome is provided in Table 1. These studies investigated seven different active substances, namely, alendronate, atorvastatin, cyclosporin, ebastine, exemestane, mycophenolate mofetil, and ropinirole, which were from six drug classes (i.e. drug for treatment of bone diseases, lipid modifying agents, immunosuppressants, antihistamines for systemic use, endocrine therapy and anti-Parkinson drugs). With the exception of the study on ebastine, which had a three-way crossover, partial replicate design study (in a sequence of R-R-T, R-T-R or T-R$R)$, all studies were designed as a four-way crossover replicate study, in which each subject received two single treatments with the brand-name drug $\left(R_{1}\right.$ and $\left.R_{2}\right)$ and two treatments with the generic drug $\left(T_{1}\right.$ and $\left.T_{2}\right)$ in a sequence of R-T-R-T or T-R-T-R. No carry-over effects were identified in all studies.

Total and peak drug exposure were investigated in terms of the pharmacokinetic parameters of $A \cup C_{t}$ and $C_{\text {max }}$, with the exception of alendronate (when drug urinary excretion was measured), where total amount of unchanged active substance in the urine excretion $\left(T_{A e}\right)$ and the maximum rate of drug excretion $\left(R_{\max }\right)$ were used(1).

The calculations were conducted using SPSS ${ }^{\circ}$ (IBM, New York, U.S.) version 20 (all data were log transformed) and EXCEL ${ }^{\circ}$ spreadsheet, and R program 3.0.1 was used to prepare graphs. To compare total or peak drug exposure, the ratios of variables in individual subjects between the first and second administration of the brand-name drug $\left(R_{2}: R_{1}\right)$, the first and second administration of the generic drug (T2:T1), and the brandname drug and the generic drug $\left(T_{1}: R_{1}\right.$ and $\left.T_{2}: R_{2}\right)$ were calculated for $A \cup C_{t}$ (or $\left.T_{A e}\right)$ and $C_{\max }$ (or $R_{\max }$ ). Since the $A \cup C_{t}$ and $C_{\max }$ of the brand-name drug $(R)$ and the generic drug (T) are both log-normally distributed, the ratios between the treatments are also lognormally distributed. The geometric coefficients of variation $\left(C V=100 \times \sqrt{e^{\sigma^{2}}-1}\right)$ of the treatment ratios $\left(R_{2}: R_{1}, T_{1}: R_{1}, T_{2}: R_{2}\right.$, and $\left.T_{2}: T_{1}\right)$ were calculated to estimate the intrasubject variability in exposure for the two drugs, and between the brand-name drug and the generic drugs at a population level.

The method of moment ( $\mathrm{MM}$ ) recommended by FDA guideline previously(12) and in the literature $(18,21)$ for investigation of individual bioequivalence was used to estimate the variance related to subject-by-formulation interaction $\left(S^{2}{ }_{D}\right)$, based on the estimated intrasubject variances of generic $\left(S^{2}{ }_{W T}\right)$ and brand-name $\operatorname{drug}\left(S_{W R}^{2}\right)$ and the variance of the difference between the average individual responses of the two drugs $\left(S_{S D}^{2}\right)$. 


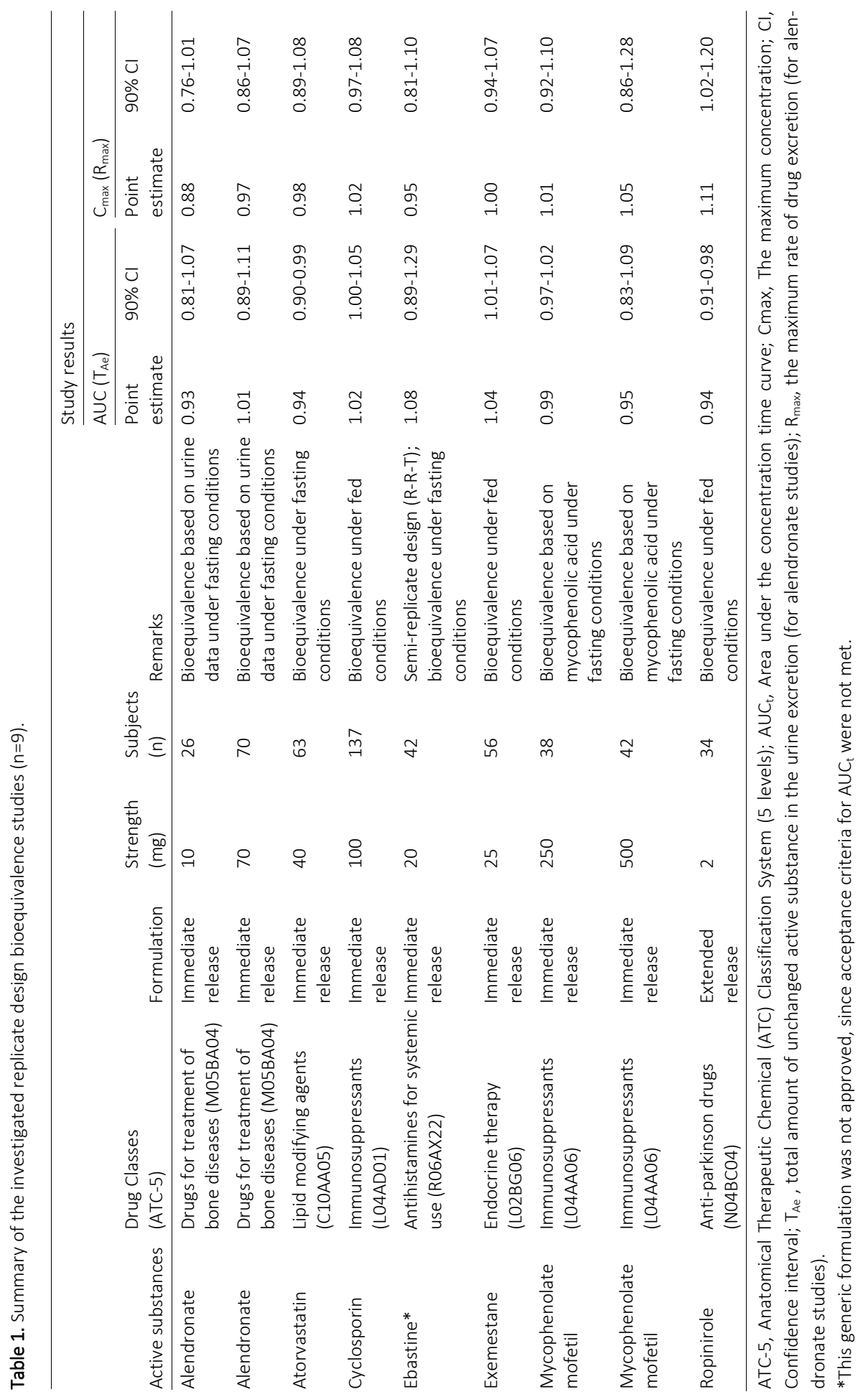


The raw data of total and peak drug exposure from the studies, and the formula described by Endrenyi, L et al. (18) for four-periods studies were used here (e.g. $\left.S_{D}^{2}=S_{S D}^{2}-\left(S_{W T}^{2}+S_{W R}^{2}\right) / 2\right)$. The upper boundary of the $95 \% \mathrm{Cl}$ for $\mathrm{S}_{\mathrm{D}}^{2}$ was also calculated, and was subsequently used to estimate as the worst case scenario the probability of having an individual exposure ratio beyond the borders of the $80-125 \%$ acceptance range upon switching from generic to brand-name drugs, compared with the probability upon repeated administration of the same drug. In addition, difference plots were used to visualize the variation in exposure in individual subjects. For this purpose, the generic:brand-name ratios $\left(T_{1}: R_{1}\right.$ and $\left.T_{2}: R_{2}\right)$ were normalized against either the brand-name ratio $\left(R_{2}: R_{1}\right)$ or the generic ratio $\left(T_{2}: T_{1}\right)$. Lastly, it was investigated whether the variations in exposure for the brand-name and the generic ratios were correlated.

\section{RESULTS}

In general, bioequivalence studies found a marked individual difference in total or peak drug exposure after repeated administration of either the brand-name drug or the generic drug (Figure 1 graph $A$ and $D$ for representative example, and Supplementary Figure I for all investigated drugs). To illustrate this, a representative series of plasma concentration-time curves obtained for repeated administration of brand-name and generic formulations are shown in Figure 2.

\section{Variability in total and peak exposure at a population level}

In the trial population, the distribution of subjects' $A \cup C_{t}$ and $C_{\max }$ values for either brand-name or generic drugs was quite comparable (for a representative distribution see Figure 1 graph A and D, and Supplementary Figure I for all investigated drugs), with there being no period effect or difference in the range of drug exposure. The coefficient of variations (CVs) of individual treatment ratios $\left(R_{2}: R_{1}, T_{1}: R_{1}, T_{2}: R_{2}\right.$ and $\left.T_{2}: T_{1}\right)$ for both $A \cup C_{t}$ and $C_{\text {max }}$ were reasonably comparable for each investigated active substance (see Table 2 with data presented in log-scale) and were not markedly different between brand-name drugs and generic drugs. The CVs of the $A \cup C_{t}$ ratio for the generic drugs $\left(T_{2}: T_{1}\right)$ and for the brand-name drugs $\left(R_{2}: R_{1}\right)$ differed by less than $10 \%$, except for alendronate $10 \mathrm{mg}$ tablets, where the CV was larger for the generic drug (77\%) than for the brand-name drug (57\%). The CVs for the $\mathrm{C}_{\max }$ ratio were also similar for the generic and brand-name drugs except for atorvastatin and ropinirole the CVs were higher for generic drug than for brand-name drug ( $97 \%$ vs. $65 \%$ and $42 \%$ vs. $21 \%$ respectively). On the other hand, for both mycophenolate studies the CVs for generic drug were lower than for brand-name drug ( $46 \%$ vs. $56 \%$ and $46 \%$ vs. $70 \%$ respectively). 

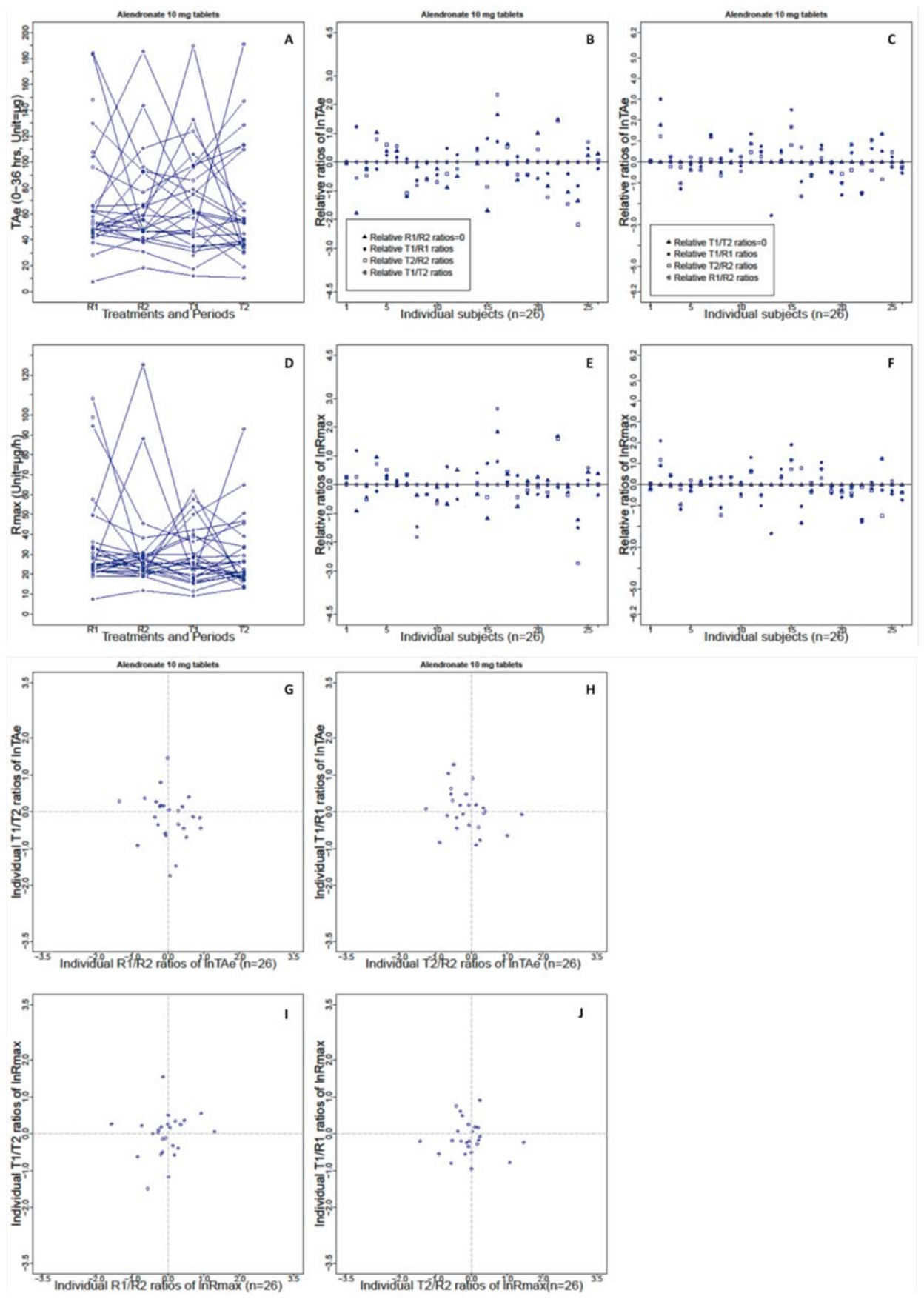

Figure 1. Intrasubject variability in the total amount of unchanged active substance excreted in the urine $\left(\mathrm{T}_{\mathrm{Ae}}\right)$ and the maximum rate of drug excretion $\left(R_{\max }\right)$ seen with brand-name and generic drugs of alendronate (10 $\mathrm{mg}$ ) in a replicate design bioequivalence study $(n=26)$. Legend. (A) Distribution of $T_{A e}$ in the trial population after administration of the brand-name or generic drug of alendronate $\left(R_{1}, R_{2}, T_{1}\right.$ or $\left.T_{2}\right)$; lines indicate the $T_{A e}$ 
levels per subject; (B) Difference plots for the In-transformed treatment ratios for $T_{A e}\left(T_{1}: R_{1}, T_{2}: R_{2}\right.$ and $\left.T_{1}: T_{2}\right)$, corrected by the brand-name drug ratios $\left(R_{1}: R_{2}\right)$ in individuals; (C) Difference plots for the ratios corrected by the generic drug ratios $\left(T_{1}: T_{2}\right) ;(G)$ Correlation of the generic ratios (Y-axis) and the brand-name ratios (X-axis) for $T_{\text {Ae }}$ on a logarithmic scale; $(H)$ Correlation of generic : brand-name ratios $\left(T_{1}: R_{1}\right.$ and $\left.T_{2}: R_{2}\right)$ after the first and second drug administration. (D, E, F, I and J) Graphs for $R_{\max }$ in the same sequence for $T_{\text {Ae. }}$. Intrasubject variability based on the other eight bioequivalence studies is shown in Supplementary Figure I-III.
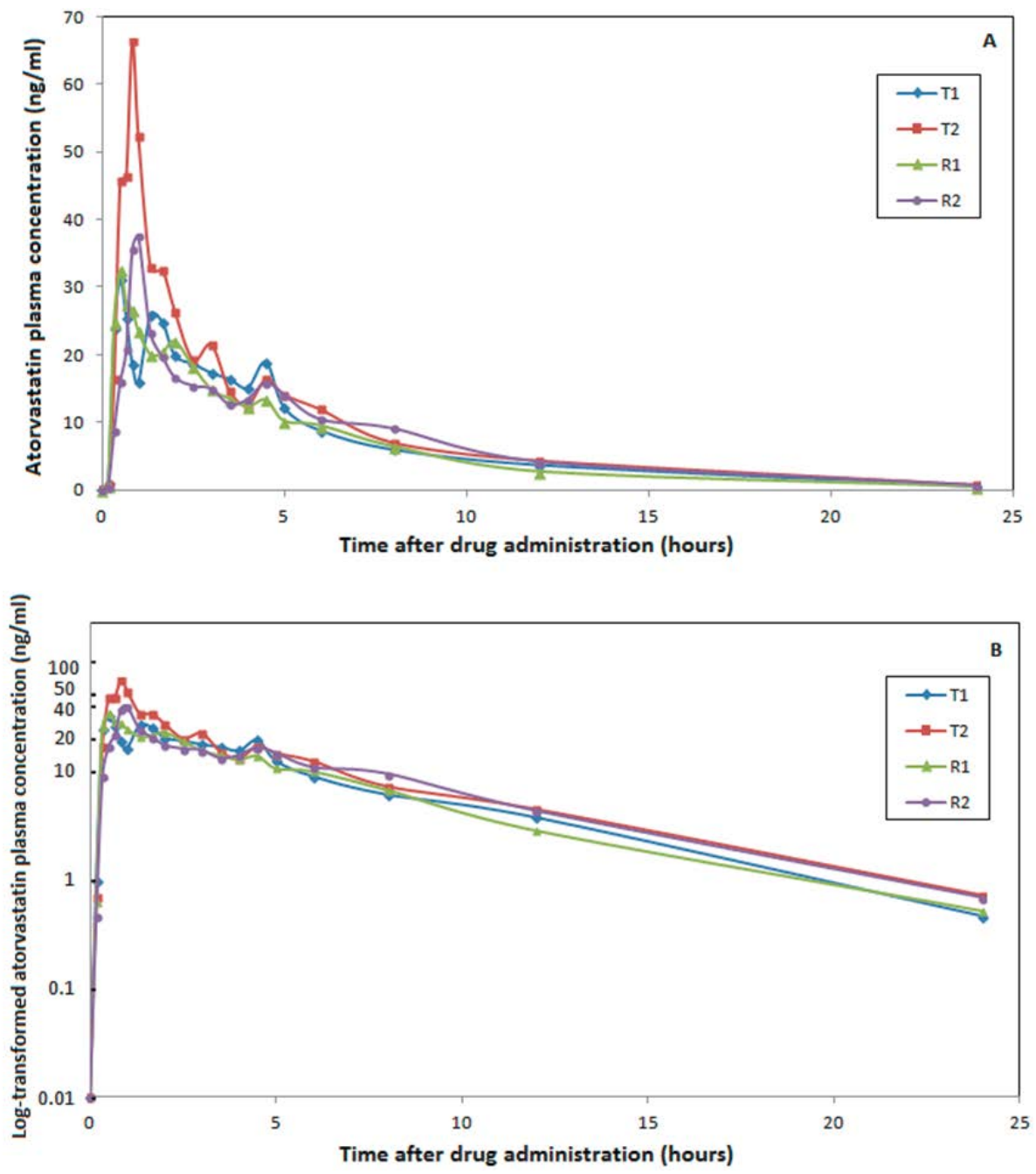

Figure 2. Individual illustrative atorvastatin plasma concentration-time curves for the brand-name and generic drugs in a single subject in the replicate design bioequivalence study ( $t=24$ hours) in an arithmetic scale (A) and in a semi-logarithmic plot (B). Legend. ' $T_{1}$ ' and ' $T_{2}$ ' represent the first and the second administration of the generic atorvastatin drug to the subject. The plasma concentration ( $Y$-axis) at every sampling time ( $\mathrm{X}$-axis) is shown; ' $R_{1}$ ' and ' $R_{2}$ ' represent the first and the second administration of the brand-name drug of atorvastatin. The mean predose level for each drug is indicated at Time point $=0$. 
Table 2. Summary of individual ratios of $\mathrm{AUC}_{0-\mathrm{t}}$ and $\mathrm{C}_{\max }$ (In-scale) at a population level.

\begin{tabular}{|c|c|c|c|c|c|c|c|c|c|c|}
\hline \multirow{2}{*}{$\begin{array}{l}\text { Active substances } \\
\text { (Strength) }\end{array}$} & \multirow[b]{2}{*}{$N$} & \multirow{2}{*}{$\begin{array}{l}\text { Ratios } \\
\text { (In-scale) }\end{array}$} & \multicolumn{4}{|l|}{$A \cup C_{0-t}$} & \multicolumn{4}{|l|}{$\mathrm{C}_{\max }$} \\
\hline & & & Mean & Min. & Max. & $\mathrm{CV}$ & Mean & Min. & Max. & $\mathrm{CV}$ \\
\hline \multirow{4}{*}{$\begin{array}{l}\text { Alendronate* } \\
\text { (10 mg) }\end{array}$} & 25 & R2/R1 & 0.02 & -1.35 & 0.91 & 0.57 & -0.07 & -1.58 & 1.29 & 0.60 \\
\hline & 26 & $\mathrm{~T} 1 / \mathrm{R} 1$ & -0.02 & -1.67 & 1.29 & 0.72 & -0.17 & -1.71 & 0.91 & 0.61 \\
\hline & 25 & $\mathrm{~T} 2 / \mathrm{R} 2$ & -0.12 & -1.26 & 1.41 & 0.63 & -0.08 & -1.43 & 1.46 & 0.60 \\
\hline & 26 & $\mathrm{~T} 2 / \mathrm{T} 1$ & -0.11 & -1.73 & 1.46 & 0.77 & -0.01 & -1.49 & 1.55 & 0.66 \\
\hline \multirow{4}{*}{$\begin{array}{l}\text { Alendronate* } \\
\text { (70 mg) }\end{array}$} & 68 & $\mathrm{R} 2 / \mathrm{R} 1$ & -0.04 & -2.06 & 1.42 & 0.78 & -0.13 & -2.65 & 1.17 & 0.89 \\
\hline & 70 & $\mathrm{~T} 1 / \mathrm{R} 1$ & -0.04 & -1.78 & 2.77 & 0.92 & -0.12 & -1.60 & 2.18 & 0.92 \\
\hline & 67 & $\mathrm{~T} 2 / \mathrm{R} 2$ & 0.03 & -1.81 & 1.41 & 0.72 & 0.04 & -1.73 & 1.57 & 0.83 \\
\hline & 67 & $\mathrm{~T} 2 / \mathrm{T} 1$ & 0.01 & -1.78 & 1.33 & 0.75 & 0.01 & -1.84 & 1.36 & 0.84 \\
\hline \multirow{4}{*}{$\begin{array}{l}\text { Atorvastatin } \\
\text { (40 mg) }\end{array}$} & 54 & $\mathrm{R} 2 / \mathrm{R} 1$ & 0.10 & -0.73 & 0.92 & 0.33 & -0.06 & -1.32 & 1.42 & 0.65 \\
\hline & 63 & $\mathrm{~T} 1 / \mathrm{R} 1$ & -0.07 & -1.05 & 0.42 & 0.28 & -0.12 & -1.56 & 2.71 & 0.71 \\
\hline & 54 & $\mathrm{~T} 2 / \mathrm{R} 2$ & -0.03 & -0.71 & 0.90 & 0.30 & 0.15 & -0.98 & 1.24 & 0.64 \\
\hline & 58 & $\mathrm{~T} 2 / \mathrm{T} 1$ & 0.13 & -0.65 & 1.48 & 0.36 & 0.22 & -3.32 & 2.52 & 0.97 \\
\hline \multirow{4}{*}{$\begin{array}{l}\text { Cyclosporin } \\
\text { (100 mg) }\end{array}$} & 133 & $\mathrm{R} 2 / \mathrm{R} 1$ & 0.01 & -0.72 & 0.86 & 0.28 & -0.02 & -1.44 & 1.57 & 0.64 \\
\hline & 137 & $\mathrm{~T} 1 / \mathrm{R} 1$ & 0.02 & -0.51 & 0.76 & 0.24 & 0.00 & -1.35 & 1.46 & 0.53 \\
\hline & 133 & $\mathrm{~T} 2 / \mathrm{R} 2$ & 0.03 & -0.55 & 0.61 & 0.24 & 0.05 & -1.50 & 1.48 & 0.58 \\
\hline & 134 & $\mathrm{~T} 2 / \mathrm{T} 1$ & 0.02 & -0.86 & 0.77 & 0.27 & 0.03 & -1.60 & 1.49 & 0.62 \\
\hline \multirow{2}{*}{$\begin{array}{l}\text { Ebastine } \\
\text { (20 mg) }\end{array}$} & 42 & $\mathrm{R} 2 / \mathrm{R} 1$ & -0.28 & -1.98 & 1.91 & 0.99 & -0.09 & -1.37 & 1.40 & 0.80 \\
\hline & 42 & $\mathrm{~T} 1 / \mathrm{R} 1$ & -0.07 & -1.80 & 1.92 & 0.88 & -0.10 & -1.40 & 1.50 & 0.70 \\
\hline \multirow{4}{*}{$\begin{array}{l}\text { Exemestane } \\
\text { ( } 25 \mathrm{mg})\end{array}$} & 54 & $\mathrm{R} 2 / \mathrm{R} 1$ & -0.02 & -0.50 & 0.44 & 0.20 & -0.02 & -0.92 & 0.94 & 0.45 \\
\hline & 56 & $\mathrm{~T} 1 / \mathrm{R} 1$ & 0.03 & -0.37 & 0.45 & 0.18 & -0.04 & -0.76 & 1.31 & 0.48 \\
\hline & 54 & $\mathrm{~T} 2 / \mathrm{R} 2$ & 0.05 & -0.32 & 0.37 & 0.15 & 0.05 & -0.99 & 0.69 & 0.38 \\
\hline & 54 & $\mathrm{~T} 2 / \mathrm{T} 1$ & 0.00 & -0.42 & 0.38 & 0.20 & 0.07 & -0.74 & 0.79 & 0.42 \\
\hline \multirow{4}{*}{$\begin{array}{l}\text { Mycophenolate } \\
\text { mofetil } \\
(250 \mathrm{mg})\end{array}$} & 37 & $\mathrm{R} 2 / \mathrm{R} 1$ & 0.00 & -0.36 & 0.24 & 0.15 & 0.05 & -1.03 & 1.68 & 0.56 \\
\hline & 38 & $\mathrm{~T} 1 / \mathrm{R} 1$ & 0.02 & -0.22 & 0.33 & 0.12 & 0.03 & -1.20 & 1.38 & 0.51 \\
\hline & 37 & $\mathrm{~T} 2 / \mathrm{R} 2$ & -0.02 & -0.43 & 0.23 & 0.14 & -0.01 & -0.68 & 1.21 & 0.42 \\
\hline & 37 & $\mathrm{~T} 2 / \mathrm{T} 1$ & -0.03 & -0.48 & 0.38 & 0.17 & 0.01 & -0.86 & 0.88 & 0.46 \\
\hline \multirow{4}{*}{$\begin{array}{l}\text { Mycophenolate } \\
\text { Mofetil } \\
\text { (500 mg) }\end{array}$} & 41 & $\mathrm{R} 2 / \mathrm{R} 1$ & 0.00 & -1.05 & 0.39 & 0.23 & 0.11 & -1.26 & 1.73 & 0.70 \\
\hline & 42 & $\mathrm{~T} 1 / \mathrm{R} 1$ & 0.01 & -0.44 & 0.43 & 0.16 & 0.11 & -0.96 & 1.54 & 0.59 \\
\hline & 40 & $\mathrm{~T} 2 / \mathrm{R} 2$ & -0.03 & -0.50 & 0.87 & 0.21 & -0.09 & -1.23 & 1.04 & 0.50 \\
\hline & 40 & $\mathrm{~T} 2 / \mathrm{T} 1$ & -0.04 & -0.37 & 0.22 & 0.13 & -0.06 & -1.12 & 0.75 & 0.46 \\
\hline \multirow{4}{*}{$\begin{array}{l}\text { Ropinirole } \\
(2 \mathrm{mg})\end{array}$} & 33 & $\mathrm{R} 2 / \mathrm{R} 1$ & -0.01 & -0.40 & 0.29 & 0.17 & 0.04 & -0.29 & 0.45 & 0.21 \\
\hline & 31 & $\mathrm{~T} 1 / \mathrm{R} 1$ & -0.06 & -0.41 & 0.31 & 0.17 & 0.09 & -0.53 & 0.70 & 0.36 \\
\hline & 31 & $\mathrm{~T} 2 / \mathrm{R} 2$ & -0.05 & -0.43 & 0.40 & 0.16 & 0.15 & -0.59 & 0.87 & 0.41 \\
\hline & 29 & $\mathrm{~T} 2 / \mathrm{T} 1$ & 0.01 & -0.33 & 0.32 & 0.18 & 0.14 & -0.70 & 0.89 & 0.42 \\
\hline
\end{tabular}

$A \cup C_{0-t}$, area under the drug concentration-time curve from time zero to the last sampling time point; $C_{\max }$, peak plasma concentration; $N$, number of subjects; $C V$, coefficient of variation.

*Alendronate is given by total among of unchanged active substance in the urine excretion $\left(T_{\mathrm{Ae}}\right)$ and the maximum rate of drug excretion $\left(R_{\max }\right)$. 


\section{Variability in total and peak exposure at an individual level}

To investigate the difference in drug exposure in individuals, the parameters of variances used for investigation of individual bioequivalence were estimated (listed in Inscale in Table 3). Of note, the variances cannot be estimated for ebastine study due to the three-treatment study design (R-R-T). The results showed that the intrasubject variance of generic drugs (in In-scale) was comparable with that of brand-name drugs, and also comparable with the variance of switching from one to another. Further, variances related to subject-by-formulation interaction for both $A \cup C_{t}$ and $C_{\max }$ appeared relatively small $(<0.05)$ and most were negatively estimated by $\mathrm{MM}$, meaning that they were close to zero. The small subject-by-formulation interaction related variances indicate that the difference in drug exposure upon switching from brand-name to generic drug is very similar to repeated administration of the same drug for every subject. The calculated upper boundary of $95 \% \mathrm{Cl}$ for the variances of subject-by-formulation interaction was also quite small in all studies for both $A \cup C_{t}$ and $C_{\max }(<0.06$, except for alendronate $70 \mathrm{mg}$, <0.16).

In the worst scenario, in most studies, the probability of lack of bioequivalence was similar ( $<10 \%$ difference) for an individual subject upon repeated administration of the brand-name drug or switched from brand-name to generic drug. As the variances of the subject-by-formulation interaction were very small, the probability of an exposure ratio beyond the borders of the $80-125 \%$ range was predominately dependent on intrasubject variability of drugs. The difference between the formulation of generic and brandname drugs seems not contribute to the observed difference in drug exposure upon switching in individual subjects. However, for the ropinirole study, the variance of generic drug for $C_{\max }(0.08)$ was much larger than that of the brand-name drug (0.022), which leads to the probability of ratio beyond the boundary of the $80-125 \%$ range upon repeated administration of generic drug (57\%) being higher than that upon repeated administration of the brand-name drug (29\%). In addition, the probability of the ratio beyond the boundary of the $80-125 \%$ range upon switching from the generic to brandname drug (57\%) was higher than that of repeated administration of the brand-name drug. By contrast, in the mycophenolate mofetil $(500 \mathrm{mg}$ ) study, the probability of the ratio beyond the boundary of the $80-125 \%$ range upon repeated administration of the generic drug (9\%) for $A \cup C_{t}$ was much lower than that for repeated administration of the brand-name drug (32\%) because of the difference in variances (0.009 vs. 0.026). 


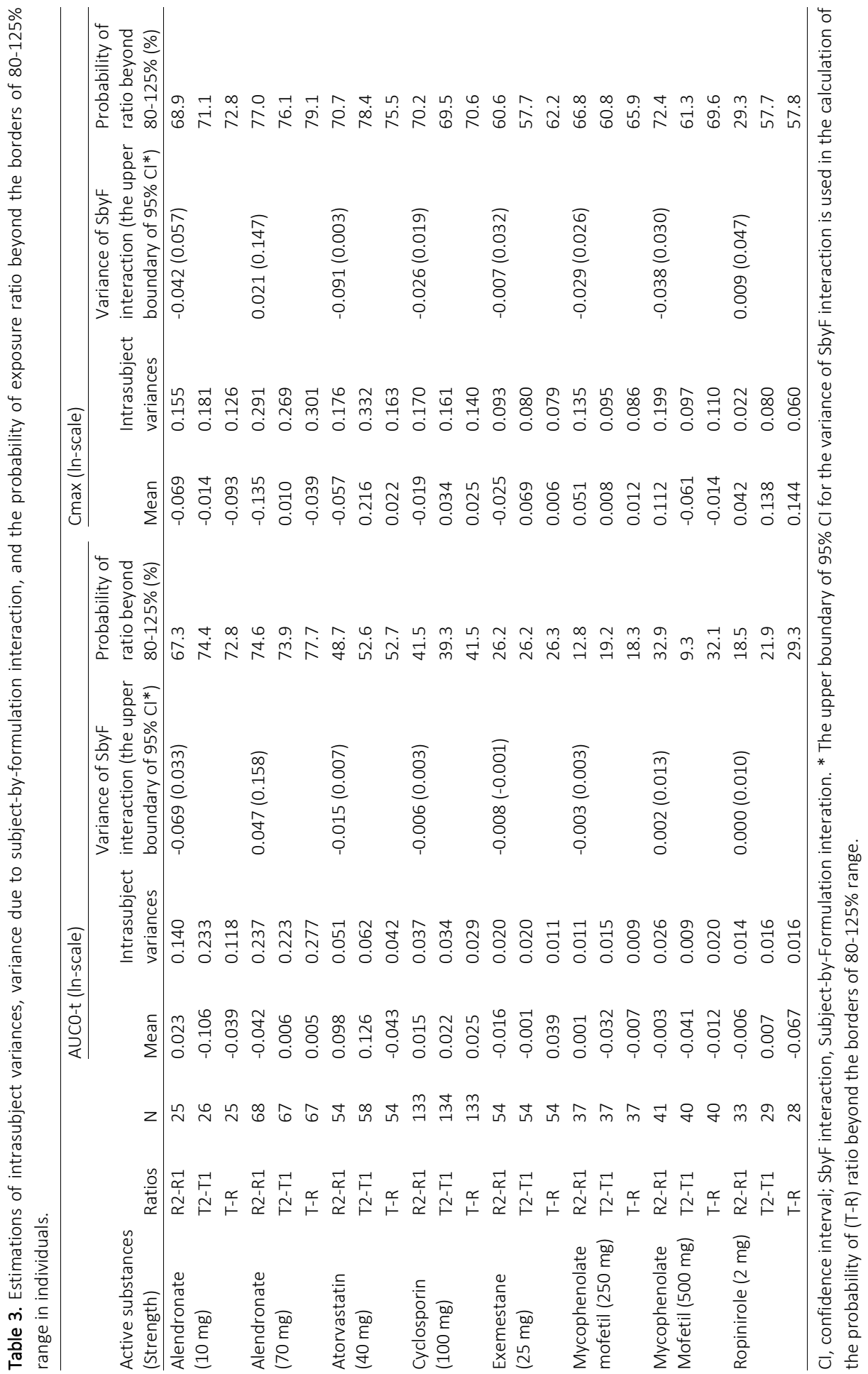


Furthermore, the variations in drug exposure $\left(A \cup C_{t}\right.$ and $C_{\max }$ ) between the four treatments $\left(T_{1}, T_{2}, R_{1}\right.$ and $\left.R_{2}\right)$ were monitored. For this purpose, the generic : brandname ratios $\left(T_{1}: R_{1}\right.$ and $\left.T_{2}: R_{2}\right)$ were corrected by subtracting the reference ratios $\left(R_{2}: R_{1}\right)$ for both $A \cup C_{t}$ and $C_{\text {max }}$ for individual subjects in each study. The graphs for the replicate study of alendronate $10 \mathrm{mg}$ tablets are shown as an example in Figure 1 (graph B and E), and the results for all studies are shown in Supplementary Figure II (graph A-F). The individually corrected generic : brand-name ratios had a comparable distribution for both $A \cup C_{t}$ and $C_{\max }$. Moreover, the corrected individual generic : brand-name ratios and the generic ratios $\left(T_{2}: T_{1}\right)$ in the study population were symmetrically distributed around the zero line for both $A \cup C_{t}$ and $C_{\max }$. Virtually identical results were obtained after correction for the observed individual ratios with $T_{2}: T_{1}$ instead of $R_{2}: R_{1}$ (Figure 1 . graph $C$ and $\mathrm{F}$ for representative example, and Supplementary Figure II. graph G-L for all investigated studies). On the basis of these data, drug exposure appeared to vary at random in individuals in a similar manner for the generic and the brand-name drugs. This hypothesis is supported by our finding that the variances for the subject-by-formulation interaction were negligible.

\section{Correlation between treatment ratios}

A comparison of individual $A \cup C_{t}$ and $C_{\max }$ ratios for generic drugs $\left(T_{2}: T_{1}\right)$ and brandname drugs $\left(R_{2}: R_{1}\right)$ is shown in Figure 1 graph $G$ and I for alendronate $10 \mathrm{mg}$ (and in Supplementary Figure III, graph A-F for all investigated drugs). In all graphs, the points were randomly scattered around the zero point, forming a circular area, which shows that the ratios for generic drugs and brand-name drugs are not correlated. It means that in the trial population the distribution of the individual variations in exposure to a generic drug was not different from a brand-name drug. Furthermore, the possible variation in exposure in the individual subjects after repeated administration of a brandname drug was comparable to that of repeated administration of the generic drug, regardless of whether data for the first or second drug administration were compared (i.e., $T_{1}: R_{1}$ vs. $T_{2}: R_{2}$ ) in Figure $1 \mathrm{H}$ and J (and in Supplementary Figure III G-L for all investigated studies), and the circular area formed by the individual generic : brand-name ratios was no different from the area in the graphs for the generic and brand-name ratios.

\section{DISCUSSION}

In this study, we attempted to explain why an individual's drug total or peak exposure is sometimes different when a brand-name drug is exchanged for a generic drug. Using data from several replicate design bioequivalence studies, we found that the variances for $A \cup C_{t}$ and $C_{\text {max }}$ of individual treatment ratios ( $T$ vs. $T, T$ vs. $R$ and $R$ vs. $R$ ) were similar 
for the seven investigated active substances. This outcome at a population level indicates that the intrasubject variability in $A \cup C_{t}$ and $C_{\max }$ is comparable upon repeated administration of brand-name drugs or repeated administration of generic drugs, and upon the exchange of brand-name and generic drugs. Therefore, in general, differences in variability of brand-name and generic drugs offer no valid explanation for the difference in individual drug exposure sometime seen after switching from a brand-name drug to a generic drug. Based on the results of the estimation of intrasubject variances using the individual bioequivalence approach, an exception to this conclusion may be ropinirole. In the ropinirole bioequivalence study included in our investigation, the intrasubject variance in $\mathrm{C}_{\max }$ of generic drug was double that of brand-name drug. However, the opposite situation has been seen in mycophenolate studies where the intrasubject variance of the generic drug was much lower than that of brand-name drug. As the variances due to the subject-by-formulation interaction can be considered negligible, the variation in an individual's exposure upon switching appears predominantly dependent on the intrasubject variances of the drug at stake. Overall, the estimations at an individual level regarding the role of intrasubject variances and subject-byformulation interaction related variance in difference in drug exposure between generic and brand-name drug were in line with our findings at a population level. This is further confirmed by the findings in the difference plots, demonstrating that the individual difference in exposure $\left(A \cup C_{t}\right.$ and $C_{\max }$ ) between the brand-name and generic drugs shows a random distribution within a range in a given population. In addition, based on the correlation graphs (Figure 1 graph $\mathrm{G}, \mathrm{H}, \mathrm{I}$ and J), the variation in exposure was similar when either one (generic or brand-name) drug was given repeatedly or when one drug was switched for another.

As a retrospective analysis, the investigation is limited by the data available. We had access to data held by the Dutch Medicines Regulatory Authority for drugs investigated in replicate design studies as part of registration requirements. For the purpose of registration of generic drugs, it is most likely that the applicants do not submit failed studies. Consequently, it is conceivable that only successful bioequivalence studies are available to regulatory authorities. Still, in our investigation, the retrieved study for ebastine failed to demonstrate bioequivalence between the generic and the brand-name drug. In that study, the ratio of $R_{2} / R_{1}$ was larger than $T_{1} / R_{1}$ for $A \cup C_{t}$, meaning that the total exposure appeared more different upon repeat the administration of the brand-name drug than following a switch to the generic drug. However, repeat administration of the generic drug $\left(T_{2} / T_{1}\right)$ was not investigated in this study. Nevertheless, for other investigated generic drugs, unidentified failed studies may exist. The impact of such potentially failed studies on our results cannot be estimated and a potential selection bias cannot be excluded." Most active substances investigated in replicate design studies are known to give rise to a highly variable peak exposure (i.e., intrasubject variability $>30 \%$ for $\left.C_{\max }\right)(1)$, and this is why these studies are necessary for regulatory purposes. The active substances investigated covered various therapeutic areas. Although e.g., the generic 
drugs for epilepsy and other reported medicines in literature $(3,4)$ were not investigated, there seems no a-priori reason to assume that a different result would be obtained for these drugs from different therapeutic area. Similarly, though for this study only replicate design studies for drugs with a high intrasubject variability were available, we do not expect different results for drugs with a low intrasubject variability. Also for these low variable drugs intrasubject variability is likely to explain the major part of the differences in exposure that are obtained following switching between brand-name and generic drugs. A third limitation is that all studies involved healthy volunteers, and so did not mimic the actual clinical situation in a patient setting. It is known that drug exposure and pharmacokinetic variability can differ in patients and healthy subjects, for multiple physiological reasons. However, the principal cause of variation in exposure after repeated administration or switching from one (brand-name or generic) drug to another as identified in this study, namely, intrasubject variability in exposure, though potentially increased in patient, is expected to be comparable in patients and in healthy subjects. Thus in this sense, findings for healthy volunteers can be extrapolated to patient populations. In addition, although single-dose study conditions do not mimic the actual clinical situation (i.e. often involving multiple-dose towards a steady state), a single dose study is considered to be more sensitive for detecting differences in drug exposure between generic and brand-name drugs than a multiple-dose/steady-state study(1). Thus, if bioequivalence is demonstrated under single-dose conditions, the generic drug is also assumed bioequivalent with the brand-name drug under steady state conditions as well. Therefore, the single-dose replicate design studies used in our investigation are also considered relevant and sensitive for investigating the issue regarding intrasubject variability in clinical practise.

In the literature it is suggested that, although bioequivalence has been demonstrated at a population level, at an individual patient level exposure following a switch to a generic drug may be different, presumably due to differences between the brand-name drug and the generic drug (22). While we found differences in exposure (either increased or decreased) after switching drugs, we do not agree that this difference is primarily due to differences between the formulation of the brand-name and generic drugs. Instead, our results clearly indicate that the difference in exposure after switching to a generic drug is almost exclusively due to the intrasubject variability in the pharmacokinetics of the active substance, independent of the formulation used - the variation in exposure was similar whether repeated administrations of the same (brandname or generic) drug were given or one (brand-name or generic)drug was switched for another in a population level and also identified in an individual level. This variation in exposure after repeated administration of the same (either brand-name or generic) drug has not been acknowledged in the literature. In our current investigation using the individual bioequivalence approach, the variance related to subject-by-formulation interaction can be considered negligible. Even in the worst case scenario, the subjectby-formulation interaction did not affect the probability of obtaining an equivalent 
exposure when switching from brand-name to generic drug, and thus this probability was comparable with that obtained upon repeated administration of the same drug. This supports one of the reasons to withdrawn the FDA guideline that a potential lack of interchangeability due to subject-by-formulation interaction in average bioequivalence was not considered to be sufficiently substantiated. Furthermore, our findings are in line with those of a clinical cohort study involving patients with epilepsy, in which there was an increased risk of seizures when prescriptions were refilled, regardless of whether the same brand-name drug was prescribed or a generic drug was substituted (23). In addition, our findings do not support substantial intersubject differences in the $A \cup C_{t}$ and $C_{\max }$ ratios when comparing brand-name and generic drugs (among $T$ vs. $T, T$ vs. $R$, and $R$ vs. R), as postulated by Bialer et al(13), but instead propose that the difference in exposure is based on a random intrasubject variation in exposure upon repeated administration of one drug (either brand-name or generic).

In conclusion, in the investigated 9 studies, the variation in total and peak drug exposure seen in individual patients after switching to a generic drug is generally comparable with the variation in exposure seen with repeated administration of a brand-name drug. In such cases, only the intrasubject variability in the pharmacokinetics of the active substance seems play a crucial and decisive role in the variation in drug exposure seen in individuals when switching from a brand-name drug to a generic drug. No additional effect of the formulation of generic drugs on the variation in drug exposure was identified. Differences in exposure observed after switching from a brand-name to a generic drug are therefore generally within the range of exposures observed upon repeated administration of the brand-name drug. Thus, from a clinical pharmacological point of view, our data support that, the benefit-risk of a generic drug is comparable to that of the brand-name drug for the medicines that were included in this investigation. Further confirmatory investigation in a larger spectrum of drugs in order to broaden this conclusion to other drugs is warranted.

\section{ACKNOWLEDGMENTS}

We thank Christine Gispen-de Wied, MD, PhD, at the Medicine Evaluation Board for research support. No compensation was provided for her role in the study. 


\section{REFERENCES}

(1) Guidline on the Investigation of Bioequivalence. (Committee for Medicinal Products for Human use (CHMP), European Medicine Agency,, London, 2010).

(2) Guidance for Industry. Bioavailability and Bioequivalence. Studies for Orally Adminsitered Drug ProductsGeneral Considerations. US Department of Health and Human Services, Food and Drug Administration Center for Drug Evaluation and Research (CDER) (2003).

(3) Robertsen, I. et al. Use of Generic Tacrolimus in Elderly Renal Transplant Recipients: Precaution Is Needed. Transplantation, (2014).

(4) Borgheini, G. The bioequivalence and therapeutic efficacy of generic versus brand-name psychoactive drugs. Clin Ther 25, 1578-92 (2003).

(5) Crawford, P., Hall, W.W., Chappell, B., Collings, J. \& Stewart, A. Generic prescribing for epilepsy. Is it safe? Seizure 5, 1-5 (1996).

(6) Meredith, P.A. Potential concerns about generic substitution: bioequivalence versus therapeutic equivalence of different amlodipine salt forms. Curr Med Res Opin 25, 2179-89 (2009).

(7) Andermann, F., Duh, M.S., Gosselin, A. \& Paradis, P.E. Compulsory generic switching of antiepileptic drugs: high switchback rates to branded compounds compared with other drug classes. Epilepsia 48 , 464-9 (2007).

(8) Zachry, W.M., 3rd, Doan, Q.D., Clewell, J.D. \& Smith, B.J. Case-control analysis of ambulance, emergency room, or inpatient hospital events for epilepsy and antiepileptic drug formulation changes. Epilepsia $\mathbf{5 0 ,}$ 493-500 (2009).

(9) Bautista, R.E., Gonzales, W. \& Jain, D. Factors associated with poor seizure control and increased side effects after switching to generic antiepileptic drugs. Epilepsy Res 95, 158-67 (2011).

(10) Kesselheim, A.S. et al. Seizure outcomes following the use of generic versus brand-name antiepileptic drugs: a systematic review and meta-analysis. Drugs 70, 605-21 (2010).

(11) Kesselheim, A.S. et al. Clinical equivalence of generic and brand-name drugs used in cardiovascular disease: a systematic review and meta-analysis. JAMA : the journal of the American Medical Association 300, 2514-26 (2008).

(12) Guidance for Industry: Statistical approaches to establishing bioequivalence. (ed. U.S. Department of Health and Human Services, F.a.D.A., Center for Drug Evaluation and Research (CDER)) (2001).

(13) Bialer, M. Generic products of antiepileptic drugs (AEDs): is it an issue? Epilepsia 48, 1825-32 (2007).

(14) Guidance for industry: Average, population, and individual approaches to establishing bioequivalence (ed. U.S. Department of Health and Human Services, F.a.D.A., Center for Drug Evaluation and Research (CDER)) (1999).

(15) In Vivo bioequivalence studies based on population and individual bioequivalence approaches. . (ed. U.S. Department of Health and Human Services, F.a.D.A., Center for Drug Evaluation and Research (CDER)) (1997).

(16) Chow, S.-C. Individual bioequivalence-A review of FDA draft guidance. Drug Information Journal 33, 10 (1999).

(17) Hsuan, F.C. Some statistical considerations on the FDA draft guidance for individual bioequivalence. Statistics in medicine 19, 2879-84 (2000).

(18) Endrenyi, L., Taback, N. \& Tothfalusi, L. Properties of the estimated variance component for subject-byformulation interaction in studies of individual bioequivalence. Statistics in medicine 19, 2867-78 (2000).

(19) Bialer, M. \& Midha, K.K. Generic products of antiepileptic drugs: a perspective on bioequivalence and interchangeability. Epilepsia 51, 941-50 (2010).

(20) Hauck, W.W., Hyslop, T., Chen, M.L., Patnaik, R. \& Williams, R.L. Subject-by-formulation interaction in bioequivalence: conceptual and statistical issues. FDA Population/Individual Bioequivalence Working Group. Food and Drug Administration. Pharmaceutical research 17, 375-80 (2000).

(21) Endrenyi, L. A Simple Approach for the Evaluation of Individual Bioequivalence. Drug Information Journal 29, 847-55 (1995). 


\section{Chapter 3}

(22) van Gelder, T. Within-patient variability in immunosuppressive drug exposure as a predictor for poor outcome after transplantation. Kidney international 85, 1267-8 (2014).

(23) Gagne, J.J., Avorn, J., Shrank, W.H. \& Schneeweiss, S. Refilling and switching of antiepileptic drugs and seizure-related events. Clin Pharmacol Ther 88, 347-53 (2010). 


\section{SUPPLEMENTARY}

Figure I. Distribution of $A \cup C_{t}\left(T_{A e}\right)$ and $C_{\max }\left(R_{\max }\right)$ in the trial population after administration of the brand-name or generic drugs $\left(R_{1}, R_{2}, T_{1}\right.$ or $\left.T_{2}\right)$ in nine bioequivalence studies.
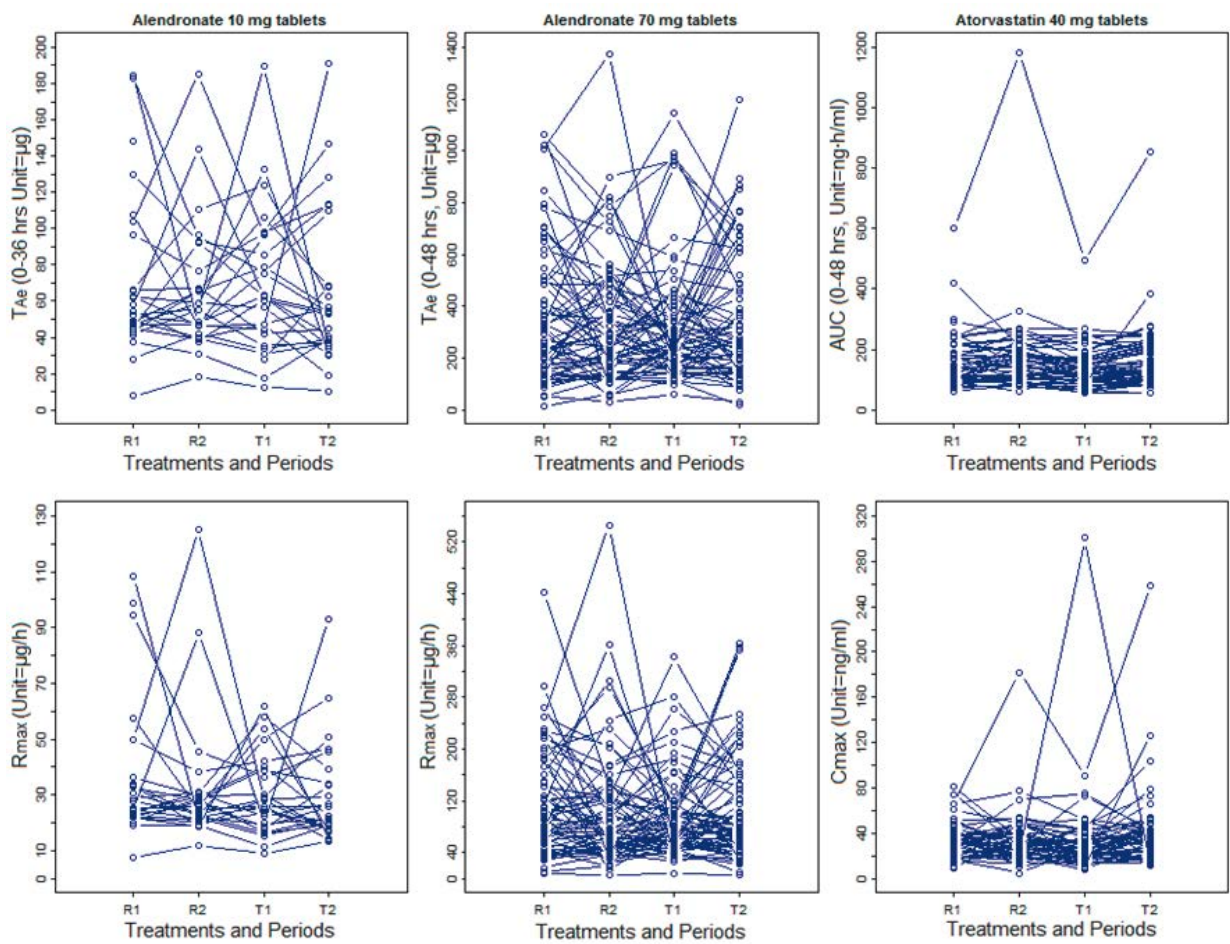

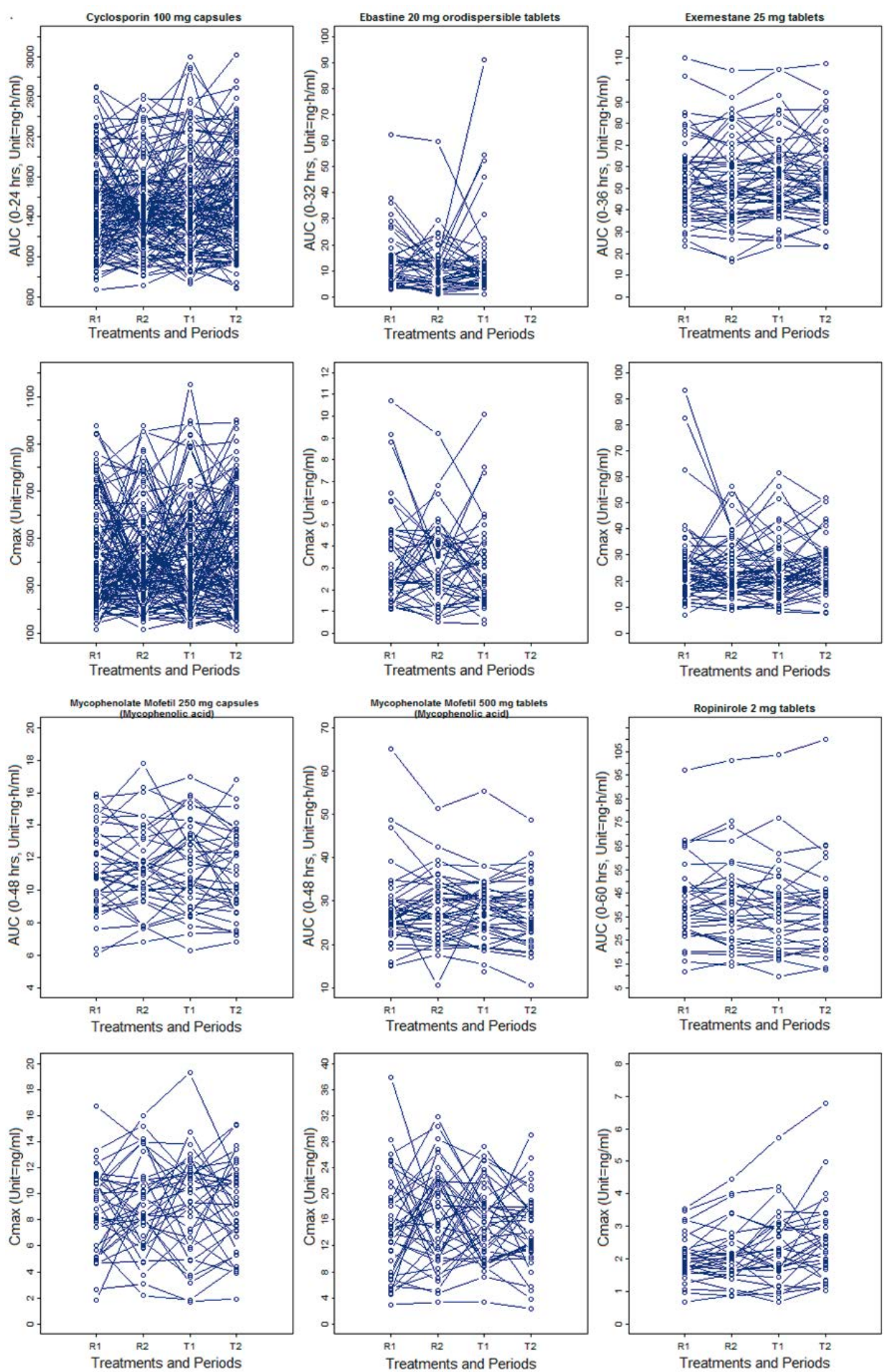
Figure II (Part 1). Difference plots for the In-transformed treatment ratios for $A \cup C_{t}\left(T_{A e}\right)$ and $C_{\max }\left(R_{\max }\right)\left(T_{1}: R_{1}\right.$, $T_{2}: R_{2}$ and $\left.T_{1}: T_{2}\right)$ corrected by the brand-name drug ratios $\left(R_{1}: R_{2}\right)$ in individuals.
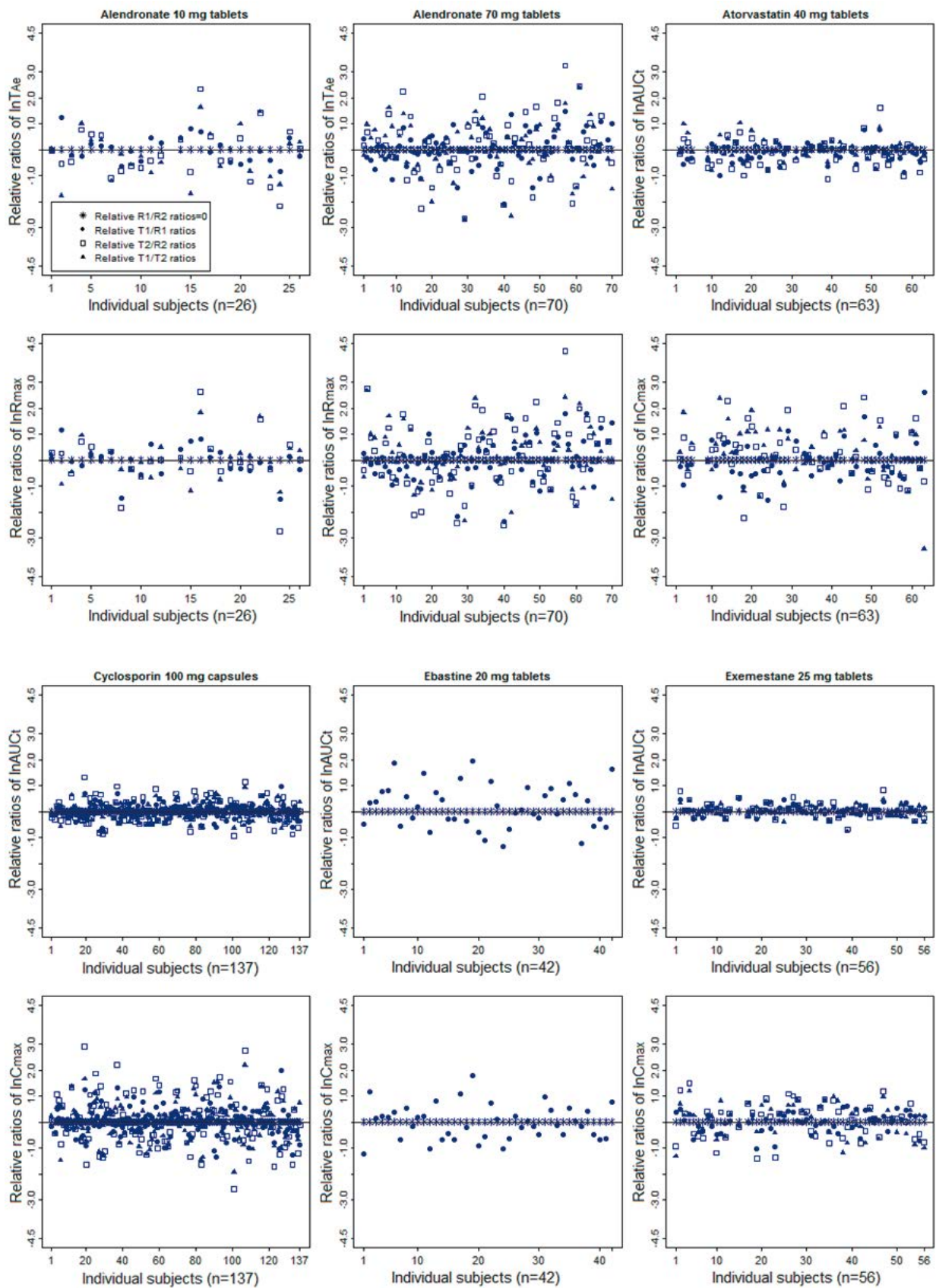

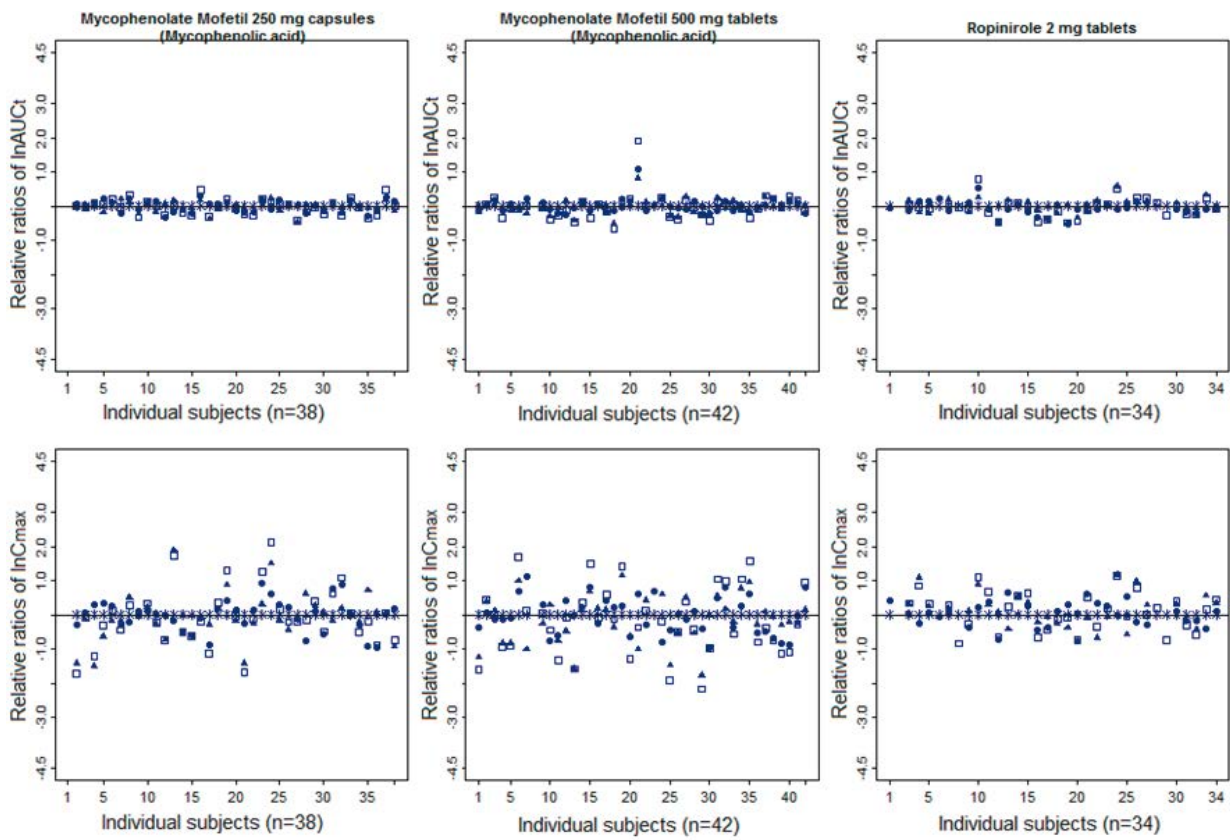

Figure II (Part 2). Difference plots for the In-transformed treatment ratios for $A \cup C_{t}\left(T_{A e}\right)$ and $C_{\text {max }}\left(R_{\max }\right)\left(T_{1}: R_{1}\right.$, $T_{2}: R_{2}$ and $\left.T_{1}: T_{2}\right)$ corrected by the generic drug ratios $\left(T_{1}: T_{2}\right)$ in individuals.
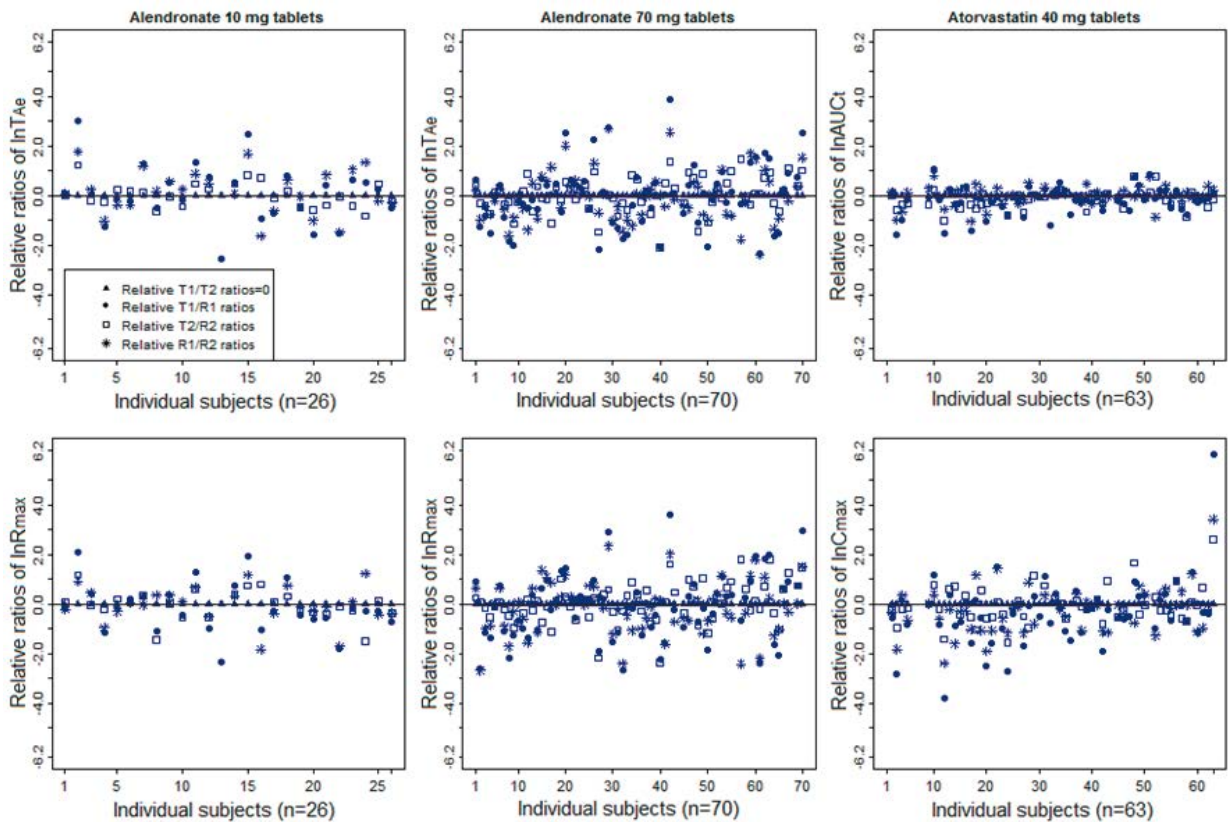

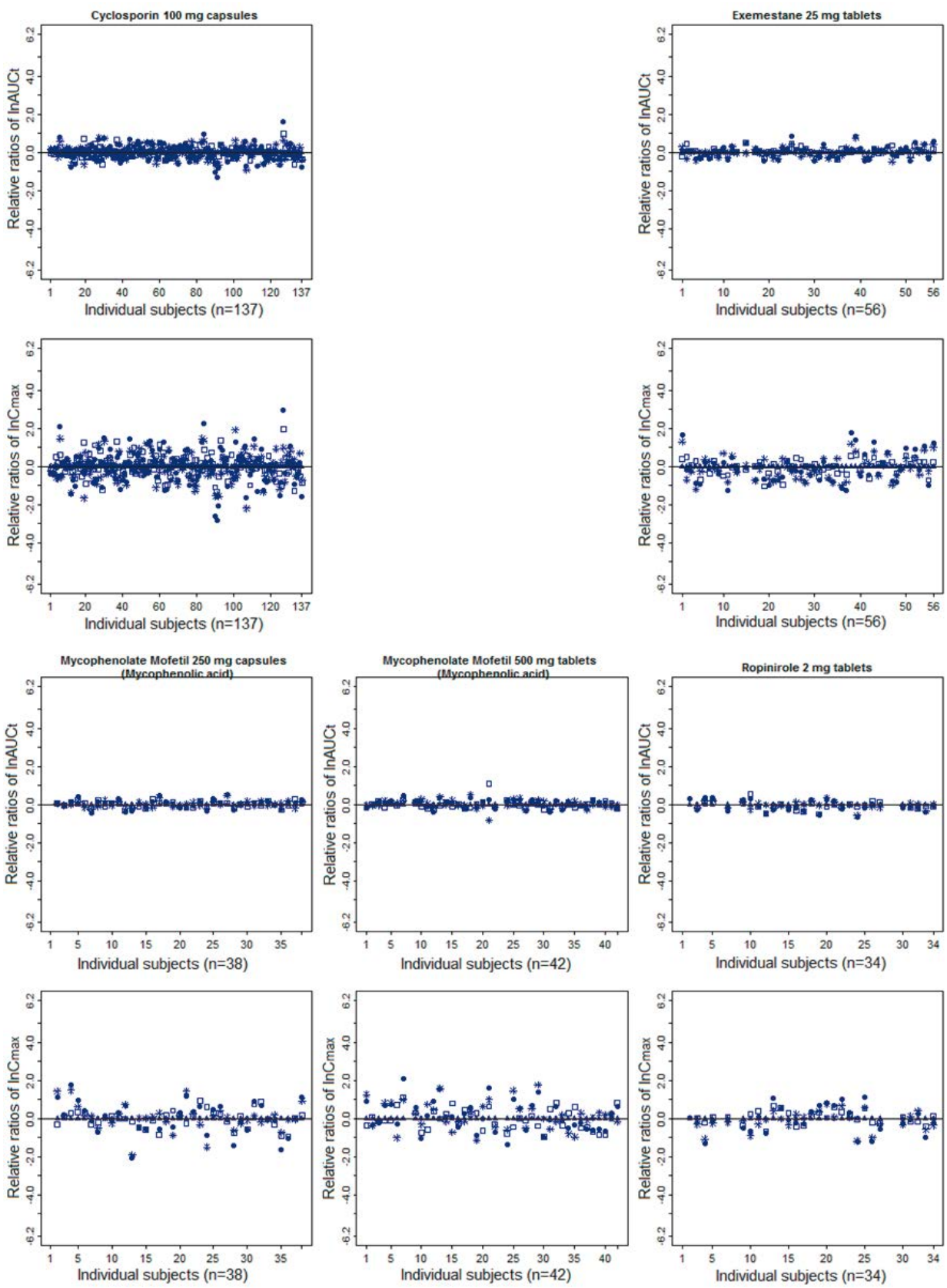


\section{Chapter 3}

Figure III (Part 1). Correlation of the generic ratios and the brand-name ratios for $A \cup C_{t}\left(T_{A e}\right)$ and $C_{\max }\left(R_{\max }\right)$ on a logarithmic scale.
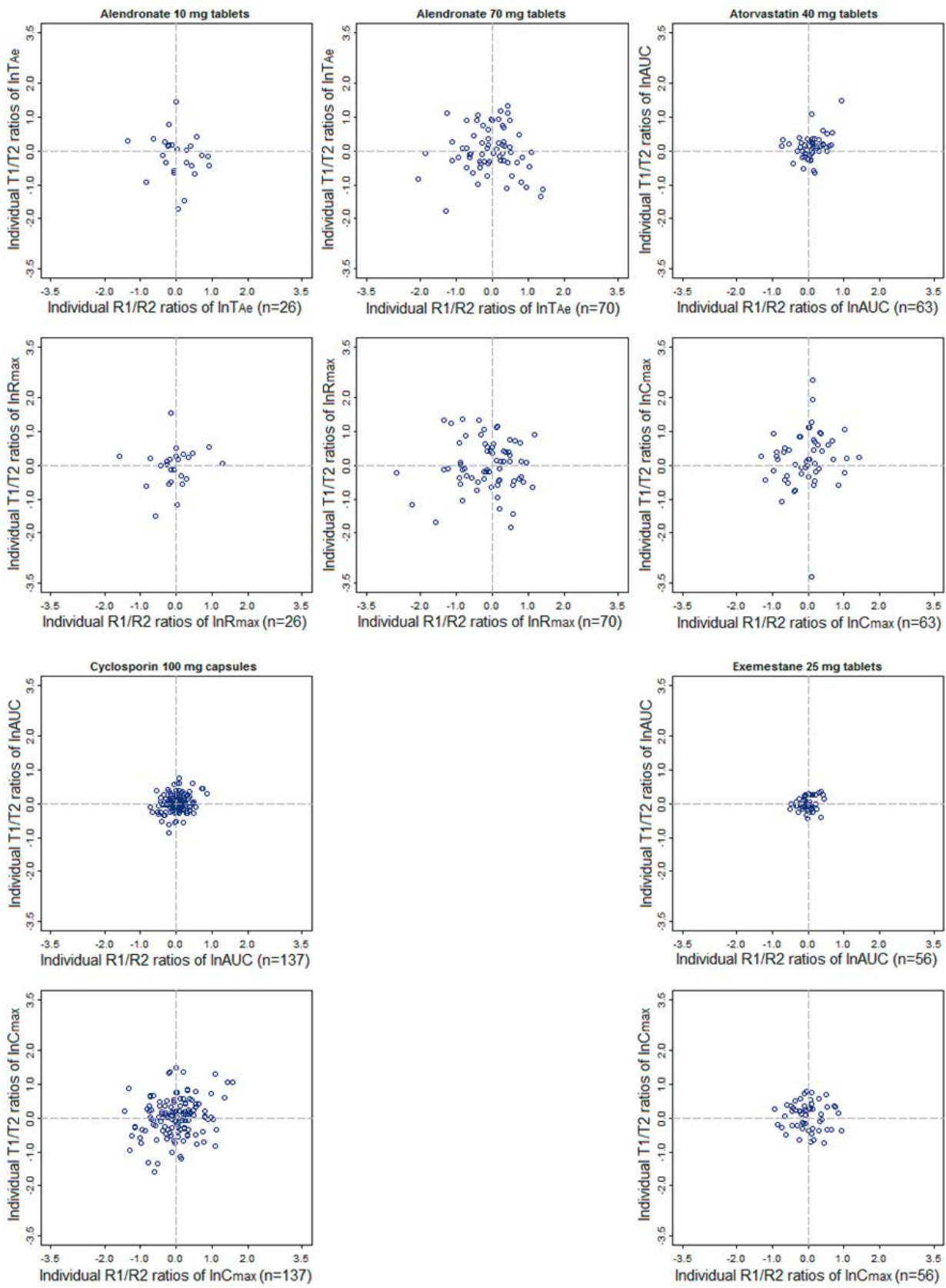

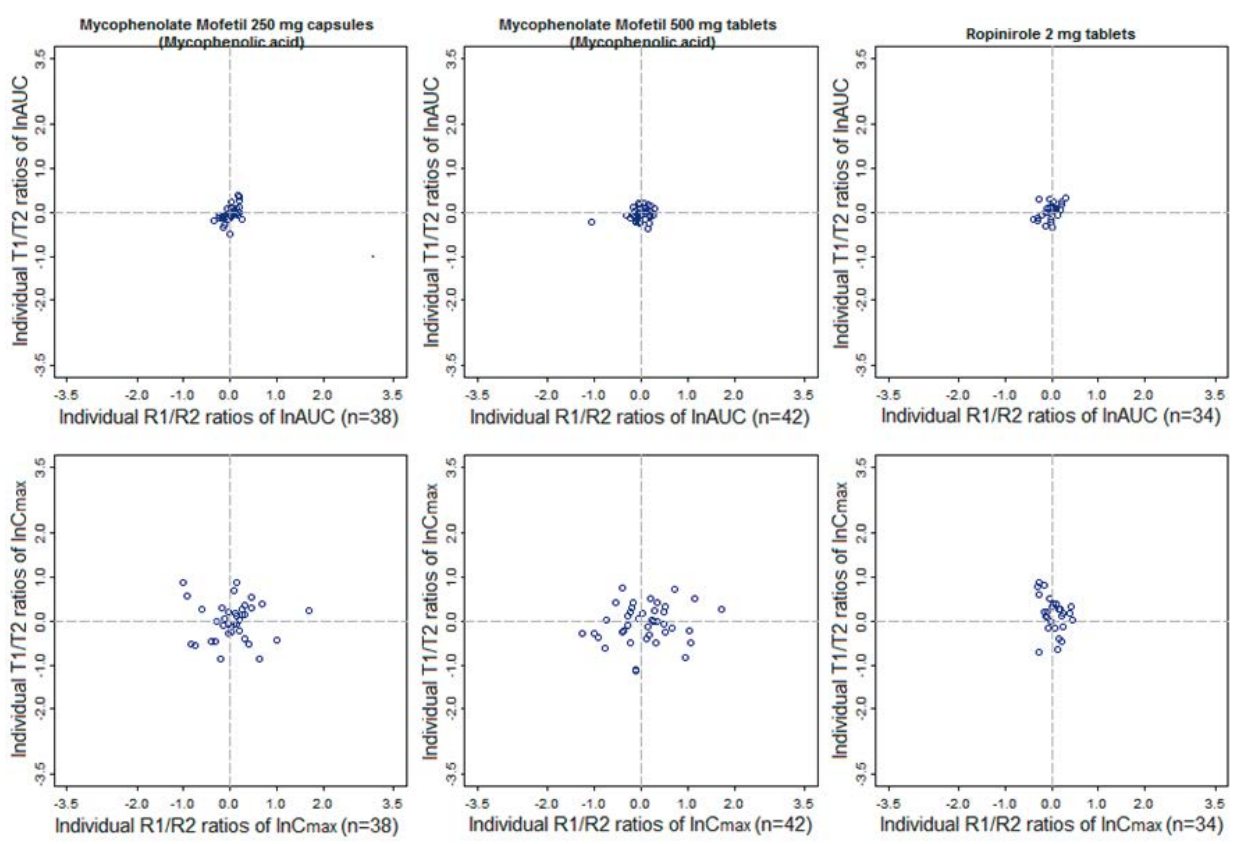

Figure III (Part 2). Correlation of generic:brand-name ratios $\left(T_{1}: R_{1}\right.$ and $\left.T_{2}: R_{2}\right)$ after the first and second drug administration for $A \cup C_{t}\left(T_{A e}\right)$ and $C_{\max }\left(R_{\max }\right)$ on a logarithmic scale.
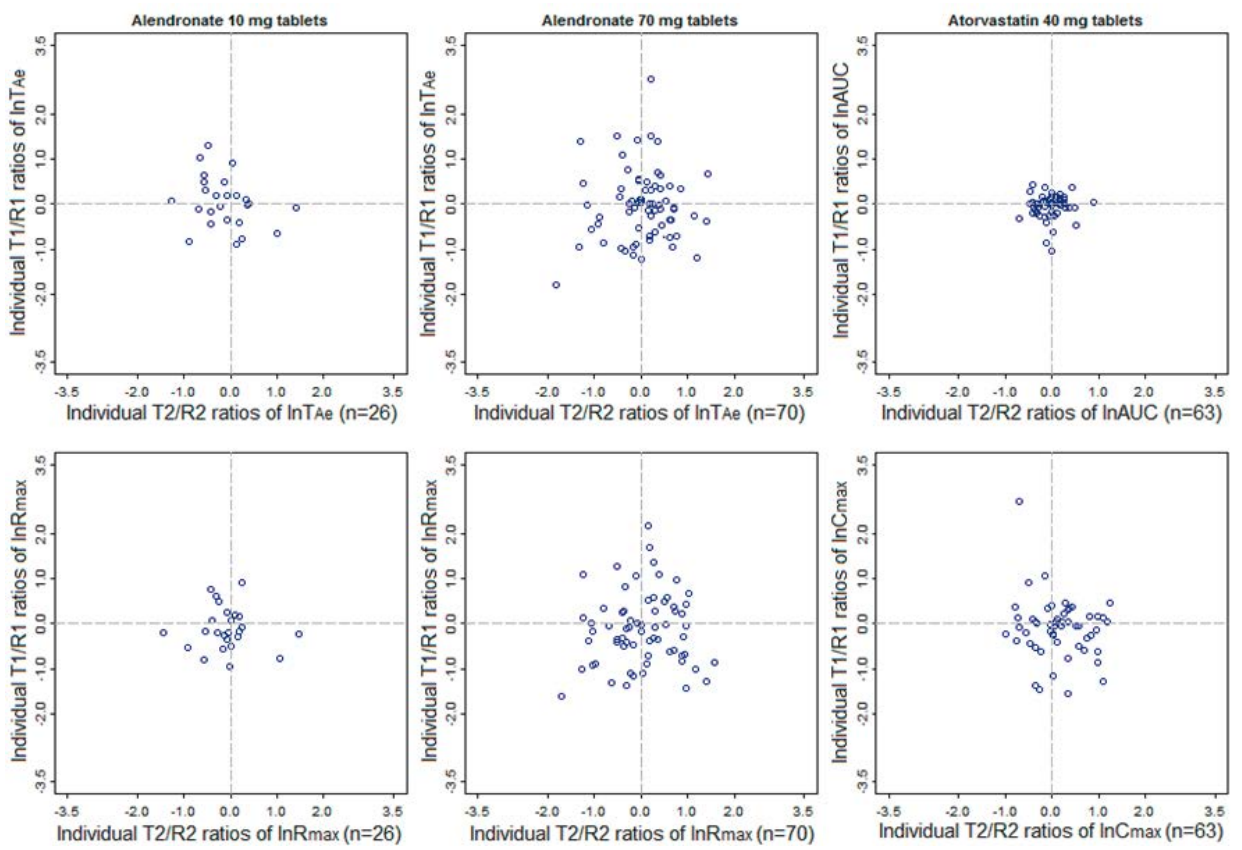

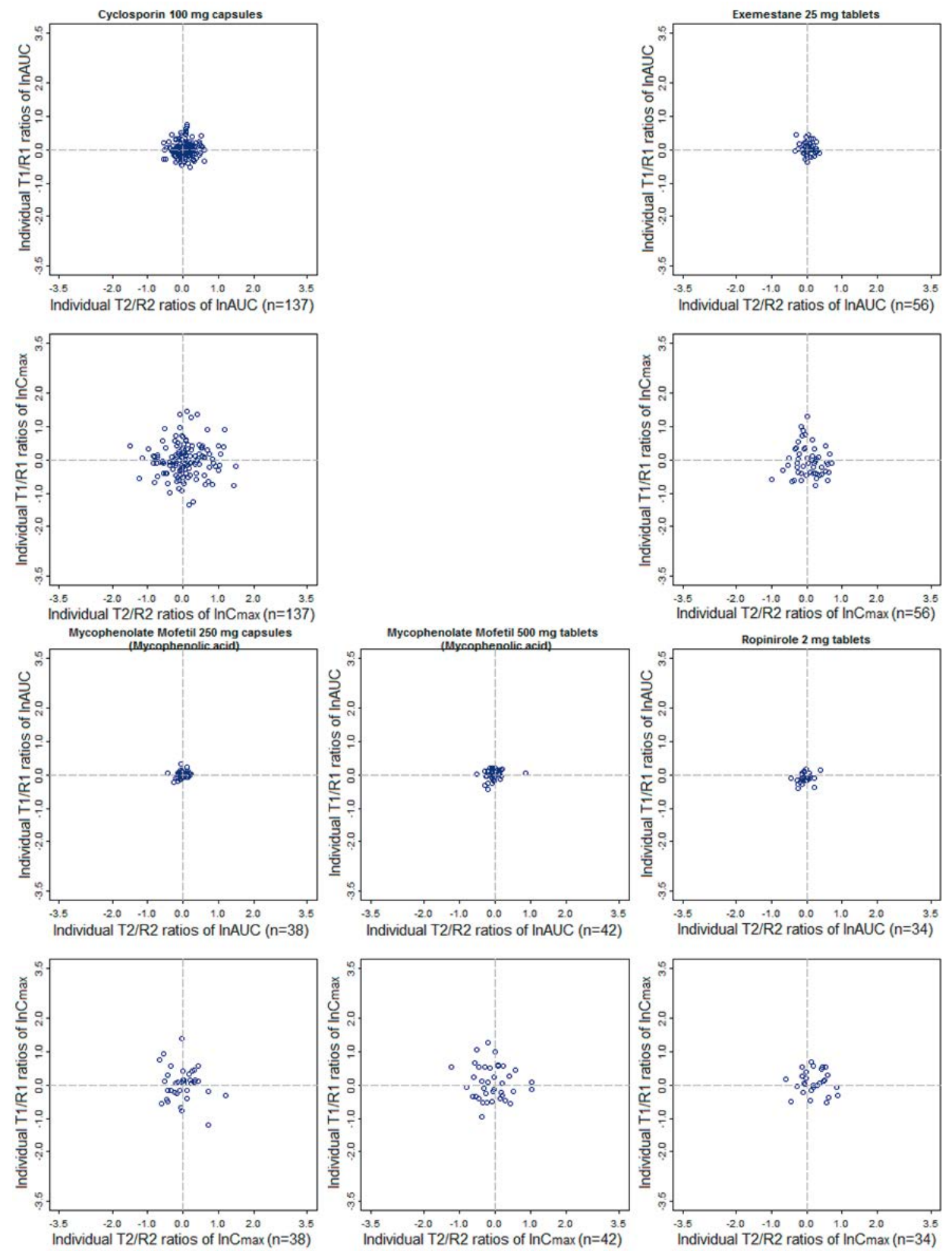


\section{Chapter}

\section{The cumulative impact of post-marketing variations on interchangeability of generic drugs: a model for assessing bioequivalence}

$\underline{\text { Yang } \mathrm{Yu}^{1,2}}$, Amr Makady $^{3}$, Peter Jongen ${ }^{2}$, Steven Teerenstra ${ }^{2,4}$, Cees Neef ${ }^{2,5}$, David Burger $^{6}$ and Marc Maliepaard ${ }^{2}$

${ }^{1}$ Dept. Toxicology, CARIM, Maastricht University Medical Centre, Maastricht, The Netherlands

${ }^{2}$ Medicines Evaluation Board, Utrecht, The Netherlands

${ }^{3}$ UIPS, Utrecht University, The Netherlands

${ }^{4}$ Dept. for Health Evidence, section Biostatistics, Radboud University Medical Center, Nijmegen, The Netherlands

${ }^{5}$ Dept. Toxicology, CAPHRI, Maastricht University Medical Centre, Maastricht, The Netherlands

${ }^{6}$ Dept. of Pharmacy, Radboud University Nijmegen Medical Centre, Nijmegen, The Netherlands

To be submitted 


\section{ABSTRACT}

Many drugs undergo one or more variations in the aspect of drug quality after marketing approval. The application of each individual variation is often assessed and approved by drug regulatory authorities on an independent, case-by-case basis, unless multiple variations are submitted at once. Theoretically, post-marketing changes may have a cumulative effect on the quality of a medicinal product due to the numerous, nonconsecutive post-marketing variations in a quality aspect being made. For example, the consequence of such cumulative effects on any given generic drug may lead to changes in its quality, meaning that the current drug may be different from the one that demonstrated bioequivalence with the brand-name drug. In order to investigate whether quality variations have a cumulative effect on the interchangeability of generic drugs, we analyzed the effect of critical post-marketing quality variations for 10 active substances with a low solubility (BCS class II and IV). To this end, we developed a risk assessment model that combines all critical variations applied to brand-name or generic drugs.

According to the model, the totality of post-marketing variations would affect $17 \%$ of the drugs assessed ( $n=20$ out of 115), of which 13\% (15 out of 115) were generic drugs ( $n=99)$ and $4 \%$ (5 out of 115$)$ were brand-name drugs $(n=16)$. This showed that a limited number of generic drugs and brand-name drugs is affected by approved critical variations in a cumulative manner. Based on the model validation process, the model tended to overestimate the effect of post-marketing variations on the interchangeability of generic drugs. However, as the number of critical variations identified per drug was low ( $n=2$ in average), our concerns of frequent critical variations leading to significant changes in the long-time registered generic drugs can neither be relieved nor strengthened.

To conclude, among the investigated active substances, the cumulative effect of quality variations might affect the bioequivalence of a few generic drugs. Although regulatory action may not be necessary at the moment, the effect of critical variations on generic drugs should be re-assessed in the future, using an improved risk assessment model.

Key words: Bioequivalence, generic drug, interchangeability, quality variations, risk assessment 


\section{INTRODUCTION}

The interchangeability of generic drugs has always been a source of controversy among physicians and patients(1-3). Because brand-name drugs are often replaced by generic drugs, which are cheaper, the main source of controversy is whether generic drugs and brand-name drugs are indeed interchangeable. Many studies have investigated the interchangeability of generic drugs in general and some specific drugs in particular (2, 46), but conclusions are divergent. In a survey of 64 cardiology experts in the USA, 54 recurrences of arrhythmia as a result of generic substitution were reported, which triggers serious concerns regarding drug substitution in anti-arrhythmic therapy(2). On the other hand, according to a systematic review of seizure control (4) and a study of clozapine (5), treatment with generic drugs is not different from treatment with brandname drugs. Kesselhiem et al. reported that changes in pill color increase the risk of non-persistence in patients treated with antiepileptic drugs (6). This may have important clinical implications.

In drug regulation, one factor that may affect the interchangeability of generic drugs is post-marketing variations in the aspect of drug quality (i.e. so-called quality variation). When a drug is marketed, post-marketing quality variations (e.g., manufacturing process) are allowed, as long as the positive benefit-risk balance of the drug as demonstrated in registration studies is preserved. Applications for the so-called post-marketing quality variations for a drug are often submitted non-consecutively to regulatory authorities, and the impact on the benefit-risk balance of the drug is assessed on a caseby-case basis. For a major or critical variation that might affect the pharmacokinetics of the active substance, additional studies (e.g., dissolution studies and sometimes an in vivo bioequivalence study) may be requested to demonstrate the comparability of the drug before and after the changes. As a rule of thumb, the longer a drug is marketed, the more variations are applied.

It is conceivable that multiple, individually accepted critical variations made during the life-time of a drug may have a cumulative effect, changing the quality of a drug relative to the quality of the drug at the time of registration. For generic drugs, the change in quality may affect their bioequivalence relative to that of the brand-name drug, which raises questions about the interchangeability of generic drugs. Moreover, the cumulative effect of changes to the brand-name drug may also affect the interchangeability of generic drugs. However, regulatory authorities do not regularly assess the cumulative effect of post-marketing changes on the quality of drugs as a routine task.

Therefore, the objectives of this study were to develop a risk assessment model to quantify the cumulative effect of multiple quality variations on both generic and brandname drugs, and to estimate the potential risk of the variations affecting the interchangeability of generic drugs. 


\section{METHOD}

\section{Risk assessment model}

\section{The Model Concept}

The risk assessment model was based on the concept of risk ( $R$ ) being the multiplicative function of both hazard $(H)$ and exposure (E), i.e. $R=H \times E(7)$. In this study, hazard was defined by considering the characteristics of orally administered drugs with systemic exposure. The hazard score measures the sensitivity of a medicinal product to quality variations. In other words, the risk that the interchangeability of a generic drug is affected by quality variations is higher for drugs with a higher hazard than for drugs that have undergone the same quality variations but which have a lower hazard. In our model, we defined the hazard ratio (i.e., the absolute hazard divided by the minimum hazard) of drugs, with the minimum hazard value being considered the baseline level. Exposure was defined as the cumulative effect of the selected critical post-marketing quality variations made to the medicinal product from the date of licensing up to the date of the latest variation.

\section{Hazard identification and characterization}

To model drug hazard, five critical factors involving physicochemical parameters, production technology, and drug dosage form were identified and relative risk scores were given by the investigators. These factors were defined as follows.

\section{Lipophilicity (F1)}

The lipophilicity of a chemical compound is measured by log $\mathrm{P}$ and is critical for determining the pharmacokinetic properties of drug molecules (8). As reported by Leeson et al. (9), the relative risk score (2,3, or 4) was defined for three categories of drugs according to their log $P$ values (Table 1 ). 
Table 1. Definitions of relative risk scores for hazard and exposure components.

\begin{tabular}{|c|c|c|c|}
\hline Hazard component & \multicolumn{2}{|l|}{ Categories } & $\begin{array}{l}\text { Relative risk score } \\
\text { (1= no risk) }\end{array}$ \\
\hline \multirow[t]{3}{*}{$\log P$} & \multicolumn{2}{|c|}{ Ideal range: $1.56-3.34$} & 2 \\
\hline & \multicolumn{2}{|c|}{ 10\%-90\% percentile: $-0.65-1.56 ; 3.34-5.36$} & 3 \\
\hline & \multicolumn{2}{|c|}{ Outlier zone: $<-0.65$ or $>5.36$} & 4 \\
\hline \multirow[t]{2}{*}{ Therapeutic Index } & \multicolumn{2}{|c|}{ Non-narrow therapeutic range } & 2 \\
\hline & \multicolumn{2}{|c|}{ Narrow therapeutic range } & 4 \\
\hline \multirow[t]{5}{*}{ Dosage form } & \multicolumn{2}{|c|}{ Oral solutions } & 2 \\
\hline & \multicolumn{2}{|c|}{ Standard coating tablets or capsules } & 2.5 \\
\hline & \multicolumn{2}{|c|}{ Enteric-coated or delayed-release tablets or capsules } & 3 \\
\hline & \multicolumn{2}{|c|}{ Controlled or sustained-release capsules } & 3 \\
\hline & \multicolumn{2}{|c|}{ Controlled or sustained-release tablets } & 3.25 \\
\hline \multirow[t]{2}{*}{ Dose } & \multicolumn{2}{|l|}{$>10 \mathrm{mg}$} & 1.5 \\
\hline & \multicolumn{2}{|l|}{$\leq 10 \mathrm{mg}$} & 2.25 \\
\hline \multirow[t]{5}{*}{ Absorption dynamics } & \multirow{3}{*}{$\begin{array}{l}\text { Absorption } \\
\text { mechanisms }\end{array}$} & Passive diffusion & 1.5 \\
\hline & & Known active transport mechanism & 1.875 \\
\hline & & Known CYP $3 A 4$ or P-gp interaction & 1.875 \\
\hline & \multirow{2}{*}{$\begin{array}{l}\text { Delivery } \\
\text { conditions }\end{array}$} & Non site-specific absorption & 1.5 \\
\hline & & Site-specific absorption & 2.25 \\
\hline Exposure component & \multicolumn{2}{|c|}{ Critical variations } & $\begin{array}{l}\text { Relative risk score } \\
\text { (1= no risk) }\end{array}$ \\
\hline \multirow[t]{2}{*}{ Bioavailability-1 } & \multicolumn{2}{|l|}{ B.II.a.3 } & 18 \\
\hline & \multicolumn{2}{|l|}{ B.II.b.3 } & 16 \\
\hline \multirow[t]{3}{*}{ Bioavailability-2 } & \multicolumn{2}{|l|}{ B.II.b.4 } & 4 \\
\hline & \multicolumn{2}{|l|}{ B.Il.b.1 } & 3.8 \\
\hline & \multicolumn{2}{|l|}{ B.l.a.2 } & 3.6 \\
\hline \multirow[t]{2}{*}{ Bioavailability-3 } & \multicolumn{2}{|l|}{ B.II.d.1 } & 2.52 \\
\hline & \multicolumn{2}{|l|}{ B.I.a.1 } & 2.52 \\
\hline Bioavailability-4 & IB-33 (2006 & & 2 \\
\hline & B.II.a.4 & & 2 \\
\hline & B.II.a.2 & & 1.8 \\
\hline & B.Il.b.2 & & 1.6 \\
\hline & B.II.c.1 & & 1.4 \\
\hline & B.II.c.2 & & 1.4 \\
\hline
\end{tabular}

\section{Therapeutic Index (F2)}

For drugs with a narrow therapeutic index (NTI), small changes in blood concentration can lead to significant changes in the pharmacodynamic response $(10,11)$ and are thus expected to have a higher hazard than non-NTI drugs. Thus, the relative risk score for NTI drugs was defined as 4; it was 2 for non-NTI drugs (Table 1). 
In this study, the American Pharmacy Association definition of NTI drugs was used (12). An exhaustive list of NTI drugs was obtained by combining the list of NTI drugs available in the Scale-Up and Post-Approval Changes (SUPAC) guideline of the Food and Drug Administration (FDA) (13), with recommendations in the Medicine Substitution Guideline (Handleiding Geneesmiddelsubstitutie) produced by the Royal Association of Pharmacists of the Netherlands (14).

\section{Dosage Form Properties (F3)}

Guideline 2010/C17/01 of the European Commission(15), which provides guidance on the categories of post-marketing variations, makes a clear distinction between oral solutions and immediate-release solid oral dosage forms on the one hand and entericcoated and sustained-release dosage forms on the other. Five categories were defined with relative risks ranging from 2 to 3.25 (Table 1 ).

\section{Dose (F4)}

Drug dose varies, such that the active substances can comprise between $0.1 \%$ and $90 \%$ of the total mass of the dosage form (16). Medicinal products were divided into two groups (i.e., amount of active substance in the one formulation $>10 \mathrm{mg}$ and $\leq 10 \mathrm{mg}$ ), based upon consensus among the authors, and the risks were defined as 1.5 and 2.25, respectively (Table 1 ).

\section{Absorption Dynamics (F5)}

With regard to absorption dynamics, two aspects of mechanisms and delivery conditions were taken into account. Passive diffusion, active transport, and interaction with CYP3A4 or P-glycoprotein were assigned a relative risk of 1.5, 1.875 and 1.875, respectively. For delivery conditions, site-specific absorption was assigned a risk score 2.25, and non site-specific absorption a score of 1.5 (Table 1 ).

The hazard related to absorption dynamics (AD) was calculated as follows:

$$
A D=\left(\sum \text { absorption mechanism }\right) \times \text { delivery condition }
$$

Calculation of hazard ratio of a drug

The absolute hazard of a drug was calculated based on the properties of the drug in terms of the five factors as follows: $\mathrm{H}_{\mathrm{ab}}=(\mathrm{F} 1 \times \mathrm{F} 2 \times \mathrm{F} 3 \times \mathrm{F} 4 \times \mathrm{F} 5)$. As mentioned before, for any given drug product, a hazard ratio was calculated by dividing the absolute hazard by the minimum hazard, i.e. $\mathrm{H}=\mathrm{H}_{\mathrm{ab}} / \mathrm{H}_{\min }$. According to Table $1, \mathrm{H}_{\min }$ was 27 as a constant. 


\section{Exposure identification and characterization}

\section{Selection of critical variations}

According to guideline 2010/C17/01 of the European Commission(15), post-marketing quality variations can be classified as being minor (type IA), non-minor (type IB), or major/combined (type II). This implies that different variations have a different effect on the quality aspect of drugs. Type IA variations contain non-relevant and minor changes that are considered not likely to cause noticeable changes in the quality of medicinal products. For this reason, only the guideline sections covering type II (major) and type IB (non-minor) post-marketing variations that might affect drug quality and bioavailability were reviewed. The critical variations that may affect generic drugs were selected based on the evaluation of all type II and type IB variations (Table 2). The corresponding variations in the previous guidelines (versions of 1998 and 2006) were also checked, and one extra variation (i.e. IB-33 in the guideline 2006) was added (Table 2).

Table 2. The selection of critical quality variations based on EC guideline on post-marketing variations (2010).

\begin{tabular}{|c|c|}
\hline Variation Code & Variation Details \\
\hline B.I.a.1 & $\begin{array}{l}\text { Change in the manufacturer of a starting material/ reagent/ intermediate used in the } \\
\text { manufacturing process of the active substance or change in the manufacturer of the active } \\
\text { substance, where no Ph. Eur. Certificate of Suitability is part of the approved dossier. }\end{array}$ \\
\hline B.I.a.2 & Changes in the manufacturing process of the active substance. \\
\hline B.II.a.2 & Change in the shape or dimensions of the pharmaceutical form. \\
\hline B.II.a.3 & Changes in the composition (excipients) of the finished product. \\
\hline B.Il.a.4 & Change in coating weight of oral dosage forms or change in weight of capsule shells. \\
\hline B.Il.b.1 & $\begin{array}{l}\text { Replacement or addition of a manufacturing site for part or all of the manufacturing process } \\
\text { of the finished product. }\end{array}$ \\
\hline B.Il.b.2 & Change to batch release arrangements and quality control testing of the finished product. \\
\hline B.Il.b.3 & Change in the manufacturing process of the finished product. \\
\hline B.Il.b.4 & Change in the batch size (including batch size ranges) of the finished product. \\
\hline B.II.c.1 & Change in the specification parameters and/ or limits of an excipient. \\
\hline B.II.c.4 & $\begin{array}{l}\text { Change in synthesis or recovery of a non-pharmacopoeial excipient (when described in the } \\
\text { dossier) }\end{array}$ \\
\hline B.Il.d.1 & Change in the specification parameters and/ or limits of the finished product. \\
\hline IB-33 $(2006)^{*}$ & Minor change in the manufacture of the finished product. \\
\hline
\end{tabular}

* One discrepancy that did appear during extrapolation involved variation number IB 33 of the 2006 guideline, detailing a "Minor change in the manufacture of the finished product", to which variation B.II.b.3 of the 2010 guideline provided no equivalent alternative. This resulted in variation IB-33 being classified as a separate variation during the model development.

\section{Definition of the selected variations}

Each selected variation was given an individual numerical score, determined by the investigators. A high score indicates a high possibility that the variation will change the quality of the drug. The variations were divided into four groups, based on the possibility that drug bioavailability would be affected, and were also ordered within each group 
following the same rule, such that the relative risk was higher for "bioavailability 1 " than for "bioavailability 4". They were defined firstly for a key variation in each group, i.e., B.II.b.3 (score = 16), B.II.b.4 (score = 4, square root of 16), B.II.d.1 (score $=2.52$, cube root of 16) and B.II.a.4 (score $=2$, the quadruple root of 16), respectively. For the rest variations in the groups, the score was defined relatively higher or lower to the score of the key variation in the groups (Table 1).

\section{Coefficient Theta}

A coefficient theta $(\theta)$ was introduced to estimate the risk of repeating, for a given medicinal product, the same variation more than once. $\theta$ was assigned a high or a low value $\left(\theta_{H}\right.$ and $\left.\theta_{L}\right)$ between 1.0 and 2.0 for up to five repeat variations, i.e. $\theta_{H(1-5)}=(1.2$, $1.4,1.6,1.8,2.0)$ and $\theta_{\mathrm{L}(1-5)}=(1.1,1.2,1.3,1.4,1.5)$. The repeat variations considered when determining the value of $\theta$ mainly depended on the original assessment of the variation applications by regulatory authorities and on the content of the variations, as evaluated by the study investigators. The variation applications that raised no (or minor) questions about approval or for which detailed information was not found in the assessment record were assigned $\theta_{\mathrm{L}}$. The variation applications that raised major questions about approval were assigned $\theta_{\mathrm{H}}$.

\section{Calculation of Exposure to a Medicinal Product}

The exposure component of the model was calculated as a sum of risk scores of the critical variations $\left(E_{\mathrm{var}}\right)$ applied for a medicinal product, i.e.

$$
E_{\text {prod }}=\sum\left(E_{\text {var } 1}+E_{\text {var } 2}+\cdots E_{\text {varn }}\right)
$$

The coefficient $\theta$ was applied for calculating $E_{v a r}$ in the case of repeat variations, using the following formula:

$$
E_{\text {var }}=V^{n} \times\left(\theta_{0} \times \theta_{1} \times \cdots \theta_{n-1}\right)
$$

Where:

$\mathrm{V}$ : the relative risk score for the variation in Table 1;

$\mathrm{n}$ : the number of repeats of a variation $(\mathrm{n} \geq 1)$;

$\theta_{0}=1$

\section{Algorithm of the risk assessment model}

Based on the definitions for the model components, the total risk score of a medicinal product was calculated as below:

$$
\begin{gathered}
R=H \times E \\
R=\left(\frac{H_{a b}}{H_{\text {min }}}\right) \times \sum\left(E_{\text {var } 1}+E_{\text {var } 2}+\cdots E_{\text {varn }}\right)
\end{gathered}
$$




\section{$\left.\sum \mathbb{Z}\left[\left[V_{1} \rrbracket^{n} \times\left(\theta_{0} \times \cdots \theta_{m-1}\right)\right]+\mathbb{Z}\left[V_{2} \rrbracket^{n} \times\left(\theta_{0} \times \cdots \theta_{n-1}\right)\right]+\cdots\left[V_{n} \rrbracket^{n} \times\left(\theta_{0} \times \cdots \theta_{m-1}\right)\right]\right]\right]$}

\section{Risk characterization of generic drugs}

\section{Determination of the threshold}

A threshold for the estimated risk was defined based on positive control cases. Eight medicinal products for which a clear risk of interchangeability of generic drugs had been identified during the quality variation application process were selected, based upon the experience and opinion of quality and pharmacokinetic assessors of the Medicines Evaluation Board (MEB) in the Netherlands. The assessors requested the redemonstration of bioequivalence via additional pharmacokinetic studies. The active substances of these medicinal products are bisoprolol, carbidopa / levodopa, ethionamide, acetylsalicylic acid, raloxifene, triameterene / hydrochlorothiazide, spironolactone, and hydrocholorothiazide.

The post-marketing variation profiles of these control cases were evaluated, and the total risk score was calculated for each case, using the model. The lowest risk score was used as threshold for the risk that post-marketing variations would affect the interchangeability of generic drugs .

\section{Dataset: selection of medicinal products}

To evaluate the cumulative effect of multiple quality variations on both generic and the brand-name drugs, we selected 10 active substances from Biopharmaceutical Classification System (BCS) classes II and IV (i.e., poor solubility) drugs. The rationale for selecting BCS class II and IV drugs is that poorly soluble drugs are sensitive to quality variations in terms of changes in bioavailability $(17,18)$. The literature was used to identify BCS class II and IV drugs(19). Eighty-one drugs (i.e., active substances) identified as BCS class II and IV by Benet et al. were available at the MEB database. We selected those active substances with the highest number of generic drugs registered in the Netherlands, i.e. naproxen, losartan, olanzapine, citalopram, bicalutamide, lamotrigine, domperidone, carbamazepine, mesalazine, and loratadine.

Drugs in different strengths were considered as one drug, because in most cases, tablets/capsules containing different amounts of the same drug are manufactured in the same way. Therefore, a change introduced by quality variation would apply to all strengths of the drug. All completed? applications for post-marketing variations for the selected drugs were collected from the MEB internal database. For brand-name drugs, variations were collected for the period starting 2 years before registration of the first generic drug, in order to avoid overestimation and to cover roughly the same time frame as for generic drugs. 


\section{Model validation}

To validate the model, 16 medicinal products were randomly selected from two categories (8 per category), i.e., medicinal products with an estimated total risk higher or lower than the threshold. The 8 positive control cases used for identifying the threshold were also included, making 24 medicinal products. A file summarizing all selected critical quality variations (Table 1) was prepared for each selected medicinal product, with blinding of the drug name, company name, registration number, and the estimated results. The 24 files were sent in mixed order to three voluntarily participating pharmacokinetic assessors at the MEB, who were asked to provide their opinion on whether a new bioequivalence study would be necessary to re-ascertain bioequivalence of the modified product, considering all selected critical variations in combination. The consistency between the results predicted by the model and the assessors' opinion on these 24 cases was taken to reflect model validation.

\section{RESULTS}

Of the identified poorly soluble drugs, the 10 active substances that had the most generic drugs registered in the Netherlands are listed in Table 3. A total of 151 generic drugs were assessed for critical quality variations. Of these drugs, 52 generic drugs did not have any critical variations and were therefore excluded from this study. Sixteen brand-name drugs that contained one of the active substances were identified. In total, 115 drugs (99 generics and 16 brand-name) were investigated for the cumulative effects of critical quality variations. 


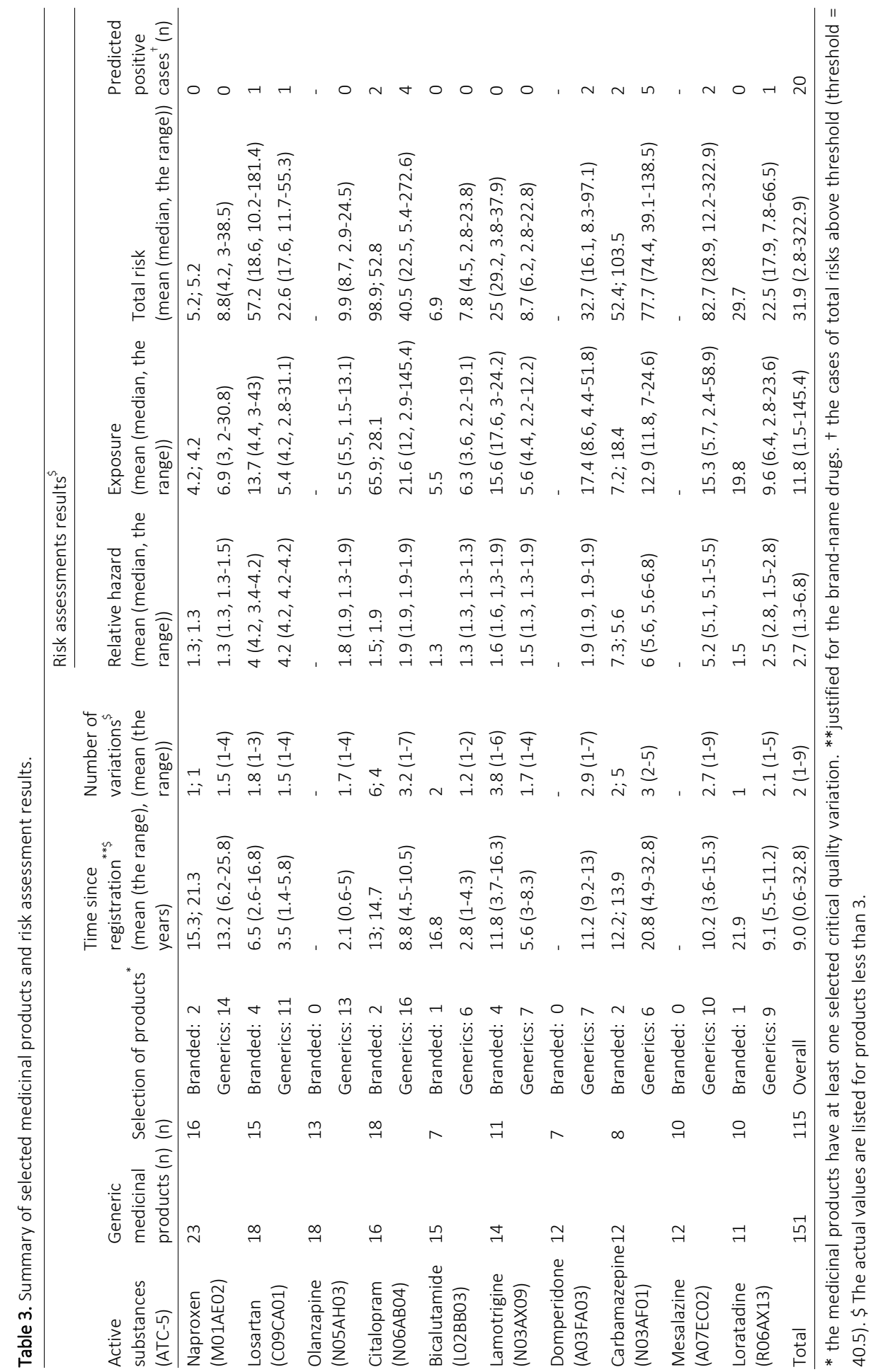


The average post-registration lifetime of the drugs was 9 years (range 0.6-32.8 years). On average, two critical variations had been processed for each drug (range 1-9 variations). There was no correlation between post-registration drug lifetime and number of critical variations $\left(r^{2}=0.1\right)$. The average drug lifetime for the brand-name drugs (adjusted as described above based on the registration date of the first generic drug) was longer (12.7 years) than that of the generic drugs (8.4 years) (Table 4 ). The average number of critical variations identified was 3 (range 1-6) for the brand-name drugs and 2 (range 1-9) for the generic drugs (Table 4).

Table 4. Evaluation of risk assessment results for the brand-name and generic drugs.

\begin{tabular}{lllll}
\hline & $\begin{array}{l}\text { Life time } \\
\text { (mean (the range), } \\
\text { years) }\end{array}$ & $\begin{array}{l}\text { Selection of variations } \\
\text { (mean (the range)) }\end{array}$ & $\begin{array}{l}\text { Predicted positive } \\
\text { cases* }\end{array}$ & $\begin{array}{l}\text { Predicted negative } \\
\text { cases** }\end{array}$ \\
\hline $\begin{array}{l}\text { Brand-name drugs } \\
(\mathrm{n}=16)\end{array}$ & $12.7(2.6-21.9)$ & $2.8(1-6)$ & $5(31.3 \%)$ & $11(68.7 \%)$ \\
$\begin{array}{l}\text { Generic drugs ( }=99) \\
\text { Total }(\mathrm{n}=115)\end{array}$ & $8.4(0.6-32.8)$ & $2.2(1-9)$ & $15(15.2 \%)$ & $84(84.8 \%)$ \\
$\begin{array}{l}\text { Predicted positive } \\
\text { cases* }(n=20)\end{array}$ & $14.7(3-32.8)$ & - & $20(17.4 \%)$ & $95(82.6 \%)$ \\
$\begin{array}{l}\text { Predicted negative } \\
\text { cases** }(n=95)\end{array}$ & $7.8(0.6-25.8)$ & $2(1-6)$ & - & - \\
\hline
\end{tabular}

* the drugs of total risks above threshold (threshold $=40.5$ ); ${ }^{* *}$ the drugs of total risks below threshold (threshold $=40.5$ ).

\section{Risk assessment}

The calculated relative hazard for all drugs was in the range 1.3-6.8, and exposure was in the range 1.5-145.4 (Table 3). The mean total risk that drug quality would be affected by critical variations was 31.9 (range $2.8-322.9$ ). The estimated total risk of drugs was not correlated with the drug lifetime $\left(r^{2}=0.1\right)$.

The minimum total risk threshold, calculated for the 8 positive control cases, was 40.5 (range 40.5-223.3). This means that when the total risk for a medicinal product is above the threshold, its quality is potentially different from that at the moment of registration, and therefore re-demonstration of bioequivalence should be requested. Twenty drugs (out of 115) had a total risk higher than the threshold (5 brand-name drugs and 15 generic drugs) (see Table 3). The drugs most frequently predicted to have a total risk higher than the threshold contained carbamazepine (7 out of 8 drugs).

The average drug lifetime and the number of critical variations were higher with the above-threshold drugs than for the below-threshold drugs (14.7 vs. 7.8 years, and 4.6 vs. 2 variations, respectively) (Table 4). 


\section{Validation}

The assessors were in agreement with the model regarding the 8 predicted negative cases, but not for the 8 predicted positive cases. The assessors also considered that new demonstration of bioequivalence would be required for 1 of the 8 predicted positive cases. All 8 positive control cases were considered "positive" by all assessors.

\section{DISCUSSION}

In this study, the model predicted that, for most of the drugs investigated $(83 \%, n=95$ out of 115), the cumulative effect of critical variations would not lead to a request for the re-demonstration of bioequivalence between the generic and the brand-name drugs. Fifty-two generic drugs were excluded from the study because there had been no critical variations. Taking these cases into account, in only $12 \%$ (20 out of 167 ) of the generic drugs containing the 10 active substances investigated was there a risk that multiple post-marketing variations affected the bioequivalence demonstrated in the registration of the generic drugs.

The active substances investigated are particularly sensitive to quality variations, being poorly soluble (BCS class II or IV drugs), and have an oral solid dosage form. Moreover, the brand-name drugs containing these active substances have given rise to a substantial number of generic drugs that have been registered for long enough to have undergone a number of variations. Therefore, the effect of these post-marketing changes may represent the 'worst case' scenario regarding the cumulative effect of critical variations. On the basis of our findings, the proportion of generic drugs with a significant risk that cumulative critical variations would necessitate re-assessment of bioequivalence is not expected to be higher than the $12 \%$.

The period over which the drugs were investigated was relatively long, with a maximum of 32.8 years ( 9 years in average). There was a mean of 2 variations (range $1-9$ ) per drug and the length of the investigation period was not correlated with the number of critical post-marketing variations. Thus at the present time, most generic drugs would appear to be similar to that when the drug was originally approved.

\section{Risk assessment model}

The basic concept of risk assessment introduced in the book of "Risk Assessment in the Federal Government: Managing the Process" (7) was followed to build the model. In this book, risk assessment meant "the characterization of the potential adverse health effects of human exposure to environmental hazards". The main four steps are hazard identification, dose-response assessment, exposure assessment, and risk characteriza- 
tion. For the purpose of our study, we adapted the steps in the risk assessment into drug hazard and exposure to critical variations.

\section{Drug hazard}

We assessed five properties of drugs (i.e., lipophilicity, the Therapeutic Index, dosage form properties, dose, and absorption dynamics) as being potentially sensitive to critical variations. Log P, a measure of lipophilicity, influences drug transport and target binding $(8,9)$. As biological activity increases with increasing lipophilicity, log P of a drug must not be too low. However, if $\log \mathrm{P}$ is too high, there is a greater probability that the compound will bind to hydrophobic protein targets other than the desired one, potentially causing toxicity. The formulation of drugs with a high or low lipophilicity is often adapted to ensure optimal bioavailability. Therefore, we considered that drugs with an extreme log $P$ value (e.g., <1.56, and > 3.34) would be more susceptible to the effect of critical variations than those drugs with a log $P$ value in the normal range (1.56-3.34) (9).

Most drugs have a wide therapeutic window, meaning that the difference between therapeutic and toxic concentrations is relatively large. In the clinic, drug monitoring during treatment is usually not needed. In contrast, NTI drugs have a small difference between therapeutic and toxic concentrations, which can give rise to drug-related problems in terms of non-optimal dose, drug interaction, and need for drug concentration monitoring (10). Therefore, NTI drugs are expected to be more sensitive to critical variations than other drugs (i.e., to have a higher risk score).

Dosage forms were categorized mainly in terms of immediate release and modified release formulations. The risk score of an immediate release drug is expected to be lower than that of a modified release drug. In the quality guideline (European Commission 2010)(15), for example, the change in coating weight of an immediate-release oral tablet or capsule is defined as a minor (type IA) variation, whereas that made to a gastro-resistant or modified-release tablet or capsule is classified as a major (type II) variation. Furthermore, considering drug delivery technology, manufacturing process difficulties, and physical components of the different dosage forms (16), enteric-coated or delayed-release tablets/capsules were given a higher risk score than standard coated tablets/capsules. We also distinguished between controlled-/sustained-release tablets and capsules, based upon the concept of manufacturing difficulties. We assumed that controlled or sustained release tablets need more technological and procedural considerations than standard tablets/capsules or controlled or sustained release capsules (16). For tablets, drug release is governed by the constituents of the drug and the coating powder (i.e., there is no functional separation between the coating and active moieties), and the manufacturing technique has to ensure that a homogenous compressed powder is produced(16). In contrast, drug release from capsules is governed by the external coating of the capsule, which is a separate entity that surrounds an independent powder mixture. Therefore, we assumed that variations applied to capsule formula- 
tions would have a smaller effect on bioavailability, and thus a lower risk score, than the same variations applied to controlled- and sustained-release tablets.

The risk score was considered relative to the dose, as the total weight of a tablet/capsule can be varied. As the quantity of active substance decreases, it becomes technologically more complex to produce a tablet or solution (16). Therefore, assuming that post-marketing variations might affect drug dissolution or bioavailability, the lower the dose of active substance initially present, the higher the risk that the bioavailability of the active substance will change. Consequently, based on the experience and expertise of the investigators, a cut-off value of $10 \mathrm{mg}$ was used, with higher risk scores being assigned to drug formulations containing doses lower than $10 \mathrm{mg}$.

For all oral dosage forms, drug absorption and permeation are critical steps after drug dissolution (18). The mechanisms of drug absorption are varied, ranging from passive paracellular transport to specialized active influx (16). For absorption dynamics, the more complex/specific the absorption process is, the more likely that bioavailability will be influenced by changes to the drug formulation. Therefore, drugs with more complex absorption processes were given a higher risk score in the model - drugs absorbed via passive diffusion were given a lower risk score than drugs absorbed by active transport. Moreover, drugs that dissolve in the gastrointestinal tract may interact with cytochrome p-450 (CYP-450) enzymes or efflux proteins such as P-glycoprotein. The bioavailability of active substances that interact with CYP-3A4 enzymes (which have the highest concentration in the intestinal mucosa) (20) and/or P-glycoprotein may decrease as a result of metabolism to inactive metabolites prior to absorption or drug efflux antagonizing the absorption process(16). Therefore, drugs that interact with CYP3A4 or P-glycoprotein were assigned a risk score similar to that of drugs that undergo active transport. In addition, as some formulations may be absorbed in a specific section of the gastrointestinal tract (21), drugs with site-specific absorption were also assigned high risk scores in the model.

It is difficult to determine the absolute risk that the quality of a drug will change after critical variations because in principle every drug is at risk if a critical variation is applied. Thus in our model, a hazard ratio was calculated for each drug. The minimum hazard assumes that cumulative, critical variations to even the least risk-liable dosage form can theoretically change the drug's bioavailability or pharmacokinetic profile.

\section{Exposure to variations}

We classified critical variations into four groups based on the predefined impact of variations on drug bioavailability. The score for each variation was built on a system of exponential segregation, such that the highest variation score of bioavailability-4 group is the quadruple root of the lowest bioavailability-1 group score. This implies that a variation in the bioavailability-4 group must be repeated a minimum of four times in the lifetime of a single drug, to have the same predicted effect on drug quality as one variation in the bioavailability-1 group. 
We introduced the theta coefficient to model the risk of repeated variations, because repeated variations are considered to increase risk more than the sum of two independent variations. For example, add a new manufacturing site of a drug is considered as a variation, where the new site can be already part of the approved pharmaceutical manufacturing group registered at the regulatory authorities. If it is not considered part of the registered manufacturing group, then additional validation data must be provided and different conditions apply for the applicant. We therefore gave theta two possible values, either high $\left(\theta_{H}\right)$ or low $\left(\theta_{L}\right)$, depending on the magnitude of the effect of repeat post-marketing variations (e.g. addition of new manufacturing site) on drug quality and on the assessment of the application for that variation (i.e., whether major questions were raised by assessors).

Our study had a number of limitations. The value given to the theta coefficient $\left(\theta_{H}\right.$ and $\theta_{\mathrm{L}}$ ) was based on subjective judgment. Inter-assessor differences in theta coefficient may affect the risk estimated by the model that post-marketing variations will affect drug quality, and this is especially the case for drugs with a relatively high risk. Second, model outcomes may have been limited by the available data - the low percentage of predicted positive cases $(17 \%)$ may have been influenced by the small number of critical variations identified. Thus, we failed to demonstrate whether critical variations have a cumulative impact on the quality of generic drugs. It is also difficult to validate the model further with the current dataset because too few drugs have undergone multiple critical variations (for example, at least 5 critical variations in average). One option would be to select only those drugs that have undergone at least five critical variations. Furthermore, as this study only investigated drugs with an oral solid dosage form, the results cannot be simply extrapolated to drugs with other dosage forms, such as lozenges, formulations for injections/infusions, suppository etc.

The model probably overestimated the number of drugs that would require further proof of bioequivalence, as only 1 of the 8 model-predicted positive cases was considered "truly positive" by the experts. A possible reason for this is the incorrect weighting given to the factors of drug hazard. For example, the hazard ratios for carbamazepine generic drugs were in the range 5.6-7.3, which would prompt bioequivalence reassessment. We found that the predicted positive cases had a longer mean product lifetime and had undergone more variations than the predicted negative cases (Table 4). We subsequently established that the total risk score of drugs increased more than proportionally to the number of variations. Thus the model algorithm ascribed multiple variations a higher risk score than the sum of the individual variations. In addition, the use of a constant threshold that does not take into consideration the number of critical variations may lead to the situation by which more variations may increase the risk that drug quality is affected. The model requires further refinement.

To our knowledge, this is the first model to evaluate the outcome of cumulative effects of post-marketing variations on brand-name and generic drugs. The cumulative effect of the variations was investigated in 115 orally administered BCS class II and IV 
drugs registered for 0.6-32 year. On the basis of our model results, drug quality may have changed in $17 \%$ of the drugs, leading to a difference in bioavailability compared with that of the initial product at registration. Therefore, a new bioequivalence study should in principle be requested to re-demonstrate the similarity between the current generic drugs and the brand-name drug. In reality, however, the proportion of drugs that need to be re-assessed is expected to be even lower because the selection of drugs represents the worst-case scenario and our model overestimates the influence of quality variations. Because there had only been a limited number of quality variations for the drugs selected, we cannot conclude or refute that multiple critical variations significantly change the bioequivalence of generic drugs demonstrated at the moment of registration. Nevertheless, as the number of critical variations is expected to increase during a drug's lifetime, the model should be improved and used at a later stage to monitor the impact of post-marketing quality variations on generic drugs. 


\section{REFERENCES}

(1) Hauck, W.W. \& Anderson, S. Measuring switchability and prescribability: when is average bioequivalence sufficient? Journal of pharmacokinetics and biopharmaceutics22, 551-64 (1994).

(2) Reiffel, J.A. \& Kowey, P.R. Generic antiarrhythmics are not therapeutically equivalent for the treatment of tachyarrhythmias. The American journal of cardiology85, 1151-3, A10 (2000).

(3) Johnston, A., Stafylas, P. \& Stergiou, G.S. Effectiveness, safety and cost of drug substitution in hypertension. Br J Clin Pharmacol70, 320-34 (2010).

(4) Kesselheim, A.S. et al. Seizure outcomes following the use of generic versus brand-name antiepileptic drugs: a systematic review and meta-analysis. Drugs70, 605-21 (2010).

(5) Oluboka, O., Stewart, S., Landry, S. \& Adams, S. Does therapeutic equivalence follow bioequivalence? A randomized trial to assess clinical effects after switching from Clozaril to generic clozapine (genclozapine). Journal of clinical pharmacology50, 531-5 (2010).

(6) Kesselheim, A.S. et al. Variations in pill appearance of antiepileptic drugs and the risk of nonadherence. JAMA internal medicine173, 202-8 (2013).

(7) In: Risk Assessment in the Federal Government: Managing the Process (Washington (DC), 1983).

(8) van de Waterbeemd, H., Smith, D.A. \& Jones, B.C. Lipophilicity in PK design: methyl, ethyl, futile. Journal of computer-aided molecular design15, 273-86 (2001).

(9) Leeson, P.D. \& Davis, A.M. Time-related differences in the physical property profiles of oral drugs. Journal of medicinal chemistry47, 6338-48 (2004).

(10) Blix, H.S., Viktil, K.K., Moger, T.A. \& Reikvam, A. Drugs with narrow therapeutic index as indicators in the risk management of hospitalised patients. Pharmacy practice8, 50-5 (2010).

(11) Burns, M. Management of narrow therapeutic index drugs. Journal of thrombosis and thrombolysis7, 137-43 (1999).

(12) Manolakis, P.G. Prescription drug product substitution decision support. Journal of the American Pharmacists Association : JAPhA47, 328-38 (2007).

(13) Guidance for Industry; Immediate Release Solid Oral Dosage Forms Scale-Up and Postapproval Changes: Chemistry, Manufacturing, and Controls, In Vitro Dissolution Testing, and In Vivo Bioequivalence Documentation. (ed. Research, C.f.D.E.a.) (U.S. Food and Drug Administration (FDA), 1995).

(14) Handleiding Geneesmiddelsubstitutie. <https://www.knmp.nl/patientenzorg/geneesmiddelen/handleidinggeneesmiddelsubstitutie> (2012). 2012.

(15) Communication from the Commission - Guideline on the details of the various categories of variations to the terms of marketing authorisations for medicinal products for human use and veterinary medicinal products (2010/C 17/01). Official Journal of the European Union, (2010).

(16) Modern Pharmaceutics: Book 1, (Informa Healthcare: New York, 2009).

(17) Wu, C.Y. \& Benet, L.Z. Predicting drug disposition via application of BCS: transport/absorption/ elimination interplay and development of a biopharmaceutics drug disposition classification system. Pharmaceutical research22, 11-23 (2005).

(18) Grassi, M. Understanding Drug Release and Absorption Mechanisms: A Physical and Mathematical Approach CRC (Hoboken: 2007).

(19) Benet, L.Z., Broccatelli, F. \& Oprea, T.I. BDDCS applied to over 900 drugs. The AAPS journa/13, 519-47 (2011).

(20) Bambal, R.C., S. Evaluation of Drug Candidates for Preclinical Development: Pharmacokinetics, Metabolism, Pharmaceutics and Toxicology (Hoboken: 2010).

(21) Pithavala, Y.K., Heizer, W.D., Parr, A.F., O'Connor-Semmes, R.L. \& Brouwer, K.L. Use of the InteliSite capsule to study ranitidine absorption from various sites within the human intestinal tract. Pharmaceutical research15, 1869-75 (1998). 
Cumulative impact of post-marketing variations on interchangeability of generic drugs

Appendix I The calculations for identification of threshold (the eight positive control cases)

\begin{tabular}{|c|c|c|c|c|c|}
\hline \multirow[b]{2}{*}{ Active substances (ATC-5) } & \multirow[b]{2}{*}{$\begin{array}{l}\text { Life time } \\
\text { (years) }\end{array}$} & \multirow[b]{2}{*}{$\begin{array}{l}\text { Number of } \\
\text { variations }\end{array}$} & \multicolumn{3}{|c|}{ Risk assessment results } \\
\hline & & & $\begin{array}{l}\text { Relative } \\
\text { hazard }\end{array}$ & Exposure & Total risk \\
\hline Bisoprolol (C07AB07) & 11.3 & 5 & 4.2 & 52.9 & 223.3 \\
\hline Levodopa / carbidopa (N04BA02) & 21.7 & 7 & 3.3 & 27.4 & 88.9 \\
\hline Ethionamide (J04AD03) & 0.5 & 8 & 1.9 & 59.4 & 111.4 \\
\hline Acetylsalicylic acid (B01AC06) & 2.3 & 1 & 1.9 & 21.6 & 40.5 \\
\hline Raloxifene (G03XC01) & 0.5 & 2 & 1.9 & 24.6 & 46.2 \\
\hline $\begin{array}{l}\text { Triamterene / hydrochlorothiazide } \\
\text { (C03DB06 / C03AA03) }\end{array}$ & 36.3 & 5 & 1.9 & 50.5 & 94.8 \\
\hline Spironolactone (C03DA01) & 13.3 & 4 & 1.9 & 30.8 & 57.7 \\
\hline Hydrochlorothiazide (CO3AA03) & 9.3 & 6 & 1.9 & 35.8 & 67.1 \\
\hline The minimum total risk (the threshold) & & & & & 40.5 \\
\hline
\end{tabular}





\section{Chapter}

\section{Interchangeability of gabapentin generic formulations in the Netherlands: a comparative bioavailability study}

$\underline{\text { Yang Yu }}{ }^{1,2}$, Steven Teerenstra ${ }^{2,3}$, Floris Vanmolkot ${ }^{4}$, Cees Neef ${ }^{1,2}$, David Burger ${ }^{5}$ and Marc Maliepaard ${ }^{2}$

${ }^{1}$ Dept. Pharmacology, Maastricht University Medical Centre, Maastricht, The Netherlands

${ }^{2}$ Medicines Evaluation Board, Utrecht, The Netherlands

${ }^{3}$ Dept of Epidemiology, Biostatistics, and HTA, Radboud University Nijmegen Medical Centre, Nijmegen, The Netherlands

${ }^{4}$ Dept. Internal Medicine, Maastricht University Medical Centre, Maastricht, The Netherlands

${ }^{5}$ Dept. of Pharmacy, Radboud University Nijmegen Medical Centre, Nijmegen, The Netherlands

Clin Pharmacol Ther 94, 519-24 (2013) 


\section{ABSTRACT}

To investigate so-called "drift" with generic-generic drug substitution, a single dose, four-way crossover comparative bioavailability study was performed involving 24 healthy subjects and three generic and one branded formulation of a tablet containing $800 \mathrm{mg}$ gabapentin as test medication. The results showed that the $90 \%$ confidence intervals $(\mathrm{Cls})$ for the area under the drug concentration versus time curve $\left(\mathrm{AUC}_{0-\mathrm{t}}\right)$ and for the peak drug concentration $\left(C_{\max }\right)$ were within the acceptance range of $80-125 \%$ for all comparisons. The safety profiles of the different gabapentin formulations were comparable. To conclude, all three generic formulations of gabapentin were found to be bioequivalent with the branded formulation and with each other, indicating that the formulations are interchangeable. These results strongly indicate the absence of "drift" with gabapentin generic-generic substitution.

Keywords: bioequivalence, generic, gabapentin, substitution, interchangeability, pharmacokinetics, regulatory science. 


\section{INTRODUCTION}

After the patent of a branded medicine has expired, generic formulations become available as alternatives, but some patients and doctors are concerned about their use in daily clinical practice(1-3). For example, several studies have reported an increased risk of seizures, high switching-back rate, and frequent visits to the emergency unit when patients with epilepsy are switched from a branded medicine to a generic formulation(1, 2, 4, 5). Patients and prescribers are often reluctant to switch from innovator to generic drugs or from generic to generic drugs(5-9). From the regulatory authorities' perspective, the bioequivalence requirements are strict, so that generic drugs can be considered therapeutically equivalent to the branded drug $(10,11)$. In an evaluation of registered generic drugs, the Food and Drug Administration (FDA) in the United States reviewed 2070 bioequivalence studies from 1996-2007 and found the average difference in the area under the plasma drug concentration versus time curve (AUC) between generic and innovator drugs to be less than $5 \%$ (11). This means that clinical pharmacokinetic differences are unlikely to be the cause of adverse events when patients switch from an innovator drug to a generic version.

The FDA and European Medicine Agency (EMA) have been questioned about their guidance for generic medicinal products $(12,13)$, because according to these guidances, generic drugs are only compared with innovator drugs in single-dose bioequivalence studies applying the $80-125 \%$ criterion and not with other registered generic versions. Almost none of the publications reporting problems in generic drug substitution provide data on actual drug exposure, which makes it difficult to ascertain whether these and other concerns about the presumed higher risk of treatment failure with generic products are valid(4, 6, 10, 14, 15). Theoretically, there may be so-called "drift" when one generic drug is exchanged for another $(1,16)$, such that although both generic drugs show bioequivalence with the innovator drug, they do not necessarily show bioequivalence with each other (the $90 \%$ confident interval (Cl) for this comparison may not obey the $80-125 \%$ criterion). However, based on the results of the retrospective study from the FDA, the mean ( \pm S.D.) of the geometric mean ratio (generic:innovator drug) was $1.00 \pm 0.06$ for the drug peak plasma concentration $\left(C_{\max }\right)$ and $1.00 \pm 0.04$ for the AUC, when all tested generics were considered (11). With a point estimate so close to 1.0 , it is unlikely that there is "drift" among registered generic formulations. Indeed, although several articles postulated the possibility of such "drift" with generic drug use, very few studies have investigated this potential effect(1, 16-18), and those that did, did not provide clinical bioavailability data for different generic drugs.

In the Netherlands, many generic versions of innovator drugs are available, and generic-generic exchange is common in clinical practice. Although testing the comparable bioavailability of different generic drugs is not required for the registration of a generic medicinal product, the Dutch regulatory authority thought it prudent to investigate the consequences of this policy for currently marketed generic formulations. In this light, in 
2011, we performed a simulation study to address the potential "drift" among topiramate and gabapentin generic products(17). This simulation study used exposure data for the different generic drugs obtained from drug registration files archived at the Dutch Medicine Evaluation Board. Inter-study comparison did not detect "drift" for gabapentin generics. However, this simulation study was limited by its retrospective inter-study (i.e. not intra-study) design, and by the fact that currently marketed drugs (batches) were not compared but instead those used for the registration. In order to address these issues, we conducted the current clinical bioequivalence study comparing gabapentin exposure after administration of the gabapentin branded formulation (Neurontin ${ }^{\circ}$ ) and three generic formulations currently marketed in the Netherlands. This four-way crossover design allows a more robust assessment of the potential "drift" during generic-generic exchange for gabapentin.

\section{RESULTS}

The clinical phase of this study took place in October and November 2011. Twenty-four healthy volunteers (14 females, 10 males) were enrolled and completed the study. Their mean age was 35 (range 21-55) years, and the mean body mass index (BMI) was 23.6 $\mathrm{kg} / \mathrm{m}^{2}$ (range 19.9-30.0 kg/m²). Gabapentin levels were measured in all plasma samples (no samples were missing). Levels of gabapentin in samples collected before drug administration were well below $5 \%$ of the Cmax, indicating that there was no relevant carry-over concentration between the four drug administration periods.

\section{Pharmacokinetics}

The mean plasma concentration-time curves for gabapentin by drug formulation are shown in Figure 1, and the pharmacokinetic parameters of each drug are summarized in Table 1. The elimination half-life $\left(t_{1 / 2}\right)$ of the different gabapentin formulations ranged from 7 to 8 hours. The mean $C_{\max }$ for the different formulations ranged between 5.3 and $5.6 \mathrm{mg} / \mathrm{L}$, and the mean AUC ranged from 58 to $60 \mathrm{~h} \cdot \mathrm{mg} / \mathrm{L}$. The mean residual area was less than $3 \%$ for all formulations, indicating that it was sufficient to measure gabapentin concentrations over 48 hours. 

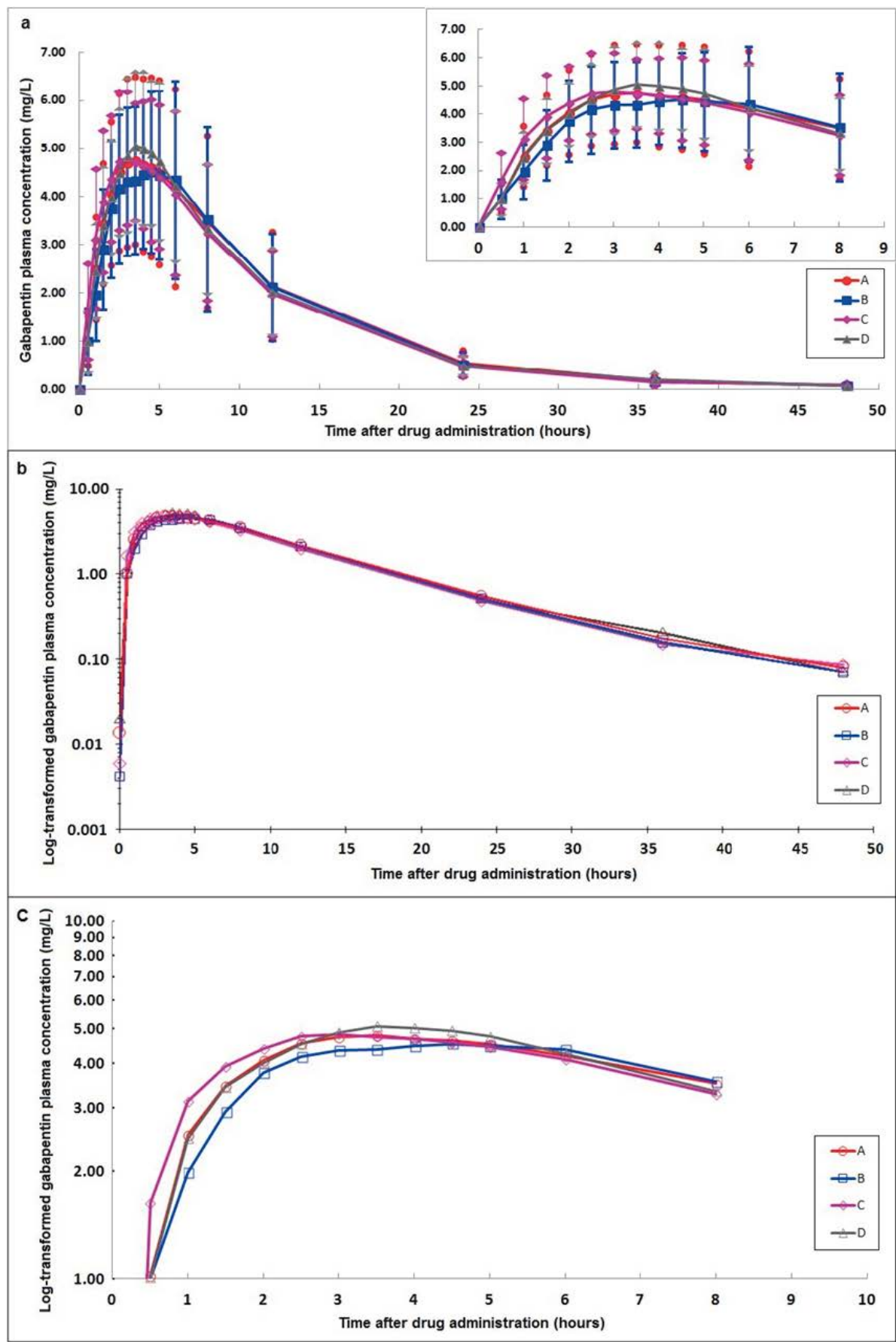

Figure 1. Mean gabapentin plasma concentration-time curves for innovator and generic formulations of gabapentin $(n=24)$. (a) Mean gabapentin plasma concentration-time curves \pm SD for innovator and generic formulations of gabapentin $(n=24)$. ' $A$ ' represents the mean of gabapentin plasma concentration ( $Y$-axis) at 


\section{Chapter 5}

every sampling time (X-axis) among the 24 subjects with treatment of Neurontin (innovator); ' $B$ ', ' $C$ ' and ' $D$ ' represent for the three generic formulation of gabapentin. The mean pre-dose level for each formulation is indicated at Time point $=0$. A detail plot shows the data up to 8 hours after administration (b) Mean gabapentin plasma concentration-time curves for innovator and generic formulations of gabapentin $(n=24)$ in a semilogarithmic plot. (c) Detail plot showing the mean gabapentin plasma concentration-time curves for innovator and generic formulations up to 8 hours after administration and plasma concentrations higher than $1 \mathrm{mg} / \mathrm{L}$ in a semi-logarithmic plot.

Table 1. Summary of pharmacokinetic variables ( $n=24$ subjects).

\begin{tabular}{|c|c|c|c|c|c|c|c|c|}
\hline \multirow[t]{2}{*}{ Parameters } & \multicolumn{2}{|c|}{ Treatment A } & \multicolumn{2}{|l|}{ Treatment B } & \multicolumn{2}{|l|}{ Treatment C } & \multicolumn{2}{|l|}{ Treatment D } \\
\hline & mean $\pm S D$ & median & mean $\pm S D$ & median & mean $\pm S D$ & median & mean $\pm S D$ & median \\
\hline $\mathrm{C}_{\max }(\mathrm{mg} / \mathrm{L})$ & $5.33 \pm 1.79$ & 5.42 & $5.43 \pm 1.86$ & 5.49 & $5.48 \pm 1.44$ & 5.46 & $5.62 \pm 1.65$ & 5.82 \\
\hline$A \cup C_{0-t}\left(h^{*} m g / L\right)$ & $60.4 \pm 24.1$ & 54.6 & $58.5 \pm 23.0$ & 58.3 & $58.3 \pm 19.6$ & 57.7 & $59.0 \pm 21.5$ & 61.5 \\
\hline$A \cup C_{\text {inf }}\left(h^{*} m g / L\right)$ & $61.4 \pm 24.0$ & 55.2 & $59.3 \pm 23.0$ & 58.9 & $59.5 \pm 19.7$ & 59.4 & $60.0 \pm 21.7$ & 62.3 \\
\hline Residual area (\%) & $2.03 \pm 2.08$ & 1.14 & $1.61 \pm 1.54$ & 1.25 & $2.04 \pm 1.47$ & 1.70 & $1.66 \pm 1.65$ & 1.12 \\
\hline$T_{\max }(h)^{a}$ & $(2.50-8.00)$ & 4.00 & $(2.00-8.00)$ & 4.25 & $(1.50-6.00)$ & 3.50 & $(1.50-8.00)$ & 3.75 \\
\hline$t_{1 / 2}(h)$ & $7.97 \pm 2.63$ & 7.28 & $7.68 \pm 2.04$ & 7.28 & $8.32 \pm 2.89$ & 7.58 & $7.51 \pm 2.60$ & 7.25 \\
\hline $\mathrm{K}_{\mathrm{el}}(1 / \mathrm{h})$ & $0.10 \pm 0.03$ & 0.10 & $0.10 \pm 0.02$ & 0.10 & $0.09 \pm 0.03$ & 0.09 & $0.10 \pm 0.03$ & 0.10 \\
\hline
\end{tabular}

$\mathrm{AUC}_{0-\mathrm{t}}$, area under the drug concentration-time curve from time zero to 48 hours; $A \cup C_{\text {inf, }}$ area under the drug concentration-time curve from time zero to infinity; $C_{\max }$, peak plasma concentration; $K_{e l}$, elimination rate constant; $t_{1 / 2}$, elimination half-life; $T_{\max }$, time to peak concentration. ${ }^{a} T_{\max }$ is given by range and median.

In the six paired comparisons (i.e., between innovator and each generic drug, as well as between the generic drugs), to investigate bioequivalence, In-transformed $C_{\max }, A \cup C_{0-t}$, and $A \cup C_{\text {inf }}$ for the different formulations were similar (see Table 2). The ranges of residual coefficient of variations (CVs) were 17.7-26.5\%, 15.3-24.2\%, and 14.6-23.6\% for $\mathrm{C}_{\max }, \mathrm{AUC}_{0-\mathrm{t}}$, and $\mathrm{AUC}_{\text {inf, }}$ respectively. All six comparisons of the drug formulations met the bioequivalence criteria, with all $90 \% \mathrm{Cls}$ for $\mathrm{C}_{\max }$ and $\mathrm{AUCs}$ being within the 80 $125 \%$ acceptance range. 
Table 2. Summary of ANOVA analysis results of Cmax and AUCS.

\begin{tabular}{|c|c|c|c|}
\hline & $\mathrm{C}_{\max }$ & $A \cup C_{0-t}$ & $A \cup C_{\text {inf }}$ \\
\hline \multicolumn{4}{|c|}{ Generic 1 (B) v.s. Neurontin ${ }^{\circledR}(A)$} \\
\hline Ratio & $100.7 \%$ & $96.9 \%$ & $96.5 \%$ \\
\hline $90 \% \mathrm{Cl}$ & $91.4 \%-110.5 \%$ & $89.5 \%-104.9 \%$ & $89.1 \%-104.4 \%$ \\
\hline Intra-subject CV & $20.2 \%$ & $16.2 \%$ & $15.9 \%$ \\
\hline \multicolumn{4}{|c|}{ Generic 2 (C) v.s. Neurontin ${ }^{\circledR}(A)$} \\
\hline Ratio & $105.1 \%$ & $98.7 \%$ & $98.7 \%$ \\
\hline $90 \% \mathrm{Cl}$ & $93.5 \%-118.3 \%$ & $87.6 \%-111.2 \%$ & $88.0 \%-110.7 \%$ \\
\hline Intra-subject CV & $24.0 \%$ & $24.2 \%$ & $23.6 \%$ \\
\hline \multicolumn{4}{|c|}{ Generic 3 (D) v.s. Neurontin ${ }^{\circledR}(A)$} \\
\hline Ratio & $106.1 \%$ & $98.7 \%$ & $98.3 \%$ \\
\hline $90 \% \mathrm{Cl}$ & $97.1 \%-115.8 \%$ & $91.6 \%-106.4 \%$ & $91.4 \%-105.8 \%$ \\
\hline Intra-subject CV & $17.7 \%$ & $15.3 \%$ & $14.9 \%$ \\
\hline \multicolumn{4}{|c|}{ Generic 1 (B) v.s. Generic 2 (C) } \\
\hline Ratio & $95.8 \%$ & $98.1 \%$ & $97.7 \%$ \\
\hline $90 \% \mathrm{Cl}$ & $84.1 \%-109.0 \%$ & $87.3 \%-110.4 \%$ & $87.2 \%-109.5 \%$ \\
\hline Intra-subject CV & $26.5 \%$ & $24.0 \%$ & $23.3 \%$ \\
\hline \multicolumn{4}{|c|}{ Generic 1 v.s. Generic 3 (D) } \\
\hline Ratio & $94.9 \%$ & $98.1 \%$ & $98.1 \%$ \\
\hline $90 \% \mathrm{Cl}$ & $86.9 \%-103.6 \%$ & $91.0 \%-105.9 \%$ & $91.2 \%-105.4 \%$ \\
\hline Intra-subject CV & $17.7 \%$ & $15.3 \%$ & $14.6 \%$ \\
\hline \multicolumn{4}{|c|}{ Generic 2 (C) v.s. Generic3 (D) } \\
\hline Ratio & $99.1 \%$ & $100 \%$ & $99.6 \%$ \\
\hline $90 \% \mathrm{Cl}$ & $89.9 \%-109.2 \%$ & $91.7 \%-109.1 \%$ & $92.3 \%-109.2 \%$ \\
\hline Intra-subject CV & $19.7 \%$ & $17.7 \%$ & $16.9 \%$ \\
\hline
\end{tabular}

ANOVA, analysis of vanriance; $\mathrm{AUC}_{0-\mathrm{t}}$, area under the drug concentration-time curve from time zero to 48 hours; $\mathrm{AUC}_{\text {inf, }}$ area under the drug concentration-time curve from time zero to infinity; $\mathrm{Cl}$, confidence interval; $C_{\max }$, peak plasma concentration; $C V$, coefficient of variation.

\section{Safety}

No serious adverse events were reported, and there were no apparent differences in the number, type, and severity of adverse events between the different gabapentin formulations. Eighty-three adverse events were reported: 24 events (29\%) in 15 subjects with formulation A, 19 events (23\%) in 15 subjects with formulation B, 20 events (24\%) in 14 subjects with formulation C, and 20 events (24\%) in 12 subjects with formulation $D$. The most commonly reported adverse events were dizziness ( $n=26$ events), somnolence $(n=12)$, and headache $(n=20)$. Overall, 52 events were rated as mild and 32 as moderate; none were rated as severe. Twenty-four adverse events were judged as "probably not related" to the study drug, 23 as "possibly related", and 37 as "probably related". 


\section{DISCUSSION}

To our knowledge, this is the first clinical bioequivalence study to compare multiple generic formulations, in order to investigate issues related to generic-generic interchangeability. We confirmed that the three gabapentin generic formulations currently marketed in the Netherlands are bioequivalent to the innovator (Neurontin ), tested as a single $800 \mathrm{mg}$ dose under fasting conditions. The three generic formulations were also bioequivalent to each other, showing that there is no "drift" with generic-generic exchange. Our findings should add to general trust in generic formulations, and specifically strengthen patients' and physicians' trust in gabapentin generic formulations, so that these formulations can be used instead of branded drugs, the continuing use of which drives up healthcare costs(9).

The gabapentin dose was selected based on our previous simulation study(17) in which we found that the highest dose $(800 \mathrm{mg}$ ) had the broadest range of $90 \% \mathrm{Cls}$ for $\mathrm{C}_{\max }$ and AUC. Therefore, the use of the 800-mg dose could be considered to represent the 'worst case condition' that would most likely result in differences of drug exposure in a clinical setting. However, gabapentin bioavailability decreases with increasing dose $(19,20)$, and so this dose might not be the most relevant dose for finding differences in exposure(13). That said, there appears to be a reasonably linear correlation between dose and bioavailability with single gabapentin doses ranging from $100 \mathrm{mg}$ to $800 \mathrm{mg}$, though with absolute bioavailability ranging from $65 \%$ to $45 \%$ between these doses (20). We consider a single 800-mg dose appropriate to detect potential differences in bioavailability between different formulations, and data can be extrapolated to lower doses.

Our results are consistent with those of our earlier simulation study based on bioequivalence data from the registration files of the Dutch Medicines Evaluation Board(17). The generic:generic ratios for $C_{\max }$ and $A \cup C_{0-t}$ were similar, albeit with narrower $90 \%$ $\mathrm{Cls}$, than those reported in the simulation study. In contrast, Krauss et al. estimated that $17 \%$ of the generic-generic antiepileptic drug comparisons were associated with a greater than 15\% difference between either the lower or upper $90 \% \mathrm{Cl}$ margin (whichever was greater) and 1.0 for $\mathrm{AUC}_{0-\mathrm{t}}$, and concluded that generic-generic switches lead to greater changes in drug exposure than generic-innovator switches (18). However, the $90 \%$ Cls for $\mathrm{AUC}_{0-\mathrm{t}}$ in generic-generic comparisons were estimated based on interstudy simulation and were more likely to show larger differences than generic-innovator comparisons (i.e. intra-study comparison). Moreover, only $2.4 \%$ of the $\mathrm{Cls}_{\text {for }} \mathrm{AUC}_{0-\mathrm{t}}$ in the Krauss study had a lower or upper margin outside the $80-125 \%$ margin, which is in line with our previous simulation results.

As the first clinical bioequivalence study with multiple generic products, our findings help fill the knowledge gap on generic-generic interchangeability. Nevertheless, a few limitations need to be addressed. First, as each formulation was administered only once, within-subject variability could not be assessed. Thus, we cannot predict and 
compare the switchability of branded-generic or generic-generic gabapentin, because, according to Bialer et al., within-subject variability is a key factor in determining the switchability of different formulations $(7,15)$. For instance, if the within-subject variability in drug plasma levels is high, switching to another formulation (innovator or generics), which is usually accompanied by fluctuations in drug plasma levels, may lead to great variations in plasma levels and hence changes in efficacy and safety outcomes. Second, we did not address other factors that may determine effectiveness in clinical practice, such as adherence, which may be particularly relevant in patients with psychiatric disorders $(6,10,21,22)$. It is possible that distrust of generic drugs makes adherence a problem, which means that it may not be possible to predict equivalence in clinical outcomes on the basis of equivalent pharmacokinetics alone when switching from innovator to generic drug. However, the comparable exposure demonstrated in this study, together with the publications from FDA(11) and the regulatory authority in the Netherlands(10) on generic drugs, strongly suggests that there was no clinically relevant difference in exposure to gabapentin among the different formulations. Third, our findings cannot be simply extrapolated to other therapeutic agents because gabapentin is considered to have relatively simple pharmacokinetic properties, i.e. high solubility, active transport through cells, and renal excretion. For drugs with complex pharmacokinetic properties, pharmacokinetic data would be more variable than was the case for the gabapentin formulations, and $90 \% \mathrm{Cls}$ for $\mathrm{AUC}$ and $\mathrm{C}_{\max }$ would be broader within the accepted $80-125 \%$ limits. Bioequivalence studies with drugs with more complex pharmacokinetics are needed to exclude "drift" with generic-generic substitution; however, if the innovator: generic ratios for AUC are close to 1.0, then it is likely that the comparison between generic formulations would be within the acceptable limits $(11,17)$.

The adverse events most frequently reported by the subjects were in line with those reported in the Summary of Product Characteristics (SPC) for Neurontin (19). No relevant differences in safety profiles were observed between the different gabapentin formulations, particularly with respect to the number and pattern of adverse events, consistent with other reports of drug switches $(3,15,23,24)$. A recent case-control study based on a health-care database(6) showed that refilling the prescription with the same product, regardless of whether that product was an innovator or generic drug, was associated with an approximately 2-fold increase in the odds of emergency treatment for seizure. However, the refill-adjusted effect of generic-innovator or genericgeneric switches was small, with only a $4-19 \%$ increase in the odds of seizure-related outcomes. According to the authors of this paper, this indicated that the switches between formulations may not be primarily responsible for the increased occurrence of adverse events. Instead, the increased breakthrough seizures may be the consequence of a lapse in pharmacotherapy continuity prior to the prescription refilling. Or, it may be related to the recurrence of subtle neurological symptoms that prompted the refill. Moreover, a recent review reported a low risk of a poor clinical outcome after switching 
to a generic formulation(23). Furthermore, a systematic review and meta-analysis by Kesselheim et al. did not detect differences in uncontrolled seizures between generic medications and branded medications (the aggregate odds ratio=1.0; 95\% $\mathrm{Cl}$ : 0.7-1.4) (24).

The complexity of the issues surrounding the use of generic drugs warrants wellcontrolled epidemiological studies, clinical trials, and simulation studies involving subpopulations or subgroups of drugs $(5,7,18,25)$. Close monitoring of patients after generic substitution has been recommended $(10,23)$. Several authors have emphasized the importance of within-subject variability, particularly in antiepileptic treatments, and recommended a replicated study design for addressing the issue of switchability (15, 22). Given the maximum intra-subject $C V$ s of $26.5 \%$ for $C_{\max }$ and $24.2 \%$ for $A \cup C_{0-t}$ (Table 2 ), issues with generic interchangeability may still exist on an individual basis.. Further research into this individual bioavailability is warranted.

In conclusion, we established the bioequivalence of four gabapentin formulations (Neurontin ${ }^{\circ}$ and three registered generic formulations of gabapentin) in healthy subjects. The results indicate the absence of "drift" with gabapentin generic-generic exchange. Moreover, our study supports the conclusions of a previous study, namely, that gabapentin generics are likely to be interchangeable, because the point estimate is very close to 1.0. Since this is also the case for other approved generic drugs, there would appear to be a low likelihood of drift in generic-generic exchange with other medicinal products as well.

\section{METHODS}

\section{Treatments}

Four medicinal products containing $800 \mathrm{mg}$ gabapentin registered in the Netherlands (i.e., the innovator "Neurontin " and three generic gabapentin formulations) were used (Table 3). All medicinal products were supplied by the pharmacy of the Maastricht University Medical Centre (MUMC+).

Table 3. Characteristics of gabapentin formulations used in the study.

\begin{tabular}{|c|c|c|c|c|}
\hline & $\begin{array}{l}\text { Innovator } \\
\text { (Treatment A) }\end{array}$ & $\begin{array}{l}\text { Generic } 1 \\
\text { (Treatment B) }\end{array}$ & $\begin{array}{l}\text { Generic } 2 \\
\text { (Treatment C) }\end{array}$ & $\begin{array}{l}\text { Generic } 3 \\
\text { (Treatment D) }\end{array}$ \\
\hline Name & Neurontin 800 & $\begin{array}{l}\text { Gabapentine } \\
\text { Apotex } 800 \text { mg }\end{array}$ & $\begin{array}{l}\text { Gabapentine } \\
800 \mathrm{PCH}\end{array}$ & $\begin{array}{l}\text { Gabapentine } \\
\text { CF } 800 \text { mg }\end{array}$ \\
\hline Unit dose & $800 \mathrm{mg}$ & $800 \mathrm{mg}$ & $800 \mathrm{mg}$ & $800 \mathrm{mg}$ \\
\hline Expiry date & $08 / 2012$ & $06 / 2012$ & $01 / 2013$ & $04 / 2014$ \\
\hline $\begin{array}{l}\text { Dutch registration } \\
\text { number (RVG) and } \\
\text { registration date }\end{array}$ & $\begin{array}{l}25248 ; \\
27 / 11 / 2000\end{array}$ & $\begin{array}{l}\text { 34333; } \\
\text { 24/01/2008 }\end{array}$ & $\begin{array}{l}\text { 31984; } \\
\text { 11/04/2005 }\end{array}$ & $\begin{array}{l}\text { 101081; } \\
\text { 20/01/2009 }\end{array}$ \\
\hline
\end{tabular}




\section{Study Design}

The study was designed as a single-dose, randomized, four-treatment, four-period, crossover bioequivalence study in healthy subjects under fasting conditions. There was a washout period of at least 7 days between the administration of different formulations. Each subject randomly received a single dose of $800 \mathrm{mg}$ of gabapentin (either the branded Neurontin tablet or one of the three generic gabapentin tablets) after an overnight fast, according to a randomization schedule for a four-way crossover design $\operatorname{study}(26)$.

\section{Study Population}

The study population consisted of non-smoking or moderately smoking (i.e. fewer than 10 cigarettes a day, for at least 3 months) healthy volunteers. The sample size was calculated based on conditions observed from actual bioequivalence studies (submitted to our regulatory authority for generic gabapentin registration). Available data from four registration studies of generics gabapentin $(800 \mathrm{mg}$ ) showed intra-subject CV varying from 0.16 to 0.26 for $C_{\max }$ and AUC. Therefore, we decided to assume a intra-subject CV of 0.25 . Furthermore, actual bioequivalence studies indicated that assuming 1.04 as the (test/reference) ratio of gabapentin in the two formulations was reasonable. According to these assumptions, the sample size was calculated based on a $2 \times 2$ cross-over design with a predefined power of 75\%, type I error (alpha) of 0.05 and equivalence limits of $80-125 \%$, as we were primarily interested in pairwise comparisons of each generic formulation versus the reference. The $80-125 \%$ limits are routinely used in bioequivalence studies $(12,13)$, and obeying these margins is assumed to indicate a lack of relevance of any differences in exposure. Using the method as described by Diletti et al.(27) and Zhang (28), we found that at least 23 subjects were needed to have the $75 \%$ power, and by considering possible dropout, a sample size of 24 subjects was chosen. Main inclusion criteria were age, body weight, BMI, and general health status (medical history, physical examination, vital signs, routine laboratory tests and 12-lead ECG), and exclusion criteria were history of diseases and concomitant medication use, viral infections, hypersensitivity to gabapentin, nutrition pattern, and pregnancy.

\section{Measurements}

Blood samples were taken at baseline and at $t=0.5,1.0,1.5,2.0,2.5,3.0,3.5,4.0,4.5$, $5.0,6.0,8.0,12,24,36$, and 48 hours after dosing. Samples were collected in ethylenediaminetetraacetic acid (EDTA)-coated tubes, cooled in an ice bath, and centrifuged (4000 rpm) at $18{ }^{\circ} \mathrm{C}$ for 5 minutes. Plasma samples were divided into two aliquots and stored at $-20{ }^{\circ} \mathrm{C}$ pending shipment for assay. The plasma concentrations of gabapentin were measured using a validated ultra-performance liquid chromatography-mass spec- 
trometry (UPLC-MS)(29). Lower limit of quantification was $0.03 \mathrm{mg} / \mathrm{L}$ and the calibration curve ranged from $0.03-25 \mathrm{mg} / \mathrm{L}(30)$. The within- and between-run accuracy and precision were within $15 \%$ of the expected value at the low-, medium-, and high-quality control levels. Gabapentin in plasma samples was stable during storage.

\section{Pharmacokinetic Analyses}

Plasma gabapentin concentrations were used to calculate pharmacokinetic parameters $\left(A \cup C_{0-t}, A \cup C_{\text {inf }}, C_{m a x}\right.$, time to peak concentration, elimination half-life, residual area and elimination rate constant) for each formulation and subject, using WinNonLin (Version 5.3; Pharsight, Cary, NC).

\section{Statistical Analyses}

Analysis of variance (ANOVA) was performed on In-transformed $A \cup C_{0-t}, A \cup C_{\text {inf }}$, and $C_{\text {max }}$. The ANOVA model included sequence, treatment, period, and subject within sequence as fixed effects, and subject as a random effect. The least-squares mean (LSM), the difference between formulation LSM, and the standard error associated with this difference were calculated.

Ratios of LSM between formulations were calculated by exponentiation of the LSM derived from the analyses of the In-transformed $A_{U C} C_{0-t}, A \cup C_{\text {inf }}$, and $C_{\max }$. The $90 \% \mathrm{Cls}$ for the ratios were derived by exponentiation of the $\mathrm{Cl}$ s obtained for the difference between formulation LSM in the same analyses. Bioequivalence with gabapentin was considered proven if the $90 \% \mathrm{Cls}$ of the ratio of LSM from the ANOVA of the In-transformed $\mathrm{C}_{\text {max }}, \mathrm{AUC}_{0-\mathrm{t}}$, and $A \cup \mathrm{C}_{\text {inf }}$ were within $80-125 \%$ limit, in accordance with the EMA Guideline on the Investigation of Bioequivalence (January 2010, London)(13). All statistical analyses were conducted using SPSS 18.0 (SPSS, Chicago, IL).

\section{Safety Analyses}

Safety was monitored on the basis of routine laboratory tests, vital signs, 12-lead ECG, and adverse events. An investigator recorded the number, type, severity, and relationship of adverse events to drug administration.

\section{Ethics}

This clinical study (NTR2964) and informed consent forms were approved by the Independent Ethics Committee in the Netherlands prior to subject screening and enrolment. This study was conducted according to the principles of the Declaration of Helsinki (version 2007-2008) and in accordance with requirements of the Medical Research Involving Human Subjects Act (WMO) and Good Clinical Practice (GCP) regulations. 


\section{ACKNOWLEDGMENTS}

The study described was supported by Medicine Evaluation Board (CBG-MEB) in the Netherlands. Its contents are solely the responsibility of the authors and do not necessarily represent the official view of CBG-MEB.

The authors would like to acknowledge Dr. Christine Gispen-de Wied, Mrs. Angela Colbers and Dr. Ingrid Scheffers, and the Department of Clinical Pharmacology and Pharmacy and The Epilepsy Institute in the Netherlands (SEIN) of Amsterdam free University Medical Centre for their expert assistance during the conduct of this study. 


\section{REFERENCES}

(1) Meredith, P.A. Potential concerns about generic substitution: bioequivalence versus therapeutic equivalence of different amlodipine salt forms. Curr Med Res Opin 25, 2179-89 (2009).

(2) Crawford, P., Hall, W.W., Chappell, B., Collings, J. \& Stewart, A. Generic prescribing for epilepsy. Is it safe? Seizure 5, 1-5 (1996).

(3) Wilner, A.N. Therapeutic equivalency of generic antiepileptic drugs: results of a survey. Epilepsy Behav 5 , 995-8 (2004).

(4) Borgheini, G. The bioequivalence and therapeutic efficacy of generic versus brand-name psychoactive drugs. Clin Ther 25, 1578-92 (2003).

(5) Privitera, M.D. Generic antiepileptic drugs: current controversies and future directions. Epilepsy Curr 8, 113-7 (2008).

(6) Gagne, J.J., Avorn, J., Shrank, W.H. \& Schneeweiss, S. Refilling and switching of antiepileptic drugs and seizure-related events. Clin Pharmacol Ther 88, 347-53 (2010).

(7) Bialer, M. Generic products of antiepileptic drugs (AEDs): is it an issue? Epilepsia 48, 1825-32 (2007).

(8) Sankar, R. \& Glauser, T.A. Understanding therapeutic equivalence in epilepsy. CNS Spectr 15, 112-23 (2010).

(9) Duerden, M.G. \& Hughes, D.A. Generic and therapeutic substitutions in the UK: are they a good thing? Br J Clin Pharmacol 70, 335-41 (2010).

(10) Maliepaard, M. et al. Requirements for generic anti-epileptic medicines: a regulatory perspective. J Neurol 256, 1966-71 (2009).

(11) Davit, B.M. et al. Comparing generic and innovator drugs: a review of 12 years of bioequivalence data from the United States Food and Drug Administration. Ann Pharmacother 43, 1583-97 (2009).

(12) Guidance for Industry. Bioavailability and Bioequivalence. Studies for Orally Adminsitered Drug ProductsGeneral Considerations (US Food and Drug Administration Center for Drug Evaluation and Research, Washington, 2003).

(13) Guidline on the Investigation of Bioequivalence. (Committee for Medicinal Products for Human use (CHMP), European Medicine Agency,, London, 2010).

(14) Moore, N., Berdai, D. \& Begaud, B. Are generic drugs really inferior medicines? Clin Pharmacol Ther 88 , 302-4 (2010).

(15) Bialer, M. \& Midha, K.K. Generic products of antiepileptic drugs: a perspective on bioequivalence and interchangeability. Epilepsia 51, 941-50 (2010).

(16) Anderson, S. \& Hauck, W.W. The transitivity of bioequivalence testing: potential for drift. Int J Clin Pharmacol Ther 34, 369-74 (1996).

(17) Maliepaard, M., Banishki, N., Gispen-de Wied, C.C., Teerenstra, S. \& Elferink, A.J. Interchangeability of generic anti-epileptic drugs: a quantitative analysis of topiramate and gabapentin. Eur J Clin Pharmacol 67, 1007-16 (2011).

(18) Krauss, G.L., Caffo, B., Chang, Y.T., Hendrix, C.W. \& Chuang, K. Assessing bioequivalence of generic antiepilepsy drugs. Ann Neurol 70, 221-8 (2011).

(19) Summary of Product Characteristics: Neurontin Capsules and Tablets. <http://www.medicines.org. uk/emc/medicine/17095>. Accessed 01/08/2012.

(20) Gidal, B.E. et al. Gabapentin bioavailability: effect of dose and frequency of administration in adult patients with epilepsy. Epilepsy Res 31, 91-9 (1998).

(21) Peterson, G.M. Generic Substitution of Antiepileptics: Need for a Balanced View. CNS Spectr, (2011); epub ahead of print 1 August 2011.

(22) Peterson, G.M. Generic substitution: a need for clarification. Br J Clin Pharmacol 71, 966-7; author reply 8 (2011).

(23) Bobo, W.V., Stovall, J.A., Knostman, M., Koestner, J. \& Shelton, R.C. Converting from brand-name to generic clozapine: a review of effectiveness and tolerability data. Am J Health Syst Pharm 67, 27-37 (2010). 
(24) Kesselheim, A.S. et al. Seizure outcomes following the use of generic versus brand-name antiepileptic drugs: a systematic review and meta-analysis. Drugs 70, 605-21 (2010).

(25) Meredith, P. Bioequivalence and other unresolved issues in generic drug substitution. Clin Ther 25, 287590 (2003).

(26) Jackson, A.J. (ed.). Generics and Bioequivalence (CRC Press, 1994).

(27) Diletti, E., Hauschke, D. \& Steinijans, V.W. Sample size determination for bioequivalence assessment by means of confidence intervals. International journal of clinical pharmacology, therapy, and toxicology 29, 1-8 (1991).

(28) Zhang, P. A simple formula for sample size calculation in equivalence studies. Journal of biopharmaceutical statistics 13, 529-38 (2003).

(29) Ramakrishna, N.V. et al. Rapid quantification of gabapentin in human plasma by liquid chromatography/tandem mass spectrometry. J Pharm Biomed Anal 40, 360-8 (2006).

(30) Chahbouni, A. et al. Rapid quantification of gabapentin, pregabalin, and vigabatrin in human serum by ultraperformance liquid chromatography with mass-spectrometric detection. Ther Drug Monit 35, 48-53 (2013). 



\section{Chapter}

\section{Investigation into the interchangeability of generic formulations using immunosuppressants and a broad selection of medicines}

$\underline{\text { Yang } \mathrm{Yu}^{1,2}}$, Steven Teerenstra ${ }^{2,3}$, Cees Neef ${ }^{1,4}$, David Burger ${ }^{5}$ and Marc Maliepaard ${ }^{2}$

${ }^{1}$ Dept. Pharmacology and Toxicology, CARIM, Maastricht University Medical Centre, Maastricht, The Netherlands

${ }^{2}$ Medicines Evaluation Board, Utrecht, The Netherlands

${ }^{3}$ Dept. for Health Evidence, section Biostatistics, Radboud university medical centre, Nijmegen, The Netherlands

${ }^{4}$ Dept. Pharmacology and Toxicology, CAPHRI, Maastricht University Medical Centre, Maastricht, The Netherlands

${ }^{5}$ Dept. of Pharmacy, Radboud University Nijmegen Medical Centre, Nijmegen, The Netherlands

European Journal of Clinical Pharmacology 71, 979-990 (2015) 


\section{ABSTRACT}

Purpose To date, the interchangeability of generic drugs has only been investigated for a limited number of medicines. The objective of this study was to investigate genericgeneric drug interchangeability in a large subset of generic formulations in order to cover a broad spectrum of drugs.

Methods Orally administered drugs for investigation in this study were selected using strict, pre-defined criteria, with the purpose to avoid bias. This selection procedure yielded atorvastatin, bicalutamide, naratriptan, olanzapine, perindopril and venlafaxine. Further, ciclosporin, tacrolimus and mycophenolate mofetil were investigated as test immunosuppressants. Adjusted indirect comparisons were conducted between generic drugs containing the same active substance and the $90 \%$ confidence interval $(\mathrm{Cl})$ for AUC and $\mathrm{C}_{\max }$ were calculated.

Results In total, 120 bioequivalence studies were identified in the Dutch medicine regulatory agency's database, allowing 292 indirect comparisons between generic drugs. The indirect comparison results indicated that in the vast majority of cases, i.e., $80.5 \%$, the $90 \% \mathrm{Cls}$ for both $\mathrm{AUC}_{\mathrm{t}}$ and $\mathrm{C}_{\max }$ fell within the bioequivalence criteria (in $90.1 \%$ and $87.0 \%$ for $A \cup C_{t}$ and $C_{\max }$, respectively). In $1 \%$ of the 292 indirect comparison for $A \cup C_{t}$ and $3 \%$ for $C_{\max }$, a wider range of $75-133 \%$ (or $80-125 \%$ ) was exceeded.

Conclusions Overall, our study suggests that exposure-related risks associated with the exchange of different generic drugs in clinical practice is not increased to a relevant extent compared to the situation in which a generic is exchanged with the innovator.

Key words: Generic drugs, interchangeability, bioequivalence, pharmacokinetics 


\section{INTRODUCTION}

A generic medicinal product is considered to be therapeutically equivalent to the innovator. To be registered, one or more so-called bioequivalence studies are required to demonstrate that the $90 \%$ confidence interval $(\mathrm{Cl})$ for the generic : innovator ratios of the area under the drug concentration-time curve (AUC) and the maximum concentration $\left(\mathrm{C}_{\max }\right)$ are within the range of $80-125 \%(1-4)$. This acceptance range of $80-125 \%$ can be widened based on a scaled approach for $C_{\max }$ up to $69.84-143.19 \%$ for highly variable drugs or can be tightened to $90-111.11 \%$ for narrow therapeutic index drugs (NTIDs).

At present, for most active pharmaceutical ingredients (APIs), multiple generic products have been approved in the Netherlands, and regular switches from one generic product to another are seen in clinical practice. From a regulator's perspective, generic-generic drug switching is unlikely to impact treatment, as all generic formulations have been shown to be bioequivalent to the innovator product, and thus the deviation of drug exposure between generic products should be limited (5). Based on that, generic products are considered to be sufficiently comparable to each other. However, current regulation does not require bioequivalence studies between different generic formulations. In fact, in theory, a "drift" may appear upon generic-generic drug substitution, meaning that generic formulations that are bioequivalent to the innovator drug, respectively, may not be bioequivalent to each other (6). This potential problem is due to the acceptance range for generic product registration $\left(90 \% \mathrm{Cls}\right.$ of $\mathrm{AUC}$ and $\mathrm{C}_{\max }$ ratios within the $80-125 \%$ range), which allows small variations in exposure between generic and innovator drugs. Thus, if one generic product has a higher and another has a lower exposure level than the innovator drug, the difference between the generic drugs will be reinforced, potentially leading to bio-inequivalence between them. In addition, the possibility of the occurrence of bio-inequivalence between generic drugs is also shown in theoretical Monte-Carlo simulation studies $(7,8)$. Therefore, investigation into this generic-generic drug comparability is warranted.

In light of the discussion about the drift upon generic drug substitutions, we have previously conducted indirect comparisons to evaluate generic-generic drug interchangeability using gabapentin and topiramate as test medications (9). As a result, in general, bioequivalence between the different generic gabapentin and topiramate formulations was demonstrated. These interstudy comparison results were subsequently confirmed and validated by a clinical bioequivalence study using multiple generic formulations of gabapentin (10). Furthermore, in the public domain, the comparability and safety of generic immunosuppressants, typically in transplantation medicines, are hot topics of discussion (11-17). Therefore, this study is conducted to investigate the acceptability of generic-generic drug interchangeability for a broad spectrum of medicines as well as immunosuppressants based on the bioequivalence studies submitted for registration. 


\section{METHODS}

\section{Drug selection}

For this study, only orally administrated tablets or capsules with systemic action were considered relevant. The database at Dutch medicine regulatory agency was used for drug selection and data collection. To avoid a selection bias, a period of January 1 to May 9, 2008 was pre-defined to create a cohort, which contains all APIs that had at least one generic formulation (tablets or capsules) registered during this period, regardless of the type of registration procedure. Second, the identified APIs were ordered according to the registration date starting from January 1, 2008. In order to largely predict the interchangeability of currently marketed generic drugs, it was pre-defined to select the first six APIs from the initial cohort that had more generic drugs registered after January 1, 2008 (until June 8, 2010). In addition to this pre-defined selection, we also decided to investigate cyclosporine, tacrolimus and mycophenolate mofetil as test immunosuppressants, triggered by the debate in the field on generic drugs from this class. For all selected APIs, bioequivalence studies in the registration files of generic formulations before January 1, 2012 were retrieved from the Dutch medicine regulatory agency's database.

\section{Data analysis}

Adjusted indirect comparisons between generic products containing the same API were conducted. This method has been well-used in this kind of research, since it allows an estimation of the $90 \% \mathrm{Cl}$ based on an inter-study comparison. The major limitation is that the uncertainty (i.e. standard error) of the indirect comparison is larger than the standard error of any of the studies under comparison, which is expected to be also larger than the standard error in case two generic drugs are directly compared in a study. It therefore leads to an over-estimation of the differences between generic drugs (i.e. broader $90 \% \mathrm{Cls}$ than calculated in a direct comparison). Thus, the adjusted indirect comparison method is considered as a conservative approach and is expected to provide reliable results in the case that the adjusted indirect comparison indicates the $90 \%$ Cls within the acceptance range of $80-125 \%$, as verified by an in vivo bioequivalence study (10). In addition, analogous to the method used in the direct comparison, the chosen indirect comparison method is an average bioequivalence approach, which allows to compare the results of the indirect comparisons with the results of in vivo bioequivalence studies, because this is the criterion used for the studies for generic drug registration. Thus, this method is preferred over other methods recommended in literature based on population and individual bioequivalence approach $(18,19)$ and scaled-average bioequivalence approach (20). 
The indirect comparisons were only made between two generic formulations when their bioequivalence studies used the same dose, design and conditions (i.e. either fasting/fed and/or either crossover/parallel). In line with recommendations by Gwaza et al.(21), a pragmatic method based on $t$ test was used (see the algorithm below). This method has been shown to give comparable results as the homoscedastic method. All analyses were performed using Excel, Microsoft Office $2010^{\circ}$.

\section{Algorithms}

All calculations were based on In-transformed data. The ratios between two generic formulations (G2/G1) for $A \cup C$ ( $A \cup C_{0-t}$ for single dose studies and $A \cup C_{T}$ for one dosing interval at steady state for multiple-dose studies) and $C_{\max }$ were calculated by subtraction of the In-transformed generic:innovator ratios in one bioequivalence study $\left(R_{B E 1}\right)$ from the ratios in another bioequivalence study $\left(R_{B E 2}\right)$, which gives the adjusted difference between $\mathrm{G} 1$ and $\mathrm{G} 2$ :

Equation 1:

$$
\ln R_{(G 2 / G 1)}=\ln R_{B E 2}-\ln R_{B E 1}
$$

The $90 \% \mathrm{Cls}$ were calculated as:

Equation 2:

Equation 3:

$$
\begin{aligned}
& S E_{(d)}=\sqrt[2]{S E_{1}^{2}+S E_{2}^{2}} ; \quad\left(\text { d.f. }=\mathrm{n}_{1}+\mathrm{n}_{2}-2\right) \\
& \text { In-transformed } 90 \% \mathrm{CI}=\ln R_{(G 2 / G 1)} \pm t(d f) \times S E_{(d)}
\end{aligned}
$$

The standard errors from the ANOVA-model in the bioequivalence studies $\left(\mathrm{SE}_{1}\right.$ and $\mathrm{SE}_{2}$ ) were used to estimate the standard error $\left(\mathrm{SE}_{(\mathrm{d})}\right)$ in the indirect comparison of $\mathrm{G} 2$ and Degrees of freedom (d.f.) were estimated as the sum of subjects from the two bioequivalence studies $\left(n_{1}\right.$ and $\left.n_{2}\right)$ minus two, and student's $t$-distribution t-percentiles were used. The exponentiated results from Equation 1 and 3 were used to judge bioequivalence between generics.

\section{Definition of bioequivalence}

The European Medicines Agency (EMA) guidelines $(2,22)$ regarding bioequivalence were followed, i.e., $90 \% \mathrm{Cls}$ for both $\mathrm{AUC}$ and $\mathrm{C}_{\max }$ of a generic drug with immediate release properties should meet the $80-125 \%$ criterion. Further guidance on NTIDs were adhered for cyclosporine, tacrolimus and mycophenolate mofetil (23). The tightened acceptance range of $90-111 \%$ was applied for both $A \cup C$ and $C_{\max }$ for cyclosporine, and only AUC for tacrolimus. Bioequivalence of generic mycophenolate mofetil was demonstrated based on the plasma concentration of mycophenolic acid and the $80-125 \%$ criterion was followed. 


\section{RESULTS}

Following the selection criteria, atorvastatin, bicalutamide, naratriptan, olanzapine, perindopril and venlafaxine were included for this study. Further, the immunosuppressants cyclosporine, tacrolimus and mycophenolate mofetil were included. Overall, eight of the selected APIs were immediate release formulations, whereas one, i.e. venlafaxine, was an extended release formulation.

\section{Clinical Bioequivalence Studies}

For the selected 9 APIs, 115 brands of generic drugs were identified, which were registered based on 120 bioequivalence studies in total (Table 1). A number of different brands of generic products were registered based on the same dossier, i.e. the same bioequivalence study(ies). The generic : innovator ratios for AUC and $\mathrm{C}_{\max }$ in the 120 studies ranged from $90.0 \%$ to $116.7 \%$ and from $87.7 \%$ to $118.5 \%$, respectively (Supplementary I). The mean absolute deviation of the ratios from $100 \%$ in this set of generics was $4.5 \%$ for $A U C$ and $5.1 \%$ for $C_{\max }$, respectively. The ranges of the lower and upper boundary of the $90 \% \mathrm{Cls}$ for $\mathrm{AUC}$ and $\mathrm{C}_{\max }$ over the available bioequivalence studies are summarized for every API and strength in Table 1 (below).

\section{Adjusted indirect comparisons between generic drugs}

In total, 292 indirect comparisons between generic drugs were conducted based on 116 bioequivalence studies. Four bioequivalence studies could not be used as they were the only bioequivalence study for a specific strength of APIs (Table 2). In 80.5\% (235 out of 292) of the comparisons, the $90 \% \mathrm{Cls}$ for both $\mathrm{AUC}$ and $\mathrm{C}_{\max }$ fell within the $80-125 \%$ (or 90-111\%) acceptance range. In 90.1\% (263 out of 292) and in $87.0 \%$ (254 out of 292) of the comparisons, the estimated $90 \% \mathrm{Cls}$ were within the predefined acceptance range for $A \cup C$ and $C_{\max }$, respectively (Table 2 ). 


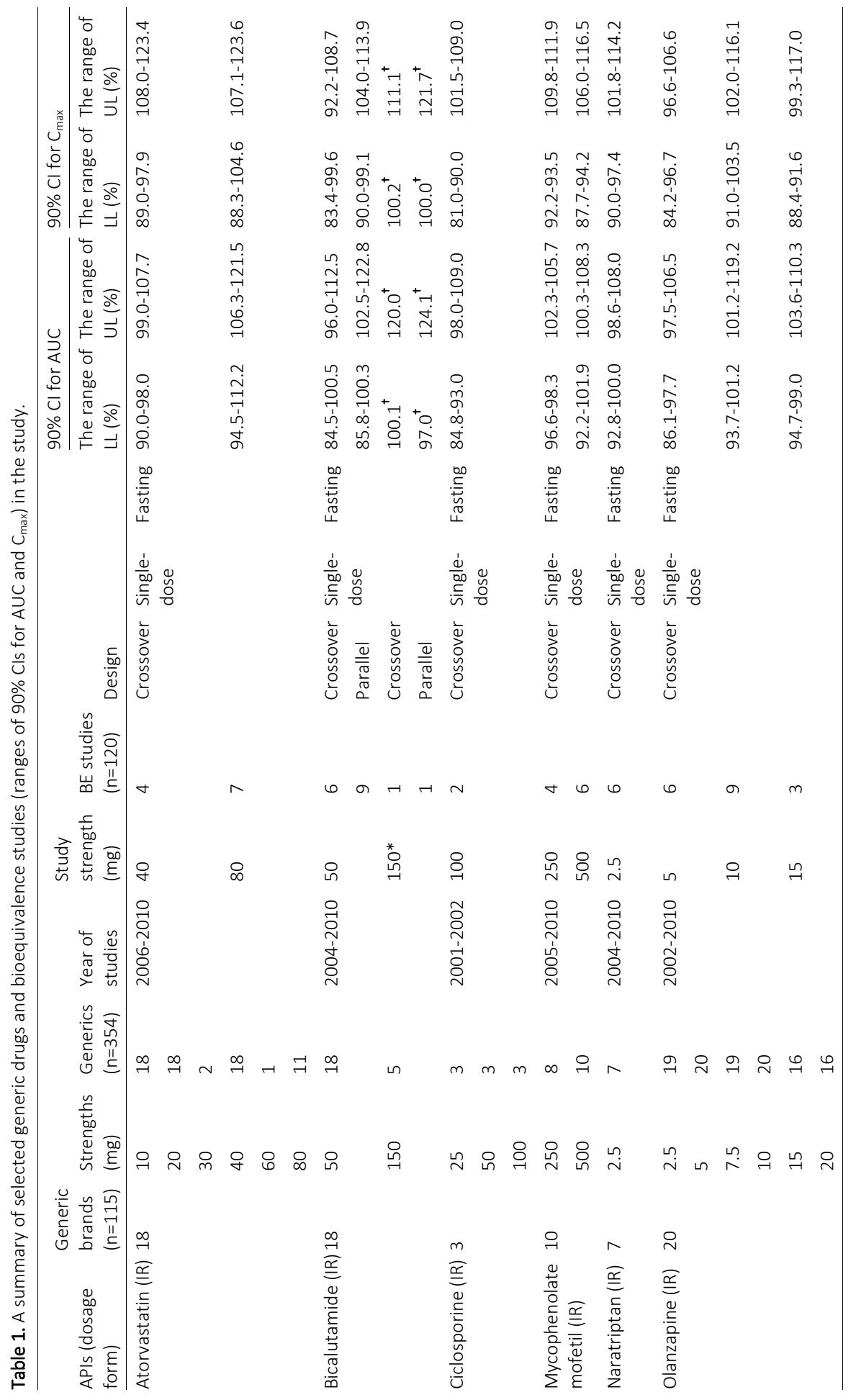




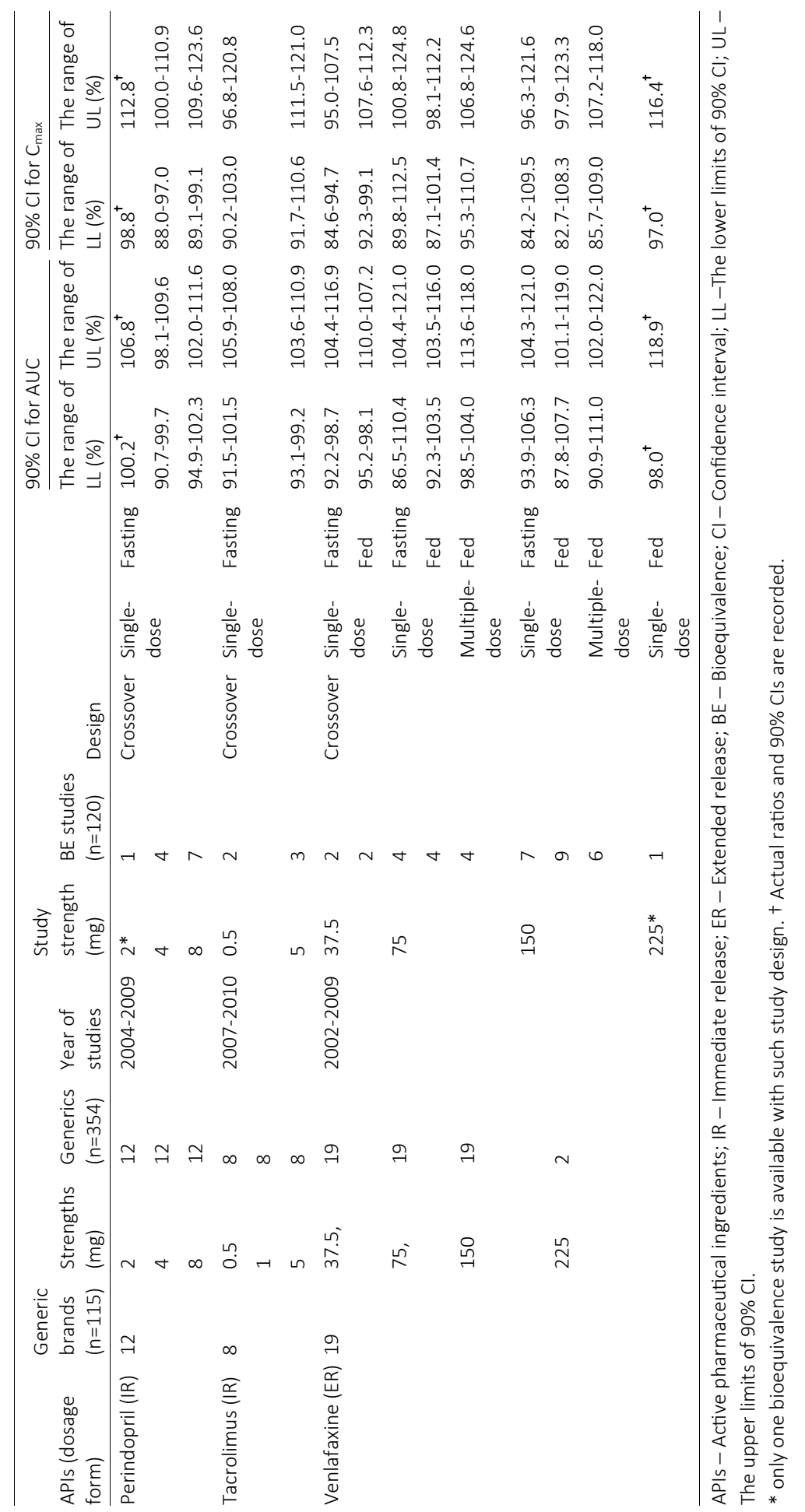




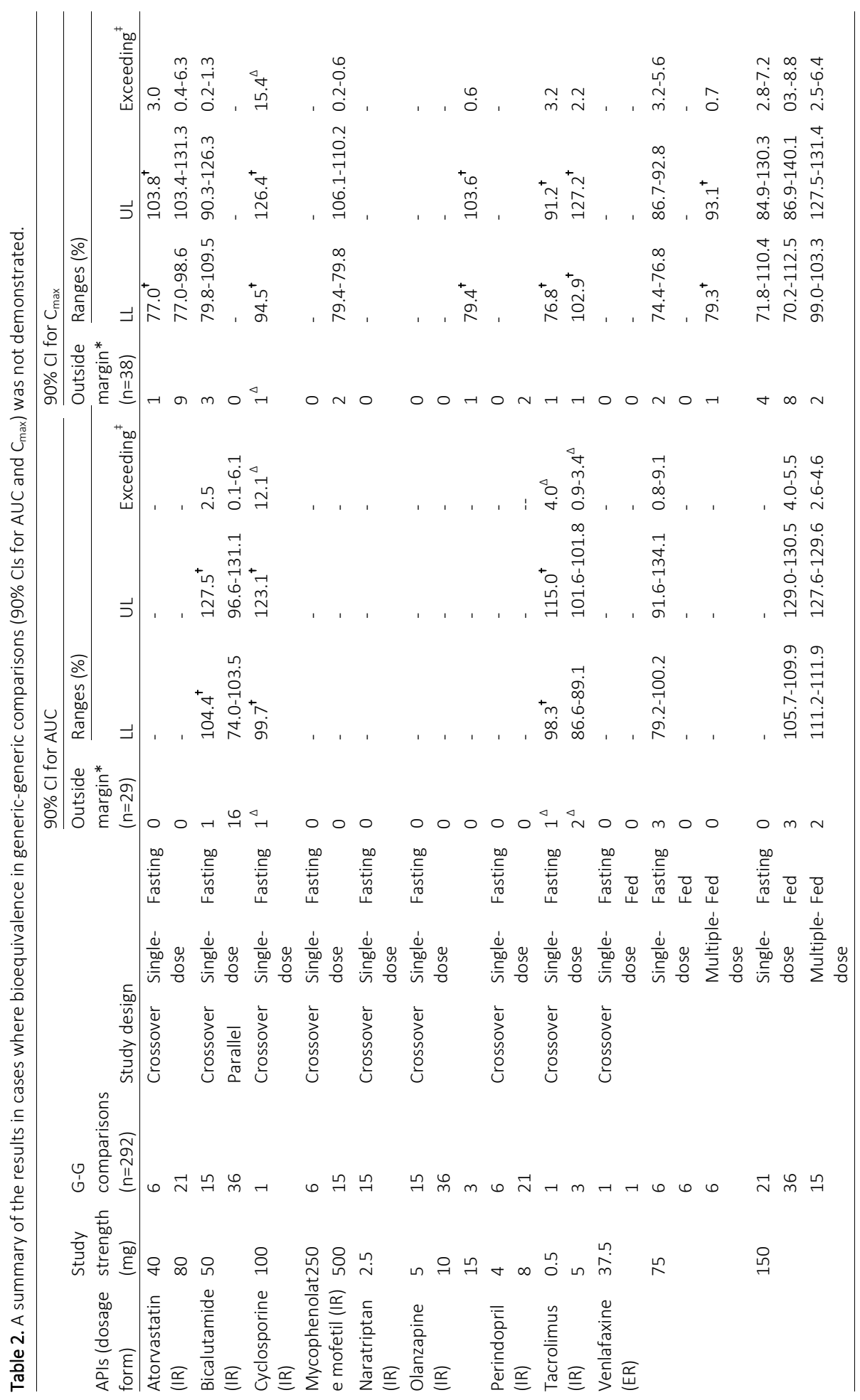


APIs - Active pharmaceutical ingredients; IR - Immediate release; ER - Extended release; BE - Bioequivalence; G-G - Generic and generic; $\mathrm{Cl}$ - Confidence interval; $\mathrm{LL}-$ The lower limits of $90 \% \mathrm{Cl}$; UL - The upper limits of $90 \% \mathrm{Cl}$.

* The margin is $80-125 \%,{ }^{\triangle}$ for some cases $90-111 \%$ margin is applied. ${ }^{\dagger}$ Actual ratios and $90 \% \mathrm{Cls}$ are recorded for single G-G comparisons. ¥The percentages of the maximum exceeding of the boundary are recorded, compared to the margin of $80-125 \%$ (or $90-111 \%$ ).

\section{Area under the plasma concentration-time curve (AUC)}

The estimated generic : generic ratios for AUC ranged from $84.2 \%$ to $120.4 \%$ (Fig. 1a). The mean absolute deviation of the ratios from $100 \%$ in this set of generic drugs was $5.4 \%$. The individual lower and upper boundaries of $90 \% \mathrm{Cls}$ for AUC varied in the ranges of $72.9-111.9 \%$ and $91.6-134.1 \%$, respectively (Fig. 2a).

All generic-generic $90 \% \mathrm{Cls}$ for AUC were within the $80-125 \%$ acceptance criterion for atorvastatin, mycophenolate mofetil, naratriptan, olanzapine and perindopril. For relatively few comparisons (29 out of 292, see Figure 2a and Table 2), 90\% Cls for generic-generic AUC ratios did not meet the $80-125 \%$ (or $90-111 \%$ ) criterion. The level by which the margin of $80 \%$ or $125 \%$ (or 90 or $111 \%$ ) was exceeded ranged from $0.1 \%$ to $12.1 \%$. Twenty-six of these 29 cases did not exceed a wider range of $75-133 \%$ (or 80 $125 \%)$. Thus, overall, in $1 \%$ of the 292 indirect comparisons for $A \cup C_{t}$ a wider range of $75-133 \%$ (or $80-125 \%$ ) was exceeded.

\section{Maximum plasma concentration $\left(C_{\max }\right)$}

The generic : generic ratios for $C_{\max }$ ranged from $78.1 \%$ to $124.5 \%$ (Fig. $1 \mathrm{~b}$ ) based on the indirect comparisons. The mean absolute deviation of the ratios from $100 \%$ in this set of generic drugs was $6.1 \%$. The individual lower and upper boundaries of $90 \% \mathrm{Cls}$ for $\mathrm{C}_{\max }$ ranged from $70.2 \%$ to $112.5 \%$ and from $84.9 \%$ to $140.1 \%$, respectively. Similar with the situation for $A \cup C$, in the majority of generic-generic drug comparisons, a $90 \% \mathrm{Cl}$ for $\mathrm{C}_{\max }$ within the acceptance ranges was obtained (Fig. 2b and Table 2). A 90\% Cl exceeding the $80-125 \%$ margin (or 90-111\%) was observed for 38 of the 292 comparisons (Table 2 ). The level by which the margin of $80 \%$ or $125 \%$ (or 90 or $111 \%$ ) was exceeded ranged from $0.1 \%$ to $15.4 \%$. Twenty-nine of these 38 cases did not exceed a wider range of 75 $133 \%$ (or $80-125 \%$ ). Thus, overall, in $3 \%$ of the 292 indirect comparisons for $C_{\max }$ a wider range of $75-133 \%$ (or $80-125 \%$ ) was exceeded. 


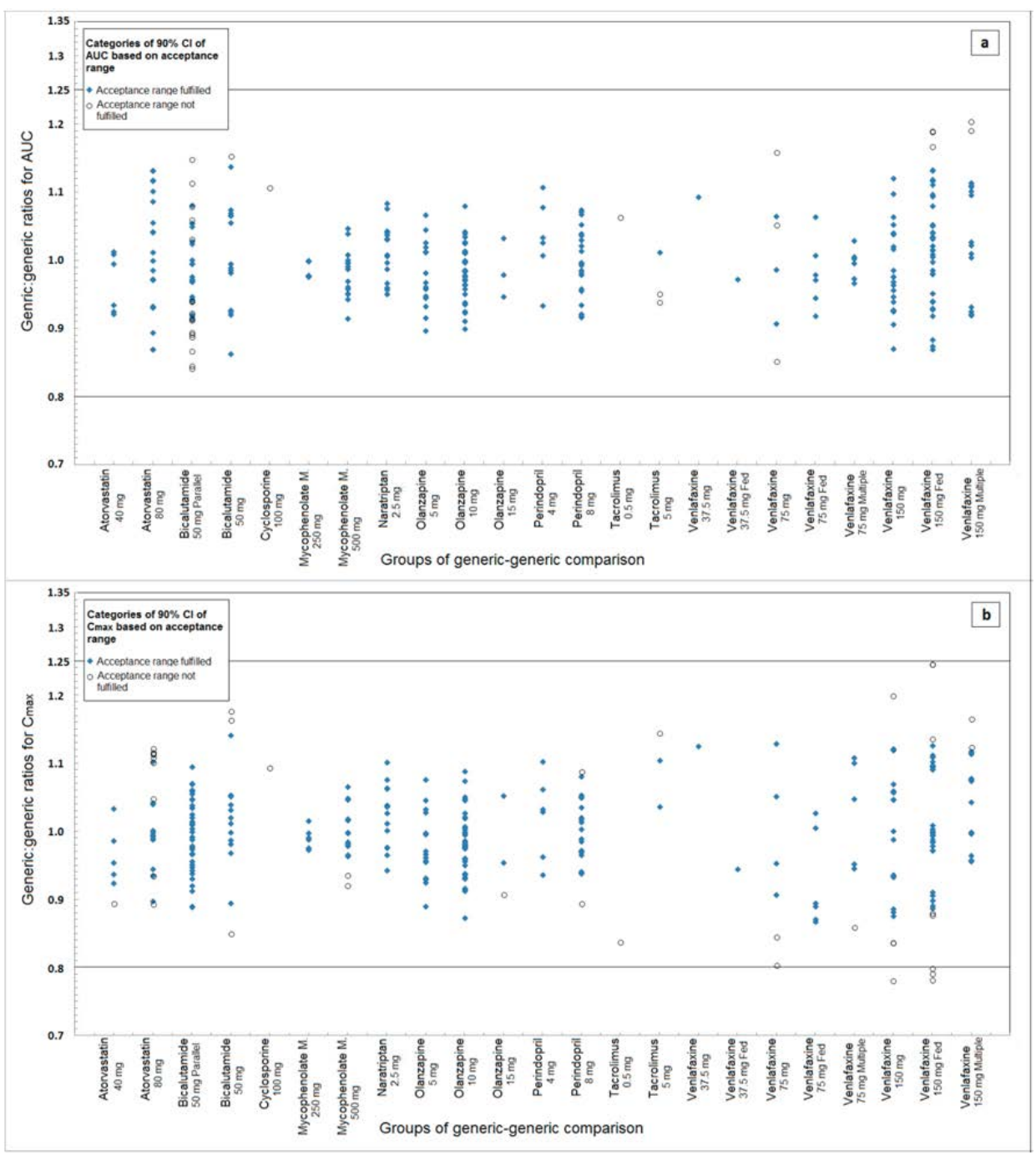

Figure 1. Calculated ratios of generic-generic drug comparisons for (a) $A \cup C$ and (b) $C_{\max }(n=292)$. Legend. Dots in blue represent the generic:generic ratios (Y-axis) at every comparison group (the same design with the same strength of APIs) (X-axis) for which the $90 \% \mathrm{Cls}$ were within the acceptance ranges; Circles in black represent for the ratios of their $90 \% \mathrm{Cl}$ s outside the margin. At the $\mathrm{X}$-axis, the group is labeled by API, strength and the study design if not single dose, fasting or crossover. 


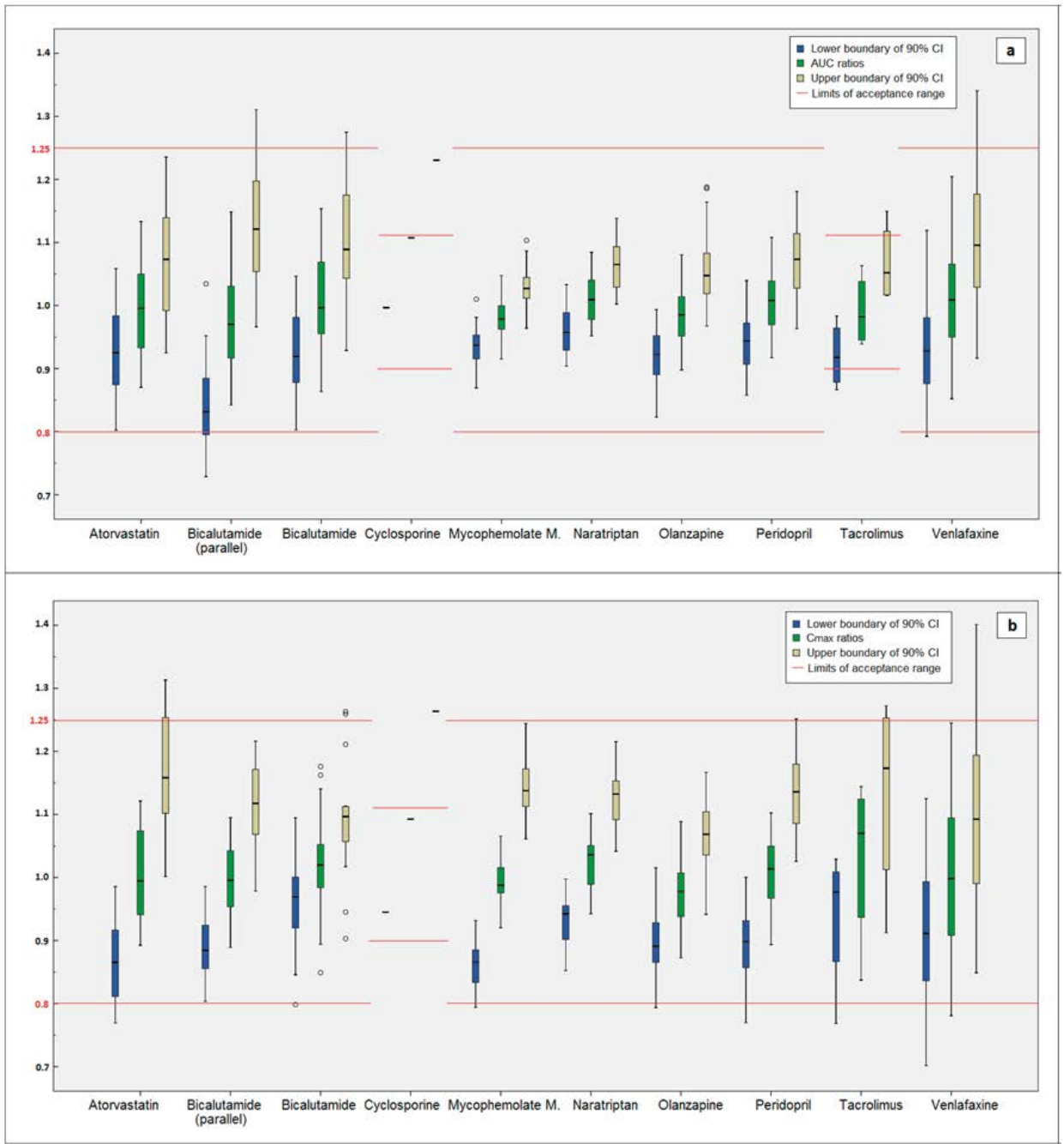

Figure 2. Boxplots of the calculated generic:generic ratios and $90 \% \mathrm{Cls}$ for (a) AUC and (b) $\mathrm{C}_{\max }$ for each API $(n=292)$. Legend. The boxplot in yellow and in blue represents the distribution of upper and lower boundary of 90\% Cls for every API (X-axis), respectively. The boxplot in green represents the ratios. Reference lines (red) represent limits of the acceptance range required by the EMA.

\section{DISCUSSION}

Based on the presented results, bioequivalence between generic drugs can be concluded in $80.5 \%$ of the cases. In $90.1 \%$ of the comparisons, the $90 \% \mathrm{Cl}$ of the ratios was within the $80-125 \%$ (or $90-111 \%$ ) range for AUC and in $87.0 \%$ for $C_{\max }$. Although bioequivalence was not formally demonstrated in $19.5 \%$ of the cases with $90 \%$ Cls outside 
the $80-125 \%$ (or $90-111 \%$ ) margin (either AUC or $C_{\max }$ ), bio-inequivalence, potentially resulting in clinical consequences, was not demonstrated either. In this investigation, the adjusted indirect comparison combines the variability from both bioequivalence studies (Equation 2). Therefore, the comparison results generally yield broader $90 \% \mathrm{Cls}$ for $\mathrm{AUC}$ and $\mathrm{C}_{\max }$ between generic drugs, compared to the $90 \% \mathrm{Cls}$ obtained in actual clinical bioequivalence studies $(9,21)$. According to Glenny et al $(24)$, the SE of the indirect estimate can be expected to be about 1.41 larger than that of the direct estimate. This suggests that when the $90 \% \mathrm{Cls}$ are obtained just outside the $80-125 \%$ (or 90 $111 \%$ ) margin (for instance within a wider range of $75-133 \%$, or $80-125 \%$ ), the actual $90 \% \mathrm{Cls}$ in a direct comparison situation of a bioequivalence study may well be within the acceptance range. Therefore, a failure of showing bioequivalence in our study does not demonstrate that the investigated generic drugs are not bioequivalent. In addition, the study results show that only 3 cases $(1.0 \%, n=292)$ for AUC and 9 cases $(3.1 \%$, $\mathrm{n}=292$ ) for $\mathrm{C}_{\max }$ can be considered as extreme cases, i.e. exceeding the wider $75-133 \%$ (or $80-125 \%$ ) criterion. Although our results cannot fully eliminate uncertainty regarding generic-generic interchangeability, since formal bioequivalence between generics was not demonstrated in all cases, no cases of bio-inequivalence were noted in our comparisons.

\section{Potential clinical relevance of observed differences for $A \cup C$ and $C_{\max }$}

Overall, the generic : generic ratios and $90 \% \mathrm{Cls}_{\text {for }} \mathrm{C}_{\max }$ showed a larger variation than AUC in every API, and more estimations yielded $90 \% \mathrm{Cls}$ outside the acceptance criterion for $C_{\max }$. $C_{\max }$ is generally considered to be less critical than AUC for concluding therapeutic equivalence $(23,25)$. In most cases, the requirement for $C_{\max }$ aims to regulate the safety profile of the generic formulations. It suggests that in most cases clinical consequences are not expected when bioequivalence cannot be demonstrated for $\mathrm{C}_{\max }$ with a relatively small magnitude of difference. For this reason, regulatory authorities (e.g. EMA \& FDA) allow using a widened acceptance range for $C_{\max }$ for highly-variable drugs $(25,26)$. Specific API-related issues on the clinical relevance of $90 \% \mathrm{Cls}$ outside the $80-125 \%$ margin (see Table 2 ) are discussed below.

\section{Atorvastatin}

For atorvastatin, deviations from the $80-125 \%$ criterion were only seen for $\mathrm{C}_{\max }$ (Table 2). Bio-inequivalence was not indicated in our comparisons. As discussed above, atorvastatin is a well-known highly variable drug (i.e. intra-subject variability $>30 \%$ ), so a widened acceptance range (75-133\%) may be used (27). Although the intrasubject variability is not known for the specific bioequivalence studies, this possibility suggests that exceeding of the $80-125 \%$ may not be clinically relevant per se. Further, the magnitude of all exceeded cases was relatively small, i.e. within the range of $75-133 \%$, which supports that these cases are unlikely to be clinically relevant. In the literature, generic 
atorvastatin products have been shown to have comparable treatment outcomes with the innovator drug and were well-tolerated (28), and no significant differences in efficacy, adverse events and patient management were seen upon clinical substitution (29, 30).

\section{Bicalutamide}

The elimination half-life of bicalutamide is very long (5-6 days) (31). In patients, bicalutamide is given daily (50 mg tablet), after which an increase of the plasma concentration by 10 -fold occurs as a consequence of the long half-life. As a result, the difference in $\mathrm{C}_{\max }$ in single dose exposure between the generics is expected to be limited in actual clinical treatments. In addition, the 16 cases of $90 \%$ Cls for AUC outside the $80-125 \%$ margin appeared only in the comparisons of parallel studies. As the $90 \% \mathrm{Cls}$ are wider in a parallel design study due to intersubject variability, the likelihood of obtaining such $90 \% \mathrm{Cls}$ outside the margin is higher for this type of study. Further, despite this increased likelihood, the magnitude of all exceeded cases was relatively small, i.e., within the range of $75-133 \%$, which suggests that these cases may not be clinically relevant.

\section{Cyclosporine}

For cyclosporine, both generic formulations were registered based on the $80-125 \%$ criterion, because they were registered before the EMA requirements on narrowed acceptance ranges for this drug (23) came into force. However, one of the two bioequivalence studies as support for registration of the cyclosporine generics actually fulfilled the $90-111 \%$ range. It is obvious that the indirect comparison cannot meet the tightened acceptance range of $90-111 \%$.

The exceeded case is noted in Table 2, where the $90 \% \mathrm{CI}$ for AUC was within a wider range of $80-125 \%$, but the $C_{\max }$ (94.5-126.5\%) exceeded the margin. The adverse events of cyclosporine were screened in the database of The Netherlands Pharmacovigilance Centre Lareb, and no potential events related to formulation switching were found. However, it is acknowledged that pharmacovigilance databases like the one from Lareb suffer from under-reporting. Also the fact that generic-generic exchange is not expected to occur regularly for this drug may add to the lack of reports regarding the issues for generic cyclosporine drugs. Thus, although bio-inequivalence is not indicated by our results, it is uncertain if the deviation from the $90 \%$ acceptance criteria for cyclosporine leads to a different benefit-risk in the clinic.

\section{Tacrolimus}

Due to a long and variable elimination half-life of $11-16$ hours in the patients $(23,32)$, an accumulation of tacrolimus concentration would be expected in treatment and thus a difference in single dose $C_{\text {max }}$ level is not expected to clinically impact the treatment. According to other literature (33-36), therapeutic equivalence between the innovator and generic tacrolimus has been observed without safety concerns. In clinical practice, 
dose titration is required for tacrolimus treatment in organ transplant patients. Therefore, therapeutic equivalence between different generic tacrolimus drugs is also likely to be the case under such dose titration, and clinical consequences due to our observed minor exceeding in AUC over the $90-111 \%$ limits based on the estimation (Table 2 ) are not expected.

\section{Venlafaxine}

As venlafaxine is an extended release formulation, the pharmacological properties are more sensitive to limited differences in the generic drugs compared with immediate release formulation. This could lead to widespread $90 \% \mathrm{Cls}$ upon generic-generic drug substitution of venlafaxine. The exceeded cases are noted in Table 2, in which one case for AUC and seven cases for $C_{\max }$ exceeded a wider range of $75-133 \%$, which indeed indicates an uncertainty related to clinical consequences. However, for extended release formulations, the demonstration of bioequivalence under three conditions are required by EMA, i.e. single dose fasting and fed and steady state $(2,22,37)$. In our study, for most comparisons between generic drugs, bioequivalence under only one of the requested conditions could not be demonstrated. This situation is quite different from the immediate release formulation where only one pivotal bioequivalence study is requested. Since in all cases, bioequivalence is demonstrated under difference situations, the probability of inequivalence under only one condition affecting clinical outcomes is expected to be limited. Of note, FDA requires only bioequivalence under single-dose fed condition for the registration of venlafaxine generic drugs, thus the situation of interchangeability for venlafaxine generic drugs in the US can be different from Europe (38). In literature, bio-inequivalence in fasted state for venlafaxine $75 \mathrm{mg}$ has been reported between the generic and the innovator drug approved by FDA (39).

In this study, an initial cohort of APIs was defined based on the registration date of generic drugs, which was considered to be independent of bias, for instance potential difficulties of demonstration of bioequivalence. The initial cohort contained a large number of APIs $(n=21)$, allowing a valid selection from the cohort. The selected 6 APIs from the initial cohort are not considered to be the easy cases in term of the demonstration of bioequivalence, for instance, atorvastatin, bicalutamide and venlafaxine. A selection bias (except for the immunosuppressants that were included in the study) is not expected. Furthermore, the selected APIs had large numbers of generic drugs, e.g. atorvastatin (18 generic brands), bicalutamide (18), olanzapine (20) and venlafaxine (19) (Table 1). Although the selected APIs may not be the APIs with the largest number of generic drugs, they can still be considered to be representative to a general pattern of registered generic drugs.

The study results are in line with our previous study (9) and comparative bioavailability study (10) for investigation into the generic-generic interchangeability of topiramate and gabapentin. Furthermore, for tacrolimus, our findings are in line with a similar study performed by Herranz et al. (40). Consistently, when considering all 120 bioe- 
quivalence studies in this investigation, the mean absolute deviation of the ratios from $100 \%$ in this set of generics was $4.5 \%$ for $A \cup C$ and $5.1 \%$ for $C_{\max }$. These figures are also comparable with the results of a study from the FDA (5), which showed that the mean absolute deviation from $100 \%$ for $A \cup C$ and $C_{\max }$ being $3.6 \%$ and $4.4 \%$, respectively. In our opinion, strengthened by justified unbiased selection of drugs, it is reasonable to assume that our estimation of generic-generic interchangeability from this study can be generalized as an overall pattern for a larger group of registered generic medicines.

Recently, a retrospective study reported a significant difference in serum level between generic phenytoin drugs and an increased seizure event rate following switching from one to other generic drug in Korean patients (41). However, the possible selection bias was not justified for the study. The patients for whom the records of serum phenytoin levels were available may have been more susceptible for instable treatment effects, resulting in higher risk of seizures. Furthermore, the causal relationship between the generic phenytoin switching and decrease in serum level (and increase in seizure events) cannot be concluded, since it is unclear when the serum phenytoin levels were recorded, what daily dose was administrated and which generic drug was used in the pre- and post-interchange period in every patient. Thus, further research is warranted for the generic drugs involved in that study, and the conclusion may not be extrapolated to other generic drugs. In some papers, it is proposed that for registration, generic drugs should not only be compared to their innovator, but also to other generics that are already on the market. In our opinion, such a request would not be realistic. Further, this would even need to be repeated when another generic is applied for. Based on the outcome of this study, the actual chance of having a $90 \% \mathrm{Cl}$ outside the criteria upon exchange of generics in direct comparisons is expected to be small. However, the possibility of exceeding the $80-125 \%$ margin in the real life conditions cannot be excluded. Of note, the $80-125 \%$ criterion is not directly linked to efficacy or safety. Thus, exceedance of the $80-125 \%$ margin cannot be directly interpreted as resulting in clinical consequences per se.

\section{CONCLUSION}

Based on a conservative approach, our study demonstrates that more than $80 \%$ of the registered generic drugs were not only bioequivalent to the innovator but also to each other. Due to methodological constraints in our comparison, the $90 \% \mathrm{Cls}$ obtained in this study are generally larger than in the actual within-study comparisons. Therefore, we expect that the actual percentage of generics being bioequivalent to other generics in the actual clinical setting will be higher than $80 \%$. However, our results also imply that formal bioequivalence between generics could not be demonstrated in maximally $20 \%$ of the cases. Still, the magnitude by which the $90 \% \mathrm{Cls}$ boundary is exceeded should not be interpreted as the actual difference between generic drugs in clinical 
practice (21), and in the vast majority of the cases the exceedance of the acceptance criteria is limited. Further, although bioequivalence could not be demonstrated in some cases, in none of the cases the reverse, i.e., bio-inequivalence was demonstrated. Thus, although the results are not fully reassuring, we consider a pronounced risk upon generic-generic exchange in clinical practice as unlikely. Overall, our study suggests that exposure-related risks associated with the exchange of different generic drugs in clinical practice is limited, and not much increased -if any- to the situation in which a generic is exchanged with the innovator.

\section{ACKNOWLEDGMENTS}

The study described was supported by the Medicine Evaluation Board (CBG-MEB) in The Netherlands. The opinions in this article are only those of the authors. This article is not intended to reflect the opinion of the Medicines Evaluation Board in the Netherlands nor any of the working parties or scientific committees of the European Medicines Agency.

The authors acknowledge Christine Gispen-de Wied, MD, PhD, at CBG-MEB for research support. No compensation was provided for her role in the study. 


\section{REFERENCES}

(1) Guidance for Industry. Bioavailability and Bioequivalence. Studies for Orally Adminsitered Drug ProductsGeneral Considerations. US Department of Health and Human Services, Food and Drug Administration Center for Drug Evaluation and Research (CDER) (2003).

(2) Guidline on the Investigation of Bioequivalence. (Committee for Medicinal Products for Human use (CHMP), European Medicine Agency,, London, 2010).

(3) Annex7. Multisource (generic) pharmaceutical products: guidelines on registration requirements to establish interchangeability. (World Health Organization, Geneva, 2006).

(4) Guidance Document-Comparative Bioavailability Standards: Formulations Used for Systemic Effects (Health Canada, Canada, 2012).

(5) Davit, B.M. et al. Comparing generic and innovator drugs: a review of 12 years of bioequivalence data from the United States Food and Drug Administration. Ann Pharmacother 43, 1583-97 (2009).

(6) Anderson, S. \& Hauck, W.W. The transitivity of bioequivalence testing: potential for drift. Int J Clin Pharmacol Ther 34, 369-74 (1996).

(7) Karalis, V., Macheras, P. \& Bialer, M. Generic products of antiepileptic drugs: a perspective on bioequivalence, bioavailability, and formulation switches using Monte Carlo simulations. CNS drugs 28 , 69-77 (2014).

(8) Karalis, V., Bialer, M. \& Macheras, P. Quantitative assessment of the switchability of generic products. European journal of pharmaceutical sciences: official journal of the European Federation for Pharmaceutical Sciences 50, 476-83 (2013).

(9) Maliepaard, M., Banishki, N., Gispen-de Wied, C.C., Teerenstra, S. \& Elferink, A.J. Interchangeability of generic anti-epileptic drugs: a quantitative analysis of topiramate and gabapentin. Eur J Clin Pharmacol 67, 1007-16 (2011).

(10) Yu, Y., Teerenstra, S., Vanmolkot, F., Neef, C., Burger, D. \& Maliepaard, M. Interchangeability of gabapentin generic formulations in the Netherlands: a comparative bioavailability study. Clin Pharmacol Ther 94, 519-24 (2013).

(11) Harrison, J.J. et al. Generic immunosuppression in solid organ transplantation: a Canadian perspective. Transplantation 93, 657-65 (2012).

(12) Kovarik, J.M., Noe, A., Wang, Y., Mueller, I., DeNucci, G. \& Schmouder, R.L. Differentiation of innovator versus generic cyclosporine via a drug interaction on sirolimus. Eur J Clin Pharmacol 62, 361-6 (2006).

(13) Hulbert, A.L., Pilch, N.A., Taber, D.J., Chavin, K.D. \& Baliga, P.K. Generic immunosuppression: deciphering the message our patients are receiving. Ann Pharmacother 46, 671-7 (2012).

(14) Cutler, C. et al. Generic immunosuppressants in hematopoietic cell transplantation. Biology of blood and marrow transplantation: journal of the American Society for Blood and Marrow Transplantation 17, $285-$ 90 (2011).

(15) Christians, U. Generic immunosuppressants: the European perspective. Transplantation proceedings 31, 19S-22S (1999).

(16) Christians, U., Klawitter, J. \& Clavijo, C.F. Bioequivalence testing of immunosuppressants: concepts and misconceptions. Kidney international Supplement, S1-7 (2010).

(17) Robertsen, I. et al. Use of Generic Tacrolimus in Elderly Renal Transplant Recipients: Precaution Is Needed. Transplantation, (2014).

(18) Dong, X., Tsong, Y. \& Shen, M. Equivalence tests for interchangeability based on two one-sided probabilities. Journal of biopharmaceutical statistics 24, 1332-48 (2014).

(19) Dong, X. \& Tsong, Y. Equivalence assessment for interchangeability based on two-sided tests. Journal of biopharmaceutical statistics 24, 1312-31 (2014).

(20) Bialer, M. \& Midha, K.K. Generic products of antiepileptic drugs: a perspective on bioequivalence and interchangeability. Epilepsia 51, 941-50 (2010). 
(21) Gwaza, L. et al. Statistical approaches to indirectly compare bioequivalence between generics: a comparison of methodologies employing artemether/lumefantrine 20/120 mg tablets as prequalified by WHO. Eur J Clin Pharmacol 68, 1611-8 (2012).

(22) Note for guidance on modified release oral and trandermal doseage forms: section II (pharmacokinetic and clinical evaluation) CPMP/EWP/280/96 Corr *. (Committee for proprietary medicinal products (CPMP), European Medicine Agency, London, 1999).

(23) Question \& Answers: Positions on specific questions addressed to the pharmacokinetics working party. (Committee for Medicinal Products for Human use (CHMP), European Medicine Agency, London, 2014).

(24) Glenny, A.M. et al. Indirect comparisons of competing interventions. Health technology assessment 9, 1134, iii-iv (2005).

(25) Garcia-Arieta, A. \& Gordon, J. Bioequivalence requirements in the European Union: critical discussion. The AAPS journal 14, 738-48 (2012).

(26) Haidar, S.H. et al. Bioequivalence approaches for highly variable drugs and drug products. Pharmaceutical research 25, 237-41 (2008).

(27) Draft Guidance on Atorvastatin Calcium. (U.S. Food and Drug Administration, 2010).

(28) Kim, S.H. et al. Efficacy and tolerability of a generic and a branded formulation of atorvastatin $20 \mathrm{mg} / \mathrm{d}$ in hypercholesterolemic Korean adults at high risk for cardiovascular disease: a multicenter, prospective, randomized, double-blind, double-dummy clinical trial. Clin Ther 32, 1896-905 (2010).

(29) Jackevicius, C.A., Tu, J.V. \& Krumholz, H.M. Statins: is it safe and effective to use generic "equivalents"? The Canadian journal of cardiology 29, 408-10 (2013).

(30) Rahalkar, A.R., Ban, M.R. \& Hegele, R.A. Clinical equivalence of proprietary and generic atorvastatin in lipid clinic patients. The Canadian journal of cardiology 29, 418-22 (2013).

(31) Summary of Product Characteristics: Bicalutamide 50mg film-coated tablets. <http://www.medicines. org.uk/emc/medicine/22272/spc\#PHARMACOKINETIC_PROPS> (2013).

(32) Summary of Product Characteristics: Prograf 0.5mg, 1mg, 5mg Hard Capsules. <http://www.medicines. org.uk/emc/medicine/11102/SPC/Prograf+0.5mg\%2c+1mg\%2c+5mg+Hard+Capsules/> (2013).

(33) McDevitt-Potter, L.M., Sadaka, B., Tichy, E.M., Rogers, C.C. \& Gabardi, S. A multicenter experience with generic tacrolimus conversion. Transplantation 92, 653-7 (2011).

(34) Staffan Rosenborg, A.N.m., Tora Almquist, Lars Wennberg, Peter Bárány. Systematic conversion to generic tacrolimus in stable kidney transplant recipients. clin Kidney J 7, 151-5 (2014).

(35) Heavner, M.S., Tichy, E.M., Yazdi, M., Formica, R.N., Jr., Kulkarni, S. \& Emre, S. Clinical outcomes associated with conversion from brand-name to generic tacrolimus in hospitalized kidney transplant recipients. Am J Health Syst Pharm 70, 1507-12 (2013).

(36) Spence, M.M., Nguyen, L.M., Hui, R.L. \& Chan, J. Evaluation of clinical and safety outcomes associated with conversion from brand-name to generic tacrolimus in transplant recipients enrolled in an integrated health care system. Pharmacotherapy 32, 981-7 (2012).

(37) Guideline on the pharmacokinetic and clinical evaluation of modified release dosage forms (EMA/CPMP/EWP/280/96 Corr1) Draft XXIII. (Committee for Medicinal Products for Human use (CHMP), European Medicine Agency, London, 2013).

(38) Draft Guidance on Venlafaxine Hydrochloride. (U.S. Food and Drug Administration, 2010).

(39) Wright, C.W., Aikman, M.S., Werts, E., Seabolt, J. \& Haeusler, J.M. Bioequivalence of single and multiple doses of venlafaxine extended-release tablets and capsules in the fasted and fed states: four open-label, randomized crossover trials in healthy volunteers. Clin Ther 31, 2722-34 (2009).

(40) Herranz, M. et al. Bioequivalence between generic tacrolimus products marketed in Spain by adjusted indirect comparison. Eur J Clin Pharmacol 69, 1157-62 (2013).

(41) Shin, J.W., Chu, K., Jung, K.H., Lee, S.T., Moon, J. \& Lee, S.K. Switching between phenytoin generics in patients with epilepsy may lead to increased risk of breakthrough seizure: chart analysis and practice recommendations. Int J Clin Pharmacol Ther 52, 1017-22 (2014). 
Supplementary I. The data of the bioequivalence studies for the selected medicines in the investigation.

\begin{tabular}{|c|c|c|c|c|c|c|c|}
\hline \multirow[b]{2}{*}{ Product name } & \multirow[b]{2}{*}{ G code } & \multirow[b]{2}{*}{$\begin{array}{l}\text { Doses } \\
(\mathrm{mg})\end{array}$} & \multirow[b]{2}{*}{$\begin{array}{l}\text { Number of } \\
\text { subjects }\end{array}$} & \multicolumn{2}{|c|}{$90 \% \mathrm{CI}$ for $\mathrm{AUC}$} & \multicolumn{2}{|c|}{$90 \% \mathrm{Cl}$ for $\mathrm{Cmax}$} \\
\hline & & & & $\begin{array}{l}\text { Lower limit } \\
(\%)\end{array}$ & $\begin{array}{l}\text { Upper limit } \\
(\%)\end{array}$ & $\begin{array}{l}\text { Lower limit } \\
(\%)\end{array}$ & $\begin{array}{l}\text { Upper limit } \\
(\%)\end{array}$ \\
\hline \multirow[t]{11}{*}{ Atorvastatine } & AG1 & 40 & 90 & 97.3 & 103.2 & 95.5 & 118.1 \\
\hline & AG2 & 40 & 57 & 97.3 & 107.7 & 97.9 & 123.4 \\
\hline & AG3 & 40 & 81 & 98.0 & 106.0 & 95.0 & 116.0 \\
\hline & AG4 & 40 & 63 & 90.0 & 99.0 & 89.0 & 108.0 \\
\hline & AG5 & 80 & 48 & 96.0 & 114.0 & 89.0 & 115.0 \\
\hline & AG6 & 80 & 112 & 112.2 & 121.5 & 104.6 & 121.0 \\
\hline & AG7 & 80 & 92 & 102.7 & 112.7 & 88.9 & 107.1 \\
\hline & AG8 & 80 & 55 & 94.5 & 109.0 & 88.3 & 114.3 \\
\hline & AG9 & 80 & 93 & 108.7 & 118.6 & 102.7 & 123.6 \\
\hline & AG10 & 80 & 68 & 97.0 & 106.3 & 101.7 & 121.8 \\
\hline & AG11 & 80 & 54 & 109.4 & 121.0 & 92.2 & 120.0 \\
\hline \multirow[t]{17}{*}{ Bicalutamide } & BG1 & 50 & 24 & 92.7 & 101.6 & 95.7 & 100.5 \\
\hline & $B G 2$ & 50 & 42 & 100.5 & 108.2 & 99.6 & 107.1 \\
\hline & BG3 & 50 & 39 & 84.5 & 96.0 & 83.4 & 92.2 \\
\hline & BG4 & 50 & 25 & 96.0 & 112.5 & 95.6 & 108.7 \\
\hline & BG5 & 50 & 30 & 88.0 & 106.0 & 96.5 & 104.6 \\
\hline & BG6 & 50 & 49 & 96.9 & 108.4 & 97.9 & 108.7 \\
\hline & BG7 & 50 & $\mathrm{~T}=30, \mathrm{R}=30$ & 100.3 & 122.8 & 93.1 & 110.4 \\
\hline & BG8 & 50 & $T=24, R=24$ & 85.8 & 102.5 & 91.9 & 107.1 \\
\hline & BG9 & 50 & $\mathrm{~T}=23 ; \mathrm{R}=24$ & 97.6 & 119.7 & 92.6 & 108.9 \\
\hline & BG10 & 50 & $\mathrm{~T}=24 ; \mathrm{R}=25$ & 86.9 & 117.9 & 99.1 & 113.9 \\
\hline & BG11 & 50 & $\mathrm{~T}=23, \mathrm{R}=24$ & 92.0 & 107.0 & 90.0 & 104.0 \\
\hline & BG12 & 50 & $T=24, R=23$ & 87.3 & 111.2 & 91.0 & 111.4 \\
\hline & BG13 & 50 & $R=24, T=24$ & 92.0 & 107.0 & 90.0 & 104.0 \\
\hline & BG14 & 50 & $\mathrm{R}=22, \mathrm{~T}=22$ & 87.3 & 111.2 & 91.0 & 111.4 \\
\hline & BG15 & 50 & $R=34, T=34$ & 91.6 & 113.5 & 92.9 & 109.3 \\
\hline & Excluded* & 150 & $T=33, R=33$ & 97.0 & 124.1 & 100.0 & 121.7 \\
\hline & Excluded* & 150 & 21 & 100.1 & 120.0 & 100.2 & 111.1 \\
\hline \multirow[t]{2}{*}{ Cyclosporine } & CG1 & 100 & 24 & 84.8 & 98.0 & 81.0 & 101.5 \\
\hline & $\mathrm{CG} 2$ & 100 & 24 & 93.0 & 109.0 & 90.0 & 109.0 \\
\hline \multirow{10}{*}{$\begin{array}{l}\text { Mycophenolate } \\
\text { mofetil } \\
\text { (mycophenolic } \\
\text { acid) }\end{array}$} & MG1 & 250 & 33 & 97.6 & 105.7 & 93.0 & 111.9 \\
\hline & MG2 & 250 & 50 & 98.0 & 105.2 & 93.5 & 110.8 \\
\hline & MG3 & 250 & 67 & 98.3 & 103.2 & 92.5 & 109.8 \\
\hline & MG4 & 250 & 36 & 96.6 & 102.3 & 92.2 & 110.1 \\
\hline & MG5 & 500 & 116 & 98.3 & 102.4 & 94.1 & 116.5 \\
\hline & MG6 & 500 & 37 & 101.9 & 108.3 & 89.9 & 107.4 \\
\hline & MG7 & 500 & 82 & 97.2 & 102.7 & 87.7 & 106.0 \\
\hline & MG8 & 500 & 39 & 95.7 & 102.6 & 91.9 & 111.1 \\
\hline & MG9 & 500 & 57 & 92.2 & 100.3 & 94.2 & 107.9 \\
\hline & MG10 & 500 & 33 & 96.0 & 104.0 & 91.0 & 116.0 \\
\hline
\end{tabular}




\begin{tabular}{|c|c|c|c|c|c|c|c|}
\hline \multirow[b]{2}{*}{ Product name } & \multirow[b]{2}{*}{ G code } & \multirow[b]{2}{*}{$\begin{array}{l}\text { Doses } \\
(\mathrm{mg})\end{array}$} & \multirow[b]{2}{*}{$\begin{array}{l}\text { Number of } \\
\text { subjects }\end{array}$} & \multicolumn{2}{|c|}{$90 \% \mathrm{Cl}$ for $\mathrm{AUC}$} & \multicolumn{2}{|c|}{$90 \% \mathrm{Cl}$ for $\mathrm{Cmax}$} \\
\hline & & & & $\begin{array}{l}\text { Lower limit } \\
(\%)\end{array}$ & $\begin{array}{l}\text { Upper limit } \\
(\%)\end{array}$ & $\begin{array}{l}\text { Lower limit } \\
(\%)\end{array}$ & $\begin{array}{l}\text { Upper limit } \\
(\%)\end{array}$ \\
\hline \multirow[t]{6}{*}{ Naratriptan } & NG1 & 2.5 & 28 & 92.8 & 98.6 & 90.0 & 101.8 \\
\hline & NG2 & 2.5 & 35 & 96.4 & 103.5 & 92.8 & 106.1 \\
\hline & NG3 & 2.5 & 25 & 99.6 & 106.6 & 97.4 & 114.2 \\
\hline & NG4 & 2.5 & 26 & 100.0 & 108.0 & 95.0 & 109.0 \\
\hline & NG5 & 2.5 & 30 & 95.3 & 102.4 & 93.2 & 105.9 \\
\hline & NG6 & 2.5 & 26 & 95.6 & 103.9 & 95.7 & 110.9 \\
\hline \multirow[t]{18}{*}{ Olanzapine } & OG1 & 5 & 24 & 86.4 & 105.6 & 89.1 & 106.0 \\
\hline & OG2 & 5 & 24 & 95.6 & 104.4 & 90.5 & 99.2 \\
\hline & OG3 & 5 & 24 & 97.7 & 106.5 & 96.7 & 106.6 \\
\hline & OG4 & 5 & 39 & 92.1 & 101.7 & 88.4 & 99.7 \\
\hline & OG5 & 5 & 40 & 92.4 & 104.3 & 89.5 & 105.0 \\
\hline & OG6 & 5 & 30 & 86.1 & 97.5 & 84.2 & 96.9 \\
\hline & OG7 & 10 & 22 & 97.4 & 104.8 & 98.2 & 110.9 \\
\hline & OG8 & 10 & 24 & 101.2 & 108.2 & 98.6 & 111.1 \\
\hline & OG9 & 10 & 20 & 100.0 & 119.2 & 103.5 & 116.1 \\
\hline & OG10 & 10 & 22 & 99.4 & 105.7 & 96.4 & 108.2 \\
\hline & OG11 & 10 & 22 & 95.5 & 101.2 & 94.7 & 109.8 \\
\hline & OG12 & 10 & 16 & 98.0 & 105.0 & 91.0 & 102.0 \\
\hline & OG13 & 10 & 36 & 96.2 & 103.0 & 99.2 & 109.2 \\
\hline & OG14 & 10 & 35 & 98.6 & 106.1 & 94.0 & 106.4 \\
\hline & OG15 & 10 & 23 & 93.7 & 108.6 & 96.9 & 108.8 \\
\hline & OG16 & 15 & 15 & 99.0 & 110.0 & 91.0 & 117.0 \\
\hline & OG17 & 15 & 17 & 94.7 & 103.6 & 88.4 & 99.3 \\
\hline & OG18 & 15 & 22 & 94.9 & 110.3 & 91.6 & 106.1 \\
\hline \multirow{12}{*}{$\begin{array}{l}\text { Perindopril } \\
\text { tert-butylamine }\end{array}$} & Excluded* & 2 & 35 & 100.2 & 106.8 & 98.8 & 112.8 \\
\hline & PG1 & 4 & 42 & 96.7 & 105.4 & 90.5 & 105.5 \\
\hline & PG2 & 4 & 35 & 90.7 & 98.1 & 94.1 & 107.3 \\
\hline & PG3 & 4 & 35 & 98.0 & 106.0 & 88.0 & 100.0 \\
\hline & PG4 & 4 & 26 & 99.7 & 109.6 & 97.0 & 110.9 \\
\hline & PG5 & 8 & 34 & 94.9 & 102.0 & 97.2 & 114.0 \\
\hline & PG6 & 8 & 40 & 102.3 & 108.7 & 92.9 & 111.4 \\
\hline & PG7 & 8 & 30 & 96.5 & 106.0 & 95.5 & 116.2 \\
\hline & PG8 & 8 & 25 & 99.2 & 111.6 & 99.0 & 123.6 \\
\hline & PG9 & 8 & 29 & 97.7 & 109.4 & 89.1 & 109.6 \\
\hline & PG10 & 8 & 34 & 100.0 & 110.0 & 99.1 & 115.0 \\
\hline & PG11 & 8 & 28 & 98.2 & 106.2 & 97.4 & 110.6 \\
\hline \multirow[t]{5}{*}{ Tacroliums } & TG1 & 0.5 & 36 & 91.5 & 105.9 & 103.0 & 120.8 \\
\hline & TG2 & 0.5 & 207 & 101.5 & 108.0 & 90.2 & 96.8 \\
\hline & TG3 & 5 & 42 & 99.2 & 110.9 & 91.7 & 111.5 \\
\hline & TG4 & 5 & 141 & 93.1 & 104.7 & 105.6 & 117.9 \\
\hline & TG5 & 5 & 109 & 96.2 & 103.6 & 110.6 & 121.0 \\
\hline
\end{tabular}




\begin{tabular}{|c|c|c|c|c|c|c|c|}
\hline \multirow[b]{2}{*}{ Product name } & \multirow[b]{2}{*}{ G code } & \multirow[b]{2}{*}{$\begin{array}{l}\text { Doses } \\
(\mathrm{mg})\end{array}$} & \multirow[b]{2}{*}{$\begin{array}{l}\text { Number of } \\
\text { subjects }\end{array}$} & \multicolumn{2}{|c|}{$90 \% \mathrm{Cl}$ for $\mathrm{AUC}$} & \multicolumn{2}{|c|}{$90 \% \mathrm{Cl}$ for $\mathrm{Cmax}$} \\
\hline & & & & $\begin{array}{l}\text { Lower limit } \\
\text { (\%) }\end{array}$ & $\begin{array}{l}\text { Upper limit } \\
(\%)\end{array}$ & $\begin{array}{l}\text { Lower limit } \\
(\%)\end{array}$ & $\begin{array}{l}\text { Upper limit } \\
(\%)\end{array}$ \\
\hline \multirow[t]{39}{*}{ Venlafaxine } & VG1 & 37.5 & 32 & 92.2 & 104.4 & 84.6 & 95.0 \\
\hline & VG2 & 37.5 & 34 & 98.7 & 116.9 & 94.7 & 107.5 \\
\hline & VG3 & 75 & 24 & 86.5 & 115.0 & 97.0 & 113.5 \\
\hline & VG4 & 75 & 38 & 110.4 & 121.0 & 112.5 & 124.8 \\
\hline & VG5 & 75 & 35 & 93.0 & 104.4 & 89.8 & 100.8 \\
\hline & VG6 & 75 & 38 & 94.0 & 118.0 & 92.0 & 108.0 \\
\hline & VG7 & 150 & 36 & 106.3 & 119.4 & 109.5 & 121.6 \\
\hline & VG8 & 150 & 36 & 100.7 & 113.3 & 89.8 & 103.7 \\
\hline & VG9 & 150 & 24 & 93.9 & 104.3 & 90.8 & 102.6 \\
\hline & VG10 & 150 & 42 & 102.3 & 115.5 & 96.0 & 108.8 \\
\hline & VG11 & 150 & 36 & 98.3 & 113.0 & 84.2 & 96.3 \\
\hline & VG12 & 150 & 39 & 101.0 & 121.0 & 96.0 & 107.0 \\
\hline & VG13 & 150 & 37 & 98.2 & 109.0 & 103.0 & 114.0 \\
\hline & G1 & 37.5 & 36 & 98.1 & 110.0 & 99.1 & 112.3 \\
\hline & $\mathrm{G} 2$ & 37.5 & 43 & 95.2 & 107.2 & 92.3 & 107.6 \\
\hline & G3 & 75 & 24 & 103.5 & 111.0 & 101.4 & 111.3 \\
\hline & G4 & 75 & 38 & 99.5 & 109.1 & 101.2 & 112.2 \\
\hline & G5 & 75 & 38 & 92.3 & 103.5 & 87.1 & 98.1 \\
\hline & G6 & 75 & 40 & 97.0 & 116.0 & 90.0 & 102.0 \\
\hline & G7 & 150 & 35 & 99.8 & 113.1 & 95.8 & 108.8 \\
\hline & G8 & 150 & 36 & 103.1 & 112.4 & 107.6 & 123.3 \\
\hline & G9 & 150 & 21 & 95.0 & 105.8 & 106.7 & 119.1 \\
\hline & G10 & 150 & 23 & 94.5 & 103.5 & 98.1 & 107.4 \\
\hline & G11 & 150 & 35 & 103.9 & 112.9 & 108.3 & 119.9 \\
\hline & G12 & 150 & 43 & 87.8 & 101.1 & 82.7 & 97.9 \\
\hline & $\mathrm{G} 13$ & 150 & 66 & 107.7 & 116.8 & 96.0 & 108.8 \\
\hline & G14 & 150 & 46 & 105.0 & 119.0 & 106.0 & 119.0 \\
\hline & G15 & 150 & 41 & 102.0 & 118.0 & 103.0 & 122.0 \\
\hline & VssG1 & 75 & 24 & 103.9 & 113.6 & 100.8 & 111.3 \\
\hline & VssG2 & 75 & 22 & 104.0 & 114.9 & 110.7 & 124.6 \\
\hline & VssG3 & 75 & 34 & 98.5 & 113.7 & 95.3 & 106.8 \\
\hline & VssG5 & 150 & 28 & 97.4 & 111.2 & 94.4 & 107.2 \\
\hline & VssG6 & 150 & 36 & 98.8 & 110.7 & 93.4 & 107.8 \\
\hline & VssG7 & 150 & 34 & 90.9 & 102.0 & 85.7 & 107.9 \\
\hline & VssG8 & 150 & 38 & 110.3 & 119.4 & 91.6 & 109.7 \\
\hline & VssG4 & 75 & 30 & 101.0 & 118.0 & 105.0 & 118.0 \\
\hline & VssG9 & 150 & 35 & 111.0 & 122.0 & 109.0 & 118.0 \\
\hline & VssG10 & 150 & 39 & 102.0 & 113.0 & 102.0 & 114.0 \\
\hline & Excluded* & 225 & 34 & 98.0 & 118.9 & 97.0 & 116.4 \\
\hline
\end{tabular}

* Four studies were excluded from the adjusted indirect comparisons. 
Chapter

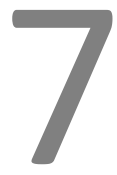

General discussion 

Patients prescribed generic drugs should be able to trust that these drugs are as effective and safe as brand-name drugs or other generic drugs. While the interchangeability of generic and brand-name drugs has been extensively investigated, conclusions are far from unanimous. Despite this, in general, confidence in generic drugs has increased considerably in recent years. However, relatively little is known about the interchangeability of generic drugs. The investigations described in this thesis were mainly based on further analysis of data submitted to support the registration of generic drugs in the Netherlands and provided pharmacological support for the interchangeability of generic drugs with other generic drugs or brand-name drugs.

\section{INTERCHANGEABILITY IN INDIVIDUALS}

In theory, the currently used average bioequivalence approach cannot guarantee that exposure to a generic drug is comparable to exposure to that drug's brand-name equivalent in individual patients. In the past, this concern triggered the investigation of better methods for investigating bioequivalence. Although population bioequivalence and individual bioequivalence approaches were developed as alternatives (1-4) in the last decade, these approaches have not been implemented for several reasons, such as the issues pertaining to a masking effect, power and sample size determination, statistical procedures, and study design $(5,6)$.

With regard to the bioequivalence of a generic drug and its brand-name equivalent, our reanalysis of nine replicate design bioequivalence studies showed that in some individuals drug exposure (measured as AUC and Cmax) was different with the generic drug and the brand-name drug, even though bioequivalence had been demonstrated, based on the $90 \%$ confidence intervals (Cls) of the ratio of the average exposure (for AUC and Cmax) of the generic drug and the brand-name drug being within the acceptance range of 80-125\% (Chapter 3) (7). Interestingly, we also detected the level of drug exposure to be different when brand-name or generic drugs were administered twice in individual participants. We concluded that the variation in drug exposure between the generic drug and the brand-name drug was comparable to that between two administrations of the same drug (either generic or brand-name drug). The variation was predominantly dependent on intrasubject variability, with no additional variation being due to differences in drug formulation. Therefore, with the average bioequivalence approach, exposure to a generic drug, and variations in drug exposure, are expected to be comparable to those of the brand-name drug in individual patients. In another words, the average bioequivalence approach can ensure therapeutic equivalence in individuals, because the observed difference in drug exposure between generic and brand-name drugs is solely due to intrasubject variability. Thus concerns about the appropriateness of the average bioequivalence study are unfounded, and the current 
average bioequivalence approach does not create bioequivalence problems in individual patients.

Nevertheless, in individual patients there remains the possibility that drug exposure will differ if a brand-name drug is switched to a generic drug or if the same drug is administered repeatedly. This makes it important to investigate which pharmacokinetic parameters/variables affect the likelihood that drug exposure will be markedly different after repeated dosing of the same drug. To this end, a modeling study using the data of bioequivalence studies was planned.

\title{
EVALUATION OF THE POSSIBLE IMPACT OF CHANGED REGULATIONS ON THE BIOEQUIVALENCE OF GENERIC DRUGS
}

\author{
Potential cumulative effect of post-marketing quality variations on \\ bioequivalence of a generic drug
}

In the lifetime of a medicinal product, variations in quality are assessed in an independent fashion from case to case. Theoretically, the medicinal product may undergo numerous, non-consecutive post-marketing modifications, such that the ultimate medicinal product may be different from the product at the moment of registration and which gained marketing approval. While the effect of single variations on bioequivalence may be considered negligible in assessments, in the case of multiple quality variations it can be questioned whether the bioequivalence demonstrated between the generic and the brand-name drug at the moment of registration is still applicable for the latest version of the generic drug. In order to investigate whether quality variations in generic drugs have a cumulative effect, necessitating re-evaluation of bioequivalence with the brandname drug, we analyzed post-marketing quality variations in 10 active substances (poorly soluble drugs) (Chapter 4), using a risk-based model that incorporated all critical variations.

With our model, the cumulative effect of critical quality variations was found to affect only a limited number of generic and brand-name drugs (20\%). The quality variations were considered critical if they lead to formulation changes or variations that could potentially influence pharmacokinetic properties. In our dataset, the currently registered generic drugs had, on average, undergone two critical variations. However, it turned out that our model overestimated the risk, based on the validation process. Thus the real risk that multiple quality variations significantly affect the bioequivalence of generic drugs is not known, but it is expected to be less than that estimated with our model. Further optimization and verification of the model is warranted.

On the basis of our findings, we can conclude that the theoretical risk that multiple quality variations to generic drugs influence the bioequivalence with the brand-name 
drug is not a risk in practice for the drugs investigated. This might be because few variations (average of two) have been made to generic drugs in the past decade. However, the risk that multiple quality variations will lead to a re-evaluation of bioequivalence with the latest version of the generic drug increases the longer the drug is on the market (i.e., more quality variations may have been approved), and thus it would be useful to repeat this investigation at a later date, using an optimized model. Although evaluation of the cumulative effect of all post-marketing variations, performed throughout the lifetime of the specific product, is not currently a routine task for regulatory authorities, such evaluations might be useful for monitoring the quality of medicinal products, particularly generic drugs.

\section{Consequences of European Medicines Agency guideline revision}

Regulatory guidelines for medicines need to be modified regularly on the basis of current knowledge, so that they remain up to date. While this is important, it might mean that the bioequivalence of generic drugs varies depending on the requirements of the regulatory guideline used, especially because generic drugs are often registered based on a single bioequivalence study. Thus guideline revision could theoretically affect the bioequivalence demonstrated at the moment of registration. We therefore investigated whether the revision of relevant guidelines affected conclusions regarding the bioequivalence of brand-name and generic drugs, and, if so, what the expected clinical consequences were.

\section{Evaluation of guidance and generic drugs}

To investigate this, we reviewed the current bioequivalence guideline (2010) for immediate-release oral dosage forms of drugs (CPMP/EWP/QWP/1401/98 Rev.1/Corr**) of the European Medicines Agency (EMA)(8), as well as its previous version (2000)(9). Other related guidance documents released from 2001 until the start of this study (2014) were also included. After comparison of the two bioequivalence guidelines and three Pharmacokinetics Working Party (PKWP, preceded by the EWP Therapeutic Subgroup on Pharmacokinetics) Q\&A documents (Box 1), we identified differences in critical requirements that could influence the bioequivalence assessment of generic drugs. Some requirements or product-specific requirements were only available for a restricted period of time, and they were either included in the new Guideline/Q\&A documents or were removed. 
Box 1. EMA guidelines and PKWP Questions \& Answers documents for bioequivalence studies for immediaterelease oral dosage form medicines investigated.

\begin{tabular}{|c|c|c|}
\hline Release date & BE guidelines and $Q \& A$ documents* & Index ${ }^{+}$ \\
\hline July 26, 2001 & $\begin{array}{l}\text { Note for Guidance on the Investigation of Bioavailability and Bioequivalence } \\
\text { (Doc Ref: CPMP/EWP/QWP/1401/98, not available online) }\end{array}$ & NfG 2001 \\
\hline July 27, 2006 & $\begin{array}{l}\text { Questions \& Answers on the bioavailability and bioequivalence guideline } \\
\text { (Doc Ref: EMEA/CHMP/EWP/40326/2006, not available online) }\end{array}$ & Q\&A 2006 \\
\hline January 22, 2009 & $\begin{array}{l}\text { Question \& Answers: Positions on Specific Questions Addressed to the EWP } \\
\text { Therapeutic Subgroup on Pharmacokinetics } \\
\text { (Doc Ref: EMEA/618604/2008, not available online) }\end{array}$ & Q\&A 2009 \\
\hline January 20, 2010 & $\begin{array}{l}\text { Guideline on the Investigation of Bioequivalence } \\
\text { (Doc Ref: CPMP/EMP/QWP/1401/98 Rev. 1/Corr**, } \\
\text { http://wWw.ema.europa.eu/docs/en_GB/document_library/Scientific_guideline } \\
\text { /2010/01/WC500070039.pdf) }\end{array}$ & G BE 2010 \\
\hline July 22, 2010 & $\begin{array}{l}\text { Question \& Answers: Positions on Specific Questions Addressed to the EWP } \\
\text { Therapeutic Subgroup on Pharmacokinetics } \\
\text { (Doc Ref: EMEA/618604/2008 Rev. 2, not available online) }\end{array}$ & Q\&A 2010 \\
\hline
\end{tabular}

\footnotetext{
* The latest version of Q\&A document contains the chapters from previous versions that are not covered by the current guideline in 2010. (Doc Ref: EMEA/618604/2008 Rev.10, http://www.ema.europa.eu/docs/ en_GB/document_library/Scientific_guideline/2009/09/WC500002963.pdf)

†The index is used to indicate guidelines and Question \& Answers documents in the tables and paper.
}

The major differences in critical requirements concerned the acceptance range, acceptance of multiple-dose studies, food intake, analyte (parent compound vs. metabolite), and endogenous substances. In order to determine the effects of these differences in requirements over the years, we selected seven medicines (i.e. active substances) (Table 1) with known pharmacokinetic characteristics (such as, known effect of food on drug availability, highly variable pharmacokinetics, etc) whose bioequivalence could be affected by the above-mentioned differences in requirements. Ninety-two registered generic drugs contain these active substances, and 59 different dossiers were used for the registration of these generic drugs, containing 76 bioequivalence studies. Although 72 studies (95\%) were carried out before the current guideline came into force (i.e., Guideline BE 2010 and/or Q\&A 2010) (Table 2), most ( $n=65,86 \%$ of total number) were compliant with current requirements, representing 48 ( $81 \%$ of total number) dossiers. After re-assessment of the dossiers of older generic drugs according to current guidelines, only $8(14 \%)$ failed to meet current guideline requirements, because of a lack of required studies/data and/or because study findings were negative (i.e., the $90 \% \mathrm{Cls}$ for AUC and/or Cmax exceeded the acceptance range). The results are shown in Figure 1. 
General discussion

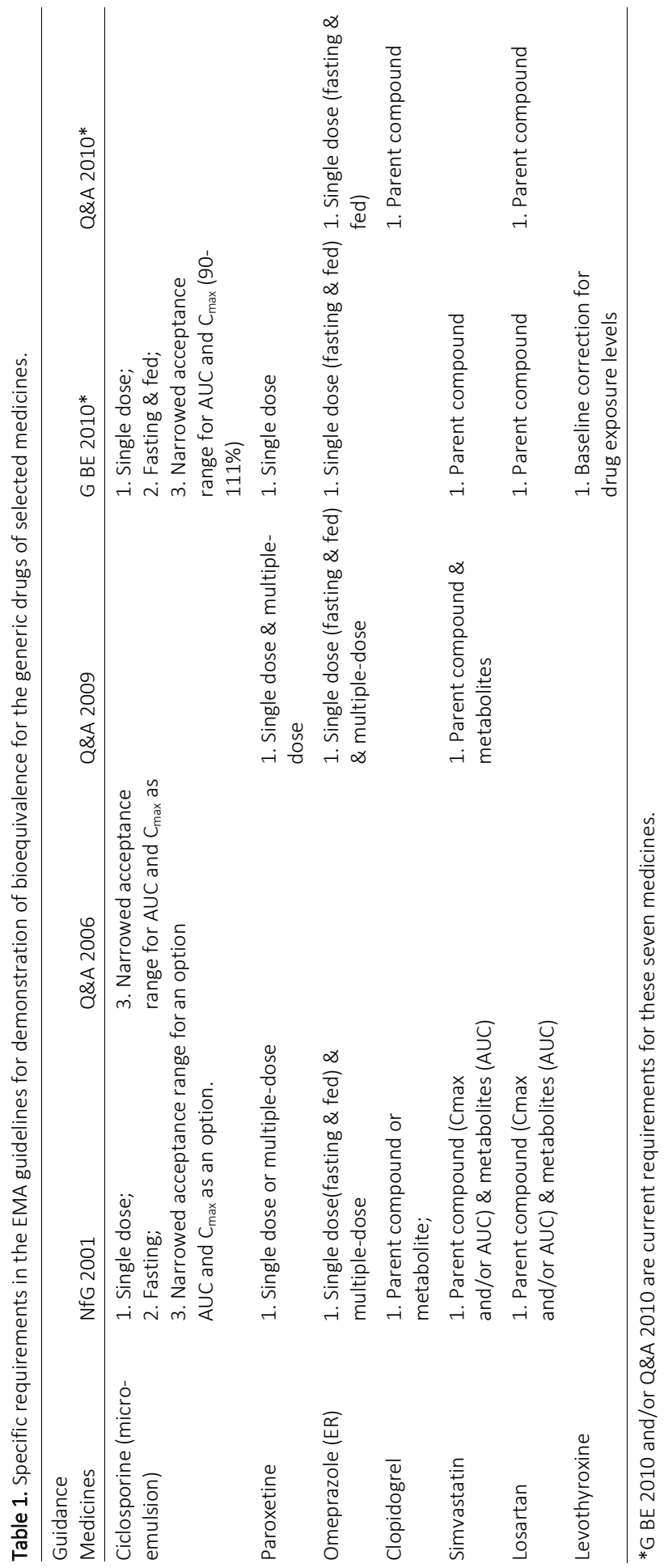


Chapter 7

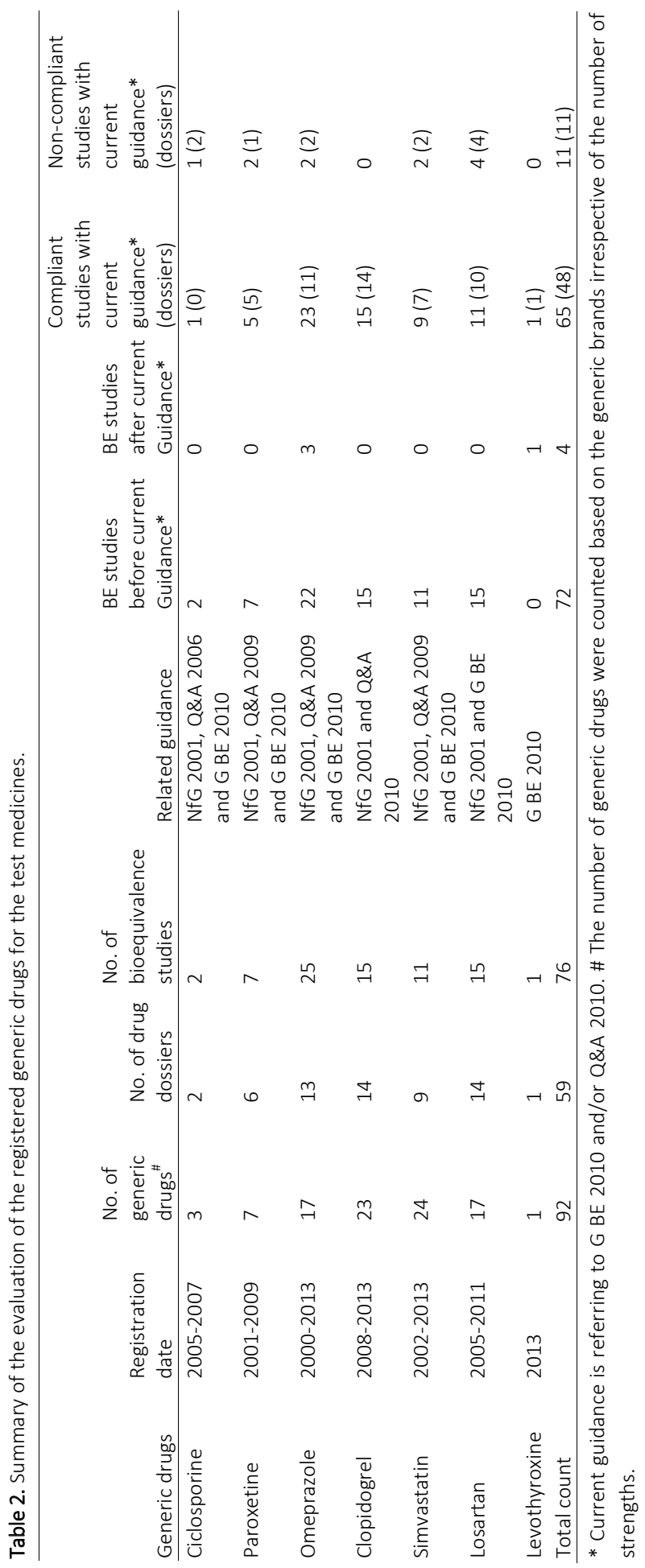




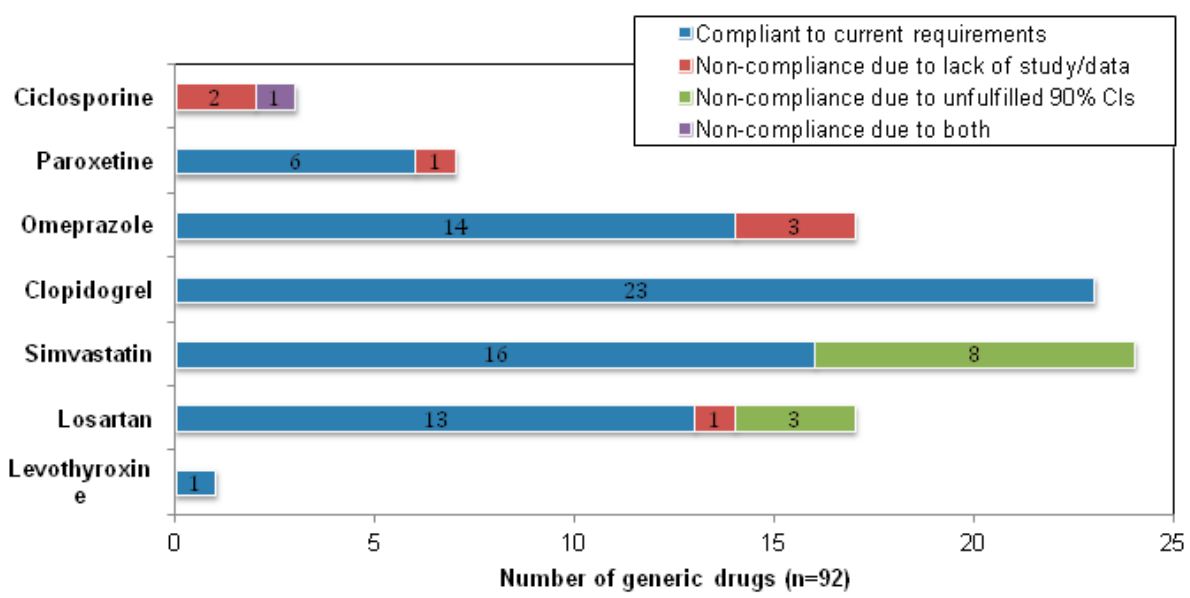

Figure 1. Illustration of evaluation outcome of registered generic drugs for test medicines $(n=92)$.

\section{Bioequivalence acceptance range}

Since 2001, the standard $80-125 \%$ bioequivalence range, by which two treatments are considered not different from each other if the $90 \% \mathrm{CI}$ for AUC and Cmax fall within the range $80-125 \%$, can be extended for highly variable drugs (i.e., intrasubject variability in pharmacokinetics $>30 \%$ ). In the past, the acceptance limits for Cmax for these drugs had been increased to 75-133\%, but nowadays the acceptance range is maximally 70 $144 \%$, depending on the intrasubject variability of the brand-name drug. This means that, in principle, the generic drugs of a highly variable drug might not have demonstrated bioequivalence in the past but might according to current bioequivalence requirements. However, a potential problem arises when the bioequivalence acceptance range is extended, based on the intrasubject variability of the brand-name drug. For drugs that have an intrasubject variability of 30-40\%, the current acceptance range might be tighter than the old fixed $75-133 \%$ range. However, it is highly unlikely that this will occur for generic drugs registered prior to the current guideline. In addition, the current guideline requires evidence that a larger difference in Cmax is not clinically relevant before it will consider a broader bioequivalence range acceptable (10). Thus extending the bioequivalence acceptance range probably has a limited effect.

In contrast, tightening the acceptance range from $80-125 \%$ to $90-111 \%$, as has been implemented by the EMA for cyclosporin and tacrolimus, has implications for the conclusions drawn before this $Q \& A$ document was released that cannot be ignored because the drugs concerned are considered narrow therapeutic index (NTI) drugs. To further investigate the possibility that NTI drugs do not meet current requirements, we investigated the NTI, cyclosporin. We found that one of its two generic drugs did not meet the $90-111 \%$ criterion for AUC and Cmax. Thus concerns about the bioequivalence of NTI drugs following guideline revision appear to be well founded. 
Acceptance of multiple-dose studies, food intake, and analyte

The changes over the years in the requirements for multiple-dose studies, food interaction studies, bioequivalence studies under fed conditions, or analyte could mean that dossiers contain insufficient data if assessed by current standards. However, we thought that this would be unlikely with regard to multiple-dose and food intake studies involving paroxetine, omeprazole, and cyclosporin. One generic paroxetine drug was registered on the basis of two multiple-dose bioequivalence studies (for different strengths), but there was no single-dose study, which is currently required. A single-dose study is considered the most sensitive design for detecting differences in drug absorption between generic and brand-name drugs. The demonstration of bioequivalence with single-dose administration precludes bio-inequivalence between generic and brand-name drugs with multiple-dose administration (commonly used in practice). The current recommendation for paroxetine, as antidepressant, is daily dosing. Thus, although there was no single-dose study for the generic paroxetine drug, relying on the results of multiple-dose studies was not considered to affect the comparability of the generic drug and the brand-name drug in clinical practice. For omeprazole, one dossier lacked a single-dose study under fed conditions, and the bioequivalence of this generic drug with the brand-name drug was established based on single-dose fasting and multiple-dose fed studies. Another dossier contained single-dose fed and multiple-dose fasting studies, but lacked a single-dose fasting study. The situation is the same as for paroxetine, and thus we expect that generic omeprazole drugs will show bioequivalence with omeprazole (recommended daily dosing for several weeks) in clinical practice.

Neither of the dossiers for the generic cyclosporin drugs contained a bioequivalence study under fed conditions. They did, however, include a study of the effect of food on drug absorption. Food had a smaller effect on the absorption of the generic cyclosporin drugs than on the absorption of the brand-name cyclosporin drug. Thus bioinequivalence under fed conditions is not expected between the generic cyclosporin drugs and the brand-name cyclosporin drug.

With respect to requirements regarding the analyte (parent or metabolite), complete data were available for the currently requested parent compound in the dossiers for simvastatin and clopidogrel, but for losartan one study did not investigate the bioequivalence of the parent compound. Losartan is not a pro-drug, and its active metabolite is formed via an intermediate product and not directly from the parent compound. Thus, the pharmacokinetic data for the active metabolite may not reflect the rate of absorption of the parent compound. The lack of data on the comparability of the parent compound in generic and brand-name drugs raises questions about the bioequivalence of the generic losartan drugs.

\section{Endogenous substances}

We could not investigate the consequences of guideline revision on endogenous substances, (e.g., levothyroxine), because no applications for levothyroxine as endogenous 
substance were made prior to enforcement of the latest requirements. Thus, theoretically, in the case of endogenous substances that were registered on the basis of previous guidelines, there may be insufficient data available to satisfy current bioequivalence requirements.

Overall, the lack of relevant studies/data as a result of guideline revision has created uncertainty about registered generic drugs, so that the real impact on bioequivalence has to be evaluated case by case. In addition, changing the analyte to be studied has not only led to missing data, but also to negative results; for example, the metabolites, but not the parent compound, of generic simvastatin and losartan drugs were found to be bioequivalent with the brand-name metabolite. This may have been because of a power problem of the studies, because the pharmacokinetics of the parent compounds of simvastatin and losartan are more variable than those of their metabolites, and thus a large study population is needed to investigate bioequivalence.

\section{DISCUSSION}

The risk of assuming that old generic drugs are bioequivalent with brand-name drugs after changes in bioequivalence requirements appears to be minor. More than $85 \%$ of the studies of generic drugs would still meet current requirements for demonstrating bioequivalence, even though $95 \%$ of the studies were carried out on the basis of previous guidelines. Tightening of the bioequivalence acceptance range may be relevant for generic NTI drugs. While no real concerns were noted for paroxetine, omeprazole, clopidogrel, and simvastatin, further investigations of generic losartan drugs are necessary because currently required data on the parent drug are not available.

Although our findings cannot be extrapolated to other drugs, the investigation of generic drugs containing seven different active substances enabled us to carry out a broad study of the effects of changes to bioequivalence guidelines regarding the acceptance of multiple-dose studies, food intake, analyte (parent compound vs. metabolite), and endogenous substances. Of note, medicines (immediate release) with a relatively simple pharmacokinetic profile are not influenced by the above-mentioned guideline changes. Thus, the proportion of bioequivalence studies, or the number of generic drugs, that still meet current requirements is likely to be higher than that observed in our analysis of the selected seven active substances.

\section{RECOMMENDATIONS AND CONCLUSIONS}

We identified changes in five aspects of bioequivalence requirements that could influence the registration of generic drugs, i.e., acceptance range, acceptance of multipledose studies, food intake, analyte, and endogenous substances. Of seven medicines 
likely to be affected by these changes, we found that most of the bioequivalence studies/dossiers submitted according to past guidelines would still meet current bioequivalence requirements. In a few cases, data that are currently required for demonstrating bioequivalence (e.g., currently requested studies and failure to demonstrate bioequivalence using the currently requested analyte) were not available. This means that, according to current guidelines, the bioequivalence of these generic drugs can theoretically be doubted. The overall proportion of the bioequivalence studies (or number of generic drugs) that still met current requirements was high and probably represents the worst-case scenario, since changes to guideline bioequivalence requirements are not relevant to a large number of generic drugs. For these reasons, we do not consider it necessary to repeal the bioequivalence assessments of older generic drugs. However, the consequences of guideline revision on the bioequivalence of generic drugs vary from medicine to medicine. It should be noted that generic NTI drugs do not meet current bioequivalence requirements and this requires further investigation.

In our opinion, the potential consequence to registered generic drugs should be taken into account when regulatory guidelines are revised.

\section{INTERCHANGEABILITY OF GENERIC DRUGS}

In clinical practice in the Netherlands, patients can be switched from one generic drug to another, and both regulators and prescribers are concerned about the interchangeability of generic drugs (11-14). The indirect comparison study of Maliepaard et al. did not detect generic drift (generic drugs have bioequivalence with the branded drug but not with each other) with generic gabapentin and topiramate drugs (15). In order to confirm the validity of the method used in this indirect comparison study, we carried out a clinical bioequivalence study with gabapentin (Chapter 5) (16). In this study, the three generic gabapentin drugs used in the study of Maliepaard et al were indeed found to be bioequivalent to each other. The study also validated the indirect comparison method, meaning that indirect interstudy comparison provides a reliable estimation of the interchangeability of generic drugs. In a follow-up study (Chapter 6) (17), we used indirect interstudy comparisons to investigate the interchangeability of a broad range of generic drugs (including 9 medicines, encompassing 354 generic drugs registered based on 120 bioequivalence studies). While in most cases (80.5\%), generic drugs showed bioequivalence, bio-inequivalence (defined as $90 \% \mathrm{Cls}$ for AUC or Cmax outside the 80$125 \%$, or $90-111 \%$ margin), which could have clinical consequences, was not demonstrated in the other $19.5 \%$ of the cases (in most instances, the $\mathrm{Cl}$ was only slightly higher than the cut-off limit). Furthermore, because of methodological constraints (Chapter 6 ) (17), the $90 \% \mathrm{Cl}$ values obtained were generally larger than in the actual within-study comparisons. Therefore, more than $80 \%$ of generic drugs are expected to be bioequivalent with other generic drugs in a clinical setting. In conclusion, the indirect comparison 
approach estimated the worst-case scenario for the interchangeability of generic drugs, and so we do not anticipate that switching from one generic drug to another will give rise to clinical problems.

In addition to those pharmacokinetic studies, we were interested in the opinions of patients and doctors regarding the interchangeability of generic drugs. To this end, we carried out a systematic review of publications from the last 15 years (2000-2015), making use of the Medline, API and Embase databases. A query was designed for article selection preliminary from the databases, and keywords included were, for examples, human medications, comparison, substitutions, generic drugs, interchangeability, oral drug, adherence, acceptance, compliant, public opinions etc. A total of 4289 articles were selected by using the query, excluding duplicates among the three databases. The preliminary selection was validated by using an external group of relevant articles. Currently further selection based on titles are complete, and results in 2518 articles. A validation process for the title based selection is planned to be performed by another investigator. The next step of selection is to check the content of abstracts of the articles. As a result, we expected to have review articles, in vivo bioequivalence studies, epidemiology studies, case reports and commentary articles in our selection. These articles will be summarized to give a general overview for the past 15 years in the field of generic drugs. Depending on the quantity of the articles in the final selection, metaanalysis may be conducted for studies with a specific therapeutic indication.

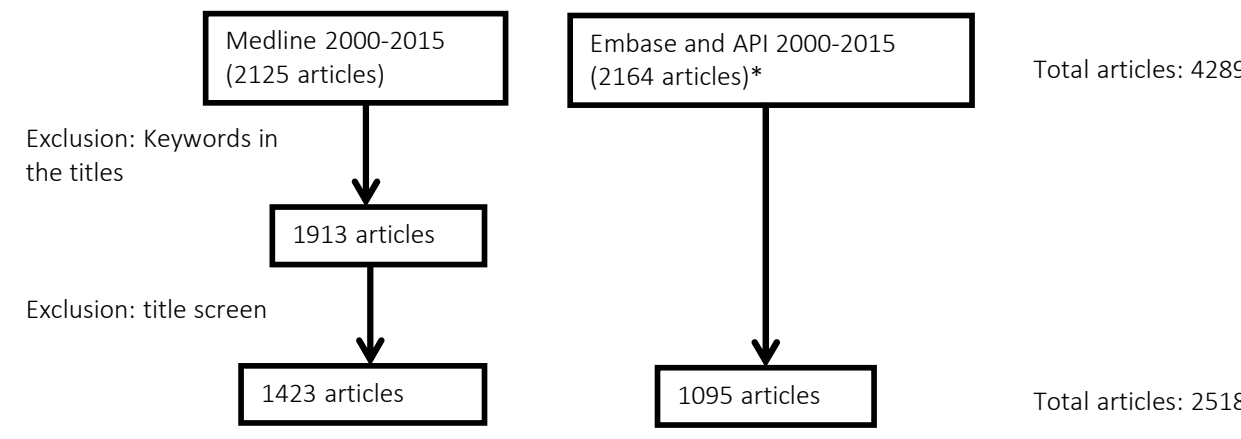

* Duplicate articles have been excluded from the selection of Embase and API based on Medline.

\section{CONCLUSION, A PERSONAL PERSPECTIVE FOR REGULATORY GUIDANCE}

In the studies described in this thesis, we investigated the interchangeability of generic drugs, focusing on individual bioequivalence, the impact of regulatory activities (postmarketing quality variations and guideline revision), and generic-generic drug interchangeability. First, investigation of the intrasubject variability in drug exposure between generic and brand-name drugs showed that differences in exposure when 
switching between generic and brand-name drugs are dependent on intrasubject variability, which in turn is related to the properties of the active substance rather than the formulation. Moreover, the differences in exposure were found to be similar to those seen with repeated administration of generic or brand-name drugs. The difference is not expected to have clinical consequences. It would be interesting to identify which (pharmacokinetic) parameters/variables affect the likelihood of a pronounced difference in drug exposure upon repeated dosing with the same drug.

Regarding regulatory requirements for generic drugs, the results of the study of intrasubject variability confirmed that the current average bioequivalence approach is appropriate for establishing bioequivalence between a generic drug and the brandname drug in individual patients. Furthermore, we did not detect a cumulative effect of multiple post-marketing quality variations on the bioequivalence of registered generic drugs. Although our risk model for the effect of multiple quality variations on generic drugs has not been validated yet (because of overestimation of the effect), this type of investigation should be repeated in the future, because it is anticipated that drugs will be modified with time, as a result of new information and knowledge. Once the impact of multiple quality variations is established, regulatory action will be needed to preclude risks to the interchangeability of generic drugs. Also, revision of the EMA bioequivalence guideline has not affected the bioequivalence of generic drugs that were approved before the current guideline. In other words, the efficacy and safety of previously registered generic drugs are not expected to be different from those of recently approved drugs, even though the drugs were approved on the basis of different regulatory requirements. As guidelines are revised as needed, possible risks regarding the safety and efficacy of registered generic drugs should be considered during further revisions.

The interchangeability of generic drugs was confirmed in a clinical trial of gabapentin and in an indirect interstudy comparison of a broad range of drugs. Current regulations for generic drugs (i.e., demonstration of bioequivalence only with the brand-name drug) do not have consequences for the interchangeability of generic drugs. Such comparisons are useful for medicines with a large number of generic drugs, in order to understand the risks of generic-generic drug switching, and to support regulatory actions. On the basis of our observations, to further ensure generic-generic drug interchangeability, point estimates (i.e., the ratio between generic and the brand-name drug) and intrasubject variability of the brand-name drug could be considered as acceptance criteria, instead of the current bioequivalence acceptance range of $80-125 \%$. It is possible to adjust the 80-125\% acceptance range to the proposed criteria, as the $90 \% \mathrm{Cl}$ values for pharmacokinetic parameters are calculated based on point estimates and the standard (residual) error in ANOVA analysis. We found the residual error to be dependent on intrasubject variability in the pharmacokinetics of the active substance; subject-byformulation interactions did not play a role (7). As the intrasubject variability is a property of the brand-name drug, we only need to establish an acceptance range for the point estimate for the generic drugs containing the same active substance. This range 
for the point estimate can be directly used to check the bioequivalence of one generic drug with another generic drug. Gwaza et al., in their study of WHO bioequivalence data, proposed using the point estimate and study power, concluding that the conditions for demonstrating bioequivalence between generic drugs in indirect comparisons were (a) low point estimate differences between generic drugs $(\leq 5.5 \%)$ for any sufficiently powered study (> 80\%), or (b) larger differences (but less than 14\%) and both bioequivalence studies are overpowered (e.g., 10\% difference and power $\geq 95 \%$ ) (18). The effect of study power is obvious, and as the power calculation is based on the standard error and number of subjects, further research for developing new criteria is warranted, taking the number of subjects into consideration. Further, it would be interesting to know how these factors (i.e., point estimate, standard error, and number of subjects) are correlated and correlated with the $80-125 \%$ criterion.

In conclusion, our investigations provide reassurance that generic drugs are equally effective and safe as brand-name drugs, and that different generic drugs are equivalent to each other, based on current drug regulatory guidelines. Although we did not identify weaknesses in current regulatory guidelines that warrant immediate guideline revision, the issues we considered in the investigation are also relevant for the future, and the methods we developed/validated are appropriate for investigating these issues. We believe that periodic evaluation of registered generic drugs is useful for regulators and also important for the public. To ensure that generic drugs are interchangeable with each other, we recommend using point estimates (i.e., the ratio between generic and the brand-name drug) and the intrasubject variability in drug exposure with the brandname drug as acceptance criteria, instead of the current bioequivalence acceptance range of $80-125 \%$. Of course, this recommendation needs to be substantiated in further studies. 


\section{REFERENCES}

(1) Guidance for Industry: Statistical approaches to establishing bioequivalence. (ed. U.S. Department of Health and Human Services, F.D.A., Center for Drug Evaluation and Research (CDER)) (2001).

(2) Bialer, M. Generic products of antiepileptic drugs (AEDs): is it an issue? Epilepsia 48, 1825-32 (2007).

(3) Guidance for industry: Average, population, and individual approaches to establishing bioequivalence (ed. U.S. Department of Health and Human Services, F.a.D.A., Center for Drug Evaluation and Research (CDER)) (1999).

(4) In Vivo bioequivalence studies based on population and individual bioequivalence approaches. . (ed. U.S. Department of Health and Human Services, F.a.D.A., Center for Drug Evaluation and Research (CDER)) (1997).

(5) Chow, S.-C. Individual bioequivalence-Areview of FDA draft guidance. Drug Information Journal 33, 10 (1999).

(6) Hsuan, F.C. Some statistical considerations on the FDA draft guidance for individual bioequivalence. Statistics in medicine 19, 2879-84 (2000).

(7) Yu, Y., Teerenstra, S., Neef, C., Burger, D. \& Maliepaard, M. A comparison of the intrasubject variation of drug exposure between generic and brand-name drugs: a retrospective analysis of replicate design trials. Br J Clin Pharmacol, (2015).

(8) Guidline on the Investigation of Bioequivalence. (Committee for Medicinal Products for Human use (CHMP), European Medicine Agency,, London, 2010).

(9) Note for Guidance on the Investigation of Bioavailability and Bioequivalence. (Committee for Medicinal Products for Human use (CHMP), European Medicine Agency, London, 2001).

(10) Garcia-Arieta, A. \& Gordon, J. Bioequivalence requirements in the European Union: critical discussion. The AAPS journal 14, 738-48 (2012).

(11) Maliepaard, M. et al. Requirements for generic anti-epileptic medicines: a regulatory perspective. J Neurol 256, 1966-71 (2009).

(12) Anderson, S. \& Hauck, W.W. The transitivity of bioequivalence testing: potential for drift. Int J Clin Pharmacol Ther 34, 369-74 (1996).

(13) Gwaza, L. et al. Statistical approaches to indirectly compare bioequivalence between generics: a comparison of methodologies employing artemether/lumefantrine 20/120 mg tablets as prequalified by WHO. Eur J Clin Pharmacol 68, 1611-8 (2012).

(14) Gagne, J.J., Avorn, J., Shrank, W.H. \& Schneeweiss, S. Refilling and switching of antiepileptic drugs and seizure-related events. Clin Pharmacol Ther 88, 347-53 (2010).

(15) Maliepaard, M., Banishki, N., Gispen-de Wied, C.C., Teerenstra, S. \& Elferink, A.J. Interchangeability of generic anti-epileptic drugs: a quantitative analysis of topiramate and gabapentin. Eur J Clin Pharmacol 67, 1007-16 (2011).

(16) Yu, Y., Teerenstra, S., Vanmolkot, F., Neef, C., Burger, D. \& Maliepaard, M. Interchangeability of gabapentin generic formulations in the Netherlands: a comparative bioavailability study. Clin Pharmacol Ther 94, 519-24 (2013).

(17) Yu, Y., Teerenstra, S., Neef, C., Burger, D. \& Maliepaard, M. Investigation into the interchangeability of generic formulations using immunosuppressants and a broad selection of medicines. Eur J Clin Pharmacol 71, 979-90 (2015).

(18) Gwaza, L. et al. Influence of point estimates and study power of bioequivalence studies on establishing bioequivalence between generics by adjusted indirect comparisons. Eur J Clin Pharmacol 71, 1083-9 (2015). 
Appendix

Summary 

Generic drugs are the drugs that contains the same active substance(s) as the brandname drug, both quantitatively and qualitatively. Bioequivalence between a generic drug and the brand-name drug with respect to the rate and extent of absorption of the active substance(s) has to be demonstrated before generics can be registered. Based on demonstrated bioequivalence, interchange between generic drugs and the brand-name drug is considered not to impact the treatment in terms of effectiveness and safety in clinical practice. Requirements for registration of generic drugs are strict and specifically provided by regulatory authorities worldwide. However, debate regarding the interchangeability of generic drugs has been ongoing continuously for decades, for example on the methodology used to demonstrate bioequivalence and therapeutic equivalence.

In this thesis, we investigated several important aspects of interchangeability of generic drugs that have not been considered or discussed explicitly before, such as the role of intrasubject variability in drug exposure variation after switching from the brandname drug to a generic drug or upon repeating the same drug treatment (Chapter 3 ), the impact of post-marketing quality variations of drugs (Chapter 4), and genericgeneric drug interchangeability (Chapter 5 and 6).

Chapter 1 introduces the history of generic drugs, their legislation, and also the reported concerns regarding interchangeability of generic drugs.

Chapter 2 provides an opinion from regulatory perspectives on generic drug substitutions in general and particularly for the immunosuppressant drugs, i.e. ciclosporine, tacrolimus and mycophenolate mofetil. For registration of a generic drug, the generic drug is requested to demonstrate bioequivalence with the brand-name drug, i.e. performing a single-dose bioequivalence study in healthy volunteers, confirming the same exposure in time, and therefore the same efficacy and safety profile as the brand-name drug. It is well known that the exposure in healthy volunteers may be different than that in patients, due to different comorbidities of the patients. Although this obviously may result in a different absolute exposure in patients, we trust that the relative exposure in healthy volunteers and the patients will be the same for the branded and generic medicine, and bioequivalence demonstrated in healthy volunteers will be valid for the patient population.

Regarding the regulation for the generic ciclosporine, tacrolimus and mycophenolate mofetil, narrowed acceptance range of $90-111 \%$ is requested for $90 \% \mathrm{Cls}$ for AUC and/or Cmax for ciclosprine and tacrolimus considering the properties of narrow therapeutic index. For mycophenolate mofetil, for which bioequivalence is demonstrated based on exposure of the mycophenolic acid metabolite, no narrowing of the criteria was considered necessary by EMA. Doubts about the interchangeability of generic immunosuppressant drugs were discussed in terms of the untested $C_{\text {trough }} / C_{\text {min }}$, multiple dose conditions, and bioequivalence between different generic drugs. First, to our opinion, for an immediate-release product like ciclosporin the PK after the initial absorption from the gastrointestinal tract is essentially governed by the molecular active substance only. Since this substance is identical for the branded and generic ciclosporin formula- 
tions, differences in $\mathrm{C}_{\text {trough, }}$ despite comparable AUC and $\mathrm{Cmax}$ in the case of demonstrated bioequivalence, will be an extremely unlikely event. Second, in clinical practice steady-state conditions are important, the pharmacokinetics of a medicinal product after absorption however is only determined by the active substance, therefore there is no reason to assume that the PK behavior will be different for an immediate-release generic drug product compared with the brand-name drug under steady-state conditions, when a comparable absorption has been demonstrated under the most sensitive condition, i.e., after single-dose administration. Lastly, regarding generic-generic substitution, based on literature data, the occurrence of great differences in exposure upon generic-generic substitution seems unlikely, though formally it cannot be excluded. To further address the concerns on generic-generic substitution, further research has been conducted (i.e. described in Chapter 5 and 6 of this thesis). In conclusion, from a regulatory point of view, generic immunosuppressants like ciclosporin, tacrolimus and mycophenolate mofetil are considered interchangeable with their brand-name drugs.

In clinical practice, differences in drug exposure observed in individual patients upon switching from brand-name to generic drug and triggers concerns or doubt about the therapeutic equivalence of brand-name and generic drugs. Chapter 3 investigates whether differences in total and peak drug exposure upon generic substitution are due to differences between formulations or to intrasubject pharmacokinetic variability of the active substance. For this investigation, 9 replicate design bioequivalence studies retrieved from the database of the Dutch regulatory authority (the Medicines Evaluation Board) that were used for the registration of 7 different generic drugs, i.e. for alendronate, atorvastatin, cyclosporin, ebastine, exemestane, mycophenolate mofetil and ropinirole were reanalysed. Reanalysis was performed with respect to intrasubject variability in total and peak drug exposure (i.e. AUC and Cmax) for both generic and the brand-name drug and also the variance related to the subject-by-formulation interaction.

Results of this reanalysis was that in majority of studies, the intrasubject variability in total and peak drug exposure was comparable for the brand name and generic drugs. It indicates that the variability in drug exposure is not affected by the formulation. This is also confirmed by the results of the variance of subject-by-formulation interaction, which could be considered negligible in our studies. Furthermore, the variability upon switching between the generic and the brand-name drugs was also comparable with that observed for generic and in brand-name drugs after repeated administrations. It means that the variation in drug exposure upon switching between generic and brandname drugs is the same as when repeating the same drug (either generic drug or the brand-name drug). Therefore, based on this investigation, we confirmed that intrasubject variability plays a crucial and decisive role in the variation in drug exposure seen. No additional formulation-dependent variation in drug exposure was observed upon drug switching. The observed differences in exposure that has often been reported or hypothesized in literature upon switching from brand name to generic drug is therefore 
not the result of differences between this brand-name and generic drug, but due to the intrasubject variability in exposure of the active substance.

In Chapter 4, post-marketing quality variations for drugs were investigated with respect to the impact on the interchangeability of generic drugs. We investigated the possibility of a cumulative effect of multiple quality variations applied to both generic and the brand-name drugs, as single variations are assessed by the regulatory authorities on a case by case basis, so per individual variation. For this investigation, we selected the medicines that have poor solubility and/or poor permeability (BCS class II or IV) that have multiple generic drugs available in the Netherlands. For the selected drugs, all quality variations for the brand-name and generic drugs were retrieved from the internal database of the Medicines Evaluation Board. In order to estimate an accumulative effect of different variations on exposure, we built a risk-assessment model that only applied to pre-defined critical variations in our dataset. Compared to a pre-defined threshold, we found only $17 \%$ drugs (13\% generic drugs and $4 \%$ brand-name drugs, $\mathrm{n}=115$ ) had a total risk score above the score that was considered to predict a significant change of the current drug products compared with the drug products at the moment of marketing authorization application. When this cut-off was passed, the current drug product is considered different from the original one, and extrapolation of the demonstrated bioequivalence with the original product may not be applicable anymore for the current product. As the bioequivalence of a generic drug to the brand-name drug is only demonstrated at time of the marketing authorization application, based on the model, the interchangeability of the generic drugs in these $17 \%$ drugs may be suboptimal. However, during model validation, the model appeared to overestimate the total risk for the investigated drugs. Based on the validations results, it appears that the number of generic drugs under risk is expected to be much lower than $17 \%$. Of note, the number of critical variations identified per drug was low ( $n=2$ in average), thus our concerns of frequent critical variations leading to significant changes in the long-time registered drug can neither be relieved nor strengthened. To conclude, among the investigated 115 drugs, the accumulative effect of the quality variations is estimated to have risk in only few generic drugs.

Chapter 5 and 6 provide data in an attempt to relief the concerns regarding genericgeneric interchangeability, which are published in public literature. Up till now, there is not many discussion about the generic-generic interchangeability published, despite the fact that switching between different generic drugs happens in patients, particularly when more and more generic drugs are available nowadays. In theory a so-called 'drift' effect can happen potentially leading to failure of demonstration of bioequivalence between generic drugs. Therefore, in order to check this theoretical possibility in practice, we conducted a comparative bioavailability study (Chapter 5) using gabapentin as a test medicine. In this study, drug exposure of gabapentin after single administration of the brand-name drug and three generic drugs currently marketed in the Netherlands was compared. Following the standard requirements of the EMA guideline, the study 
was conducted in a single dose four-way crossover design in 24 healthy volunteers under fasting conditions. The study demonstrated that all three gabapentin generic drugs were bioequivalent to the brand-name drug, and also bioequivalent to each other. Our investigation therefore provides solid evidence to support that drug switching from a gabapentin generic to another generic drug is not expected to affect the treatment. Of note, selection of gabapentin as a test drug for this study was based on a previous interstudy comparison of drug exposure between generic drugs (published in Eur J Clin Pharmacol 67, 1007-16 (2011)). Also in this preceding investigation, bioequivalence of different gabapentin generics was predicted. The results of in vivo bioequivalence study were therefore in line with previous indirect comparison for gabapentin generic drugs, which validates the method used for such indirect inter-study comparison. In Chapter 6, we used the validated indirect inter-study comparison method to investigate the bioequivalence between a broad range of generic drugs, encompassing 120 bioequivalence studies. The medicines included in this investigation are atorvastatin, bicalutamide, naratriptan, olanzapine, perindopril and venlafaxine and three immunosuppressants i.e. ciclosporin, tacrolimus and mycophenolate mofetil. In total, 292 indirect comparisons between generic drugs with respect to the total $(A \cup C)$ and peak exposure ( $\mathrm{Cmax}$ ) were performed. The indirect comparison results indicated that in the vast majority of cases, i.e., $80.5 \%$, the $90 \% \mathrm{Cls}$ for both AUCt and Cmax fell within the bioequivalence criteria (in $90.1 \%$ and $87.0 \%$ for at least AUCt or Cmax, respectively). In $1 \%$ of the 292 indirect comparison for AUCt and 3\% for Cmax, a wider acceptance range of 75-133\% (or 80$125 \%)$ was exceeded. In none of the cases bio-inequivalence was demonstrated. In the evaluation of the results, it should be born in mind that the applied indirect method is a conservative method, leading to broader $90 \%$ confidence intervals than are obtained in an actual bioequivalence study. Therefore, although the results cannot fully exclude the possibility of non-bioequivalence upon generic-generic exchange, we consider a pronounced risk upon generic-generic exchange in clinical practice unlikely. Overall, our study suggests that exposure-related risks associated with the exchange of different generic drugs in clinical practice is limited, and not much increased -if any- to the situation in which a generic is exchanged with the brand-name drug.

In Chapter 7, a general discussion for the research topics in previous chapters is provided and also preliminary results of an on-going systematic review for the opinions from the public domain and the issues from clinics regarding the interchange between generic drugs are presented. Publications from the past 15 years (i.e. 2000-2015) were selected from the Medline, API and Embase databases according to a pre-defined query. In total of 4289 selected articles, further selection based on titles results in 2518 articles. This study is still ongoing and we expected to perform the review based on review articles, in vivo bioequivalence studies, epidemiology studies, case reports and commentary articles.

In addition, we discussed the possible impact of changes in regulations and evaluate if there is any consequence of the revision of bioequivalence guidelines on the generic- 
generic interchangeability on already registered drugs. For this purpose, we identified the differences in critical requirements between the past and current EMA scientific guidelines that potentially may have consequence for demonstration of bioequivalence in registered generic drugs. The possible consequences of the critical changes were evaluated in a selection of 7 drugs as test medicines. The major changes of bioequivalence requirements on five aspects were defined with respect to probable impacts on the registration of generic drugs, i.e. acceptance range, acceptance of multiple-dose studies, food intake, analyte and endogenous substances. For the 7 selected medicines, ninety-two (92) registered generic drugs were identified in the internal database of Dutch regulatory authority (Medicines Evaluation Board). Although 95\% of the supporting bioequivalence studies (72 out of 76) for these drugs were conducted before the most recent guidance was in force, the majority of bioequivalence studies $(86 \%, n=65)$ is also compliant with these current requirements. Only in a few cases, currently requested studies are lacking and bioequivalence cannot be assessed using the latest requirements (e.g. because the metabolite was measured instead of the parent) Although for these drugs a level of uncertainty on the demonstrated bioequivalence exists, repealing of established bioequivalence between the generic and brand-name drugs is not considered necessary.

Investigation of generic drugs is still ongoing. Better understanding of the applied methodology in the demonstration of bioequivalence for generic drugs and of the formulation of the drugs are necessary for improving the regulation. It's not only to strengthen the regulatory requirements, but also to abandon the unnecessary criteria based on robust evidence. In this thesis, the investigations were conducted mainly based on the experiences from regulators, who have a global view of registration of generic drugs, the impacts of current regulation for generic drugs and possible concerns regarding the interchangeability of generic drugs. The methods used in the investigation of generic-generic interchangeability (Chapter 6) has been validated and are applicable for evaluating of interchangeability of the generic drugs that have not been tested in our studies. To certain extend, it can be a tool to provide pharmacokinetic explanations for particular generic drugs that encounter clinical issues with switching generic medicinal products. In conclusion, interchanges of generic to the brand-name drugs or generic to other generic drugs can be applied in clinical practice without clinical consequences. Current regulation for registration of a generic drug in Europe is reasonably strict to ensure the bioequivalence of the generic drug with the brand-name drug. However, there are still rooms to improve the regulations, for example to update the bioequivalence acceptance criterion of $80-125 \%$. 



\section{Samenvatting}

Generieke geneesmiddelen zijn geneesmiddelen die dezelfde werkzame stoffen hebben als het merkgeneesmiddel, zowel kwantitatief als kwalitatief. Voordat een generiek geneesmiddel kan worden geregistreerd moet bio-equivalentie worden aangetoond. Dat wil zeggen dat tussen een generiek geneesmiddel en het merkgeneesmiddel met betrekking tot de snelheid en mate van absorptie van de werkzame stoffen moet worden aangetoond dat deze gelijk zijn. Wanneer bio-equivalentie is aangetoond, kunnen zowel het generieke geneesmiddel als het merkgeneesmiddel gebruikt worden, omdat het geen impact zal hebben op de behandeling voor wat betreft de effectiviteit en de veiligheid in de klinische praktijk. Eisen voor de registratie van generieke geneesmiddelen zijn streng en specifiek voor de verschillende regelgevende instanties over de hele wereld. Er is echter al tientallen jaren een debat gaande over de uitwisselbaarheid van generieke geneesmiddelen, bijvoorbeeld over welke methodologie gebruikt moet worden om bio-equivalentie en therapeutische equivalentie aan te tonen.

In dit proefschrift onderzochten we een aantal belangrijke aspecten van de uitwisselbaarheid van generieke geneesmiddelen die nog niet eerder zijn overwogen of besproken, zoals de variabiliteit in geneesmiddelblootstelling wanneer een persoon overschakelt van het merkgeneesmiddel naar een generiek geneesmiddel, ten opzichte van het herhalen van een behandeling met hetzelfde geneesmiddel (hoofdstuk 3), de impact van variaties op kwaliteit wanneer het geneesmiddel eenmaal op de markt is gebracht (hoofdstuk 4) en de uitwisselbaarheid tussen verschillende generieke geneesmiddelen (hoofdstuk 5 en 6).

Hoofdstuk 1 introduceert de geschiedenis van generieke geneesmiddelen, hun wetgeving en ook de gerapporteerde bezorgdheid over de uitwisselbaarheid van generieke geneesmiddelen.

Hoofdstuk 2 geeft een advies voor de regelgeving voor generieke geneesmiddelen in het algemeen en in het bijzonder voor specifieke immunosuppressieve geneesmiddelen, respectievelijk ciclosporine, tacrolimus en mycofenolaatmofetil. Voor de registratie van een generiek geneesmiddel, moet van het generieke geneesmiddel de bioequivalentie worden aangetoond ten opzichte van het merkgeneesmiddel. Dit wordt gedaan door middel van het uitvoeren van een studie, waarbij gezonde vrijwilligers een enkele dosis krijgen toegediend van het generieke geneesmiddel, ter bevestiging van dezelfde blootstelling in de tijd, en dus dezelfde werkzaamheid en veiligheid als het 
merkgeneesmiddel. Het is bekend dat de blootstelling bij gezonde vrijwilligers anders kan zijn dan bij patiënten, doordat patiënten meerdere aandoeningen tegelijk kunnen hebben. Hoewel dit natuurlijk kan resulteren in een andere absolute blootstelling bij patiënten, wordt er vanuit gegaan dat de relatieve blootstelling bij gezonde vrijwilligers en patiënten hetzelfde zal zijn voor de merkproducten en generieke geneesmiddelen, en dat aangetoonde bio-equivalentie bij gezonde vrijwilligers ook geldt voor patiënten.

Voor ciclosprine en tacrolimus is, gezien de smalle therapeutische breedte (het verschil tussen een effectieve dosis en een toxische dosis), de geaccepteerde variatie in dosis verkleind naar 90-111\% met 90\% betrouwbaarheidsintervallen (Cl's)) voor de totale blootstelling (AUC) en / of de maximale concentratie in het lichaam (Cmax). Voor mycofenolaatmofetil wordt de bio-equivalentie aangetoond door middel van het meten van de metaboliet mycofenolzuur en op basis van de resultaten achtte de EMA het niet nodig de criteria aan te passen. Twijfels over de uitwisselbaarheid van generieke immunosuppressieve geneesmiddelen worden besproken wat betreft de ongeteste dalspiegels (Cthrough / Cmin), herhaalde doseringen en bio-equivalentie tussen verschillende generieke geneesmiddelen. Naar onze mening moet bij een product met directe afgifte zoals ciclosporine wordt de farmacokinetiek (PK) na de initiële absorptie vanuit het maagdarmkanaal bepaald door het moleculair actief bestanddeel. De farmacokinetiek is wat er met de stof gebeurt in het lichaam. Doordat het actieve bestanddeel identiek is voor de merkproducten en generieke ciclosporine, zijn verschillen in dalspiegels zeer onwaarschijnlijk, vanwege een vergelijkbare AUC en Cmax. Ten tweede, in de klinische praktijk zijn steady-state omstandigheden belangrijk. De farmacokinetiek van een geneesmiddel na absorptie wordt echter alleen bepaald door de werkzame stof, daarom is er geen reden om aan te nemen dat het PK gedrag anders zal zijn voor het generieke geneesmiddel, waarbij directe afgifte plaats vindt in vergelijking met de het merkgeneesmiddel onder steady-state omstandigheden, wanneer een vergelijkbare absorptie is aangetoond onder de meest gevoelige situatie, dat wil zeggen, na toediening van een enkelvoudige dosis. Tot slot, met betrekking tot generiek-generiek substitutie lijkt het op basis van gegevens uit de literatuur onwaarschijnlijk dat er grote verschillen zijn in blootstelling na enkelvoudige toediening van de merkgeneesmiddel, hoewel dit formeel niet kan worden uitgesloten. Om de bezorgdheid over generiek-generiek substitutie aan te pakken, is verder onderzoek gedaan (beschreven in hoofdstuk 5 en 6 van dit proefschrift). De conclusie van dit deel van het onderzoek is dat, vanuit het oogpunt van regelgeving bezien, generieke immunosuppressiva zoals ciclosporine, tacrolimus en mycofenolaatmofetil beschouwd kunnen worden als uitwisselbaar met hun merkgeneesmiddel.

In de klinische praktijk waargenomen verschillen in geneesmiddelblootstelling bij individuele patiënten na het overschakelen van een merkgeneesmiddelen naar generieke geneesmiddelen zorgen voor twijfel over de therapeutische equivalentie van het merken het generieke geneesmiddel. Hoofdstuk 3 onderzoekt of verschillen in de totale en de maximale blootstelling aan het geneesmiddel van een generieke vervanging te verklaren zijn door verschillen tussen de formuleringen of farmacokinetische variabiliteit van 
de werkzame stof. Voor dit onderzoek zijn negen bio-equivalentiestudies uit de database van het CBG gebruikt. Hierbij ging het om zeven verschillende generieke geneesmiddelen, respectievelijk alendronaat, atorvastatine, ciclosporine, ebastine, exemestane, mycofenolaatmofetil en ropinirol. Deze studies werden opnieuw geanalyseerd met betrekking tot de intra-subject variatie (de variatie bij meerdere doseringen bij dezelfde persoon) voor wat betreft de AUC en Cmax, zowel voor het generieke en het merkgeneesmiddel en ook de variatie met betrekking tot de 'subject-by-formulation' interactie.

De resultaten van deze heranalyse lieten zien dat in de meerderheid van de studies de totale intra-subject-variatie en de piekblootstelling vergelijkbaar was voor het merkgeneesmiddel en generieke geneesmiddelen. Dit is een indicatie dat de variatie in blootstelling niet wordt beïnvloed door de samenstelling van het product. Dit wordt ook bevestigd door de gevonden verwaarloosbare 'subject-by-formulation'. Bovendien is de variatie bij het omschakelen tussen generieke en merkgeneesmiddelen vergelijkbaar met de variatie die wordt waargenomen bij herhaalde toedieningen voor generieke en merkgeneesmiddel. Het betekent dat de variatie in geneesmiddelblootstelling bij omschakelen tussen generieke en merkgeneesmiddel hetzelfde is als bij het herhaald toedienen van hetzelfde geneesmiddel (hetzij een generiek geneesmiddelen of het merkgeneesmiddel). Daarom, op basis van dit onderzoek, wordt bevestigd dat de intrasubject variatie een cruciale en beslissende rol speelt in de variatie in de blootstelling aan het geneesmiddel. Geen extra samenstellings-afhankelijke variatie in de blootstelling van het geneesmiddel werd waargenomen bij wisselen naar een ander geneesmiddel. De gerapporteerde waargenomen verschillen en veronderstelde verschillen in de literatuur bij het wisselen van merkgeneesmiddel naar een generiek geneesmiddel zijn derhalve niet het gevolg van verschillen tussen dit merk- en generieke geneesmiddel, maar het gevolg van de intrasubject variabiliteit in de blootstelling van de werkzame stof.

In hoofdstuk 4 is gekeken naar de gevolgen van kwaliteitsvariaties van een geneesmiddel nadat het op de markt is gebracht op de uitwisselbaarheid van generieke geneesmiddelen. We onderzochten hierbij het cumulatief effect van meerdere enkele kwaliteitsvariaties op zowel generieke als de merkgeneesmiddelen, omdat de autoriteiten enkel naar enkelvoudige variaties kijken. Voor dit onderzoek hebben we gekozen voor geneesmiddelen die niet goed worden geabsorbeerd door het lichaam (BCS klasse II of IV) en waarvoor meerdere generieke geneesmiddelen beschikbaar zijn in Nederland. Voor de geselecteerde geneesmiddelen werden alle kwaliteitsvarianten voor het merkgeneesmiddel en generiek geneesmiddel opgehaald uit de interne database van het CBG. Om een cumulatief effect van verschillende variaties op de blootstelling te schatten, bouwden we een risico-evaluatie model, waarbij van te voren gedefinieerde essentiële variaties werden toegepast op onze dataset. Vergeleken met een vooraf gedefinieerde drempel, vonden we dat slechts $17 \%$ van de geneesmiddelen (13\% generiek en $4 \%$ merkgeneesmiddelen, $n=115$ ) een totaalscore risico boven de score had die wordt beschouwd als een belangrijk verschil tussen het huidige geneesmiddel 
en het geneesmiddel ten tijde van den marktvergunningsaanvraag. Van deze geneesmiddelen kan op basis van dit model dan niet meer met zekerheid worden gesteld dat het huidige geneesmiddel en het generiek geneesmiddel bio-equivalent zijn. Omdat de bio-equivalentie van een generiek geneesmiddel met het merkgeneesmiddel alleen wordt gedemonstreerd op het moment van de aanvraag van een vergunning kan, kan op basis van het model worden gesteld dat uitwisseling voor $17 \%$ van de generieke geneesmiddelen niet optimaal is. Echter, tijdens modelvalidatie bleek het model het totale risico voor de onderzochte geneesmiddelen te overschatten. Op basis van de validatie van de resultaten blijkt dat het aantal risicovolle generieke geneesmiddelen veel lager is dan $17 \%$. Van belang is dat het aantal kritische variaties per geneesmiddel laag was (gemiddeld twee per geneesmiddel). De conclusie dat met het huidige model onze bezorgdheid om mogelijke gevolgen van frequente kritische variaties bij al voor lange tijd geregistreerde geneesmiddelen niet kan worden weggenomen maar ook niet wordt vergroot. Hieruit kan worden geconcludeerd dat van de 115 onderzochte geneesmiddelen, het cumulatieve effect van de kwaliteitsvariaties slechts in enkele gevallen mogelijk zorgt voor een verhoogd risico.

In hoofdstuk 5 en 6 wordt gepoogd de bezorgdheid over generiek-generiek uitwisselbaarheid die in publicaties naar voren komt te verminderen. Tot nu toe is er niet veel discussie over de generiek-generiek uitwisselbaarheid gepubliceerd. Dit ondanks het feit dat het overstappen van een generiek naar een ander generiek geneesmiddel steeds meer gebeurt bij patiënten, doordat tegenwoordig meer en meer generieke geneesmiddelen beschikbaar zijn. In theorie kan een zogeheten 'drift' effect mogelijk leiden tot bio-inequivalentie tussen generieke geneesmiddelen. Om deze theoretische mogelijkheid in de praktijk te controleren, voerden we een studie uit (hoofdstuk 5) met gabapentine als testgeneesmiddel. In deze studie werden blootstelling aan het gabapentine merkgeneesmiddel en drie generieke geneesmiddelen die momenteel in Nederland op de markt zijn vergeleken na enkelvoudige toediening van het geneesmiddel. Dit onderzoek werd volgens de richtsnoeren van de EMA uitgevoerd als een zogeheten 'vierweg crossover design' bij 24 gezonde vrijwilligers in nuchtere toestand. De studie toonde aan dat alle drie de gabapentine generieke geneesmiddelen bioequivalent zijn met het merkgeneesmiddel, alsmede bio-equivalent aan elkaar. Ons onderzoek geeft dan ook solide bewijs dat het niet te verwachten is dat de omschakeling van de ene generieke gabapentine variant naar een andere variant de behandeling zal beïnvloeden. Van belang om te vermelden is dat de selectie van gabapentine voor deze studie gebaseerd was op een eerdere studie die verschillende generieke geneesmiddelen vergeleek (gepubliceerd in Eur J Clin Pharmacol 67, 1007-1016 (2011)). Ook in dit eerdere onderzoek werd al voorspeld dat de verschillende generieke varianten van gabapentine bio-equivalent waren. De resultaten van de in vivo bio-equivalentie studie waren daarmee in lijn met eerdere indirecte vergelijkingen voor generieke varianten van gabapentine, die daarmee ook laten zien dat het valide is om op een indirecte manier de generieke geneesmiddelen te vergelijken. 
In hoofdstuk 6 gebruikten we de gevalideerde indirecte interstudie vergelijkingsmethode om verschillende studies met elkaar te vergelijken, om zodoende de bioequivalentie voor een breed scala van generieke geneesmiddelen te onderzoeken. Hierbij is naar 120 bio-equivalentie studies gekeken. De geneesmiddelen opgenomen in dit onderzoek zijn atorvastatine, bicalutamide, naratriptan, olanzapine, perindopril en venlafaxine en de drie immunosuppressiva ciclosporine, tacrolimus en mycofenolaatmofetil. In totaal werden 292 indirecte vergelijkingen tussen generieke geneesmiddelen met betrekking tot AUC en Cmax uitgevoerd. De resultaten van de indirecte vergelijkingen suggereren dat in de meeste gevallen, d.w.z. 80,5\%, de 90\% betrouwbaarheidsintervallen van zowel de Cmax en AUC binnen de bio-equivalentie criteria vallen. In 90,1\% en 87,0\% was dirt het geval voor enkel de AUC of de Cmax. In 1\% van de 292 indirecte vergelijkingen werd door de AUC een groter acceptatiebereik van 75-133\% (of 80-125\%) overschreden en in $3 \%$ van de vergelijkingen voor Cmax. In geen van de gevallen kon worden aangetoond dat de geneesmiddelen bio-inequivalent waren. Er moet rekening mee gehouden worden dat de toegepaste indirecte vergelijking een conservatieve methode is, die leidt tot een ruimere $90 \%$ betrouwbaarheidsinterval dan verkregen in een echte bioequivalentiestudie. Daarom kan er worden gesteld dat een groot risico bij generiekgeneriek-uitwisseling in de klinische praktijk onwaarschijnlijk is, hoewel het risico niet volledig kunnen worden uitgesloten. Kortom, onze studie suggereert dat blootstellingsgerelateerde risico's die zijn verbonden aan het overschakelen naar een ander generiek geneesmiddel in de klinische praktijk beperkt is en nauwelijks of niet toeneemt als een merkgeneesmiddel wordt vervangen door het generieke geneesmiddel.

In hoofdstuk 7 wordt een algemene discussie gevoerd over de onderzoeksthema's in de vorige hoofdstukken en worden ook de voorlopige resultaten van een lopend systematische review van de adviezen uit het publieke domein besproken. Ook worden de problemen vanuit de klinische praktijk met betrekking tot de uitwisseling tussen generieke geneesmiddelen gepresenteerd. Publicaties van de laatste 15 jaar (dat wil zeggen tussen 2000-2015) werden geselecteerd uit de Medline, API en Embase databases volgens vooraf gedefinieerde selectiecriteria. Op basis van de selectiecriteria werden 4289 publicaties geselecteerd. Verdere selectie op basis van de titels resulteerde in 2518 artikelen. Dit onderzoek is nog bezig en we zullen naar verwachting het onderzoek baseren op review artikelen, in vivo bio-equivalentie studies, epidemiologische studies, casestudies en recensies. Daarnaast wordt de mogelijke impact van de wijzigingen in de regelgeving besproken en evalueren we de mogelijke gevolgen van een herziening van de richtsnoeren voor de generieke-generieke uitwisselbaarheid van reeds geregistreerde geneesmiddelen. Hiervoor is er naar de verschillen gekeken tussen de vroegere eisen en huidige wetenschappelijke richtsnoeren van de EMA, en met name naar mogelijke gevolgen voor het aantonen van bio-equivalentie van reeds geregistreerde en goedgekeurde geneesmiddelen. De mogelijke gevolgen van de veranderingen werden geëvalueerd aan de hand van een selectie van zeven geneesmiddelen. De belangrijkste vijf belangrijkste bio-equivalentie criteria die werden gedefinieerd met betrekking tot 
mogelijke gevolgen voor de registratie van generieke geneesmiddelen zijn acceptatiecriterium, acceptatie van meervoudige doses studies, voedselinname, de werkzame stof en lichaamseigenwerkzame stoffen. Voor de zeven geselecteerde geneesmiddelen waren 92 geregistreerde generieke geneesmiddelen geregistreerd in de interne database van het CBG. Hoewel 95\% van de ondersteunende bio-equivalentiestudies (72 van 76) voor deze geneesmiddelen werden uitgevoerd voordat de meest recente regels werden ingevoerd, voldoen de meeste bio-equivalentiestudies $(86 \%, n=65)$ ook aan de huidige eisen. Slechts in enkele gevallen ontbreken studies die tegenwoordig vereist en kan bio-equivalentie niet worden beoordeeld aan de hand van de nieuwste eisen (bijvoorbeeld omdat de metaboliet werd gemeten in plaats van de werkzame stof zelf). Hoewel voor deze geneesmiddelen een bepaalde mate van onzekerheid bestaat over de bio-equivalentie, wordt het niet nodig geacht de eerdere conclusie met betrekking tot bio-equivalentie tussen het generieke en merkgeneesmiddel in te trekken.

Onderzoek van generieke geneesmiddelen is nog steeds gaande. Beter begrip van de toegepaste methodologie in het aantonen van bio-equivalentie voor generieke geneesmiddelen is nodig voor het verbeteren van de regelgeving. Dit is niet alleen om de wettelijke eisen te verbeteren, maar ook om eventueel onnodige criteria op basis van solide bewijsmateriaal te schrappen. In dit proefschrift werden de onderzoeken voornamelijk gebaseerd op de ervaringen van toezichthouders, die een globaal overzicht hebben van de registratie van generieke geneesmiddelen, de gevolgen van de huidige verordening voor generieke geneesmiddelen en eventuele zorgen over de uitwisselbaarheid van generieke geneesmiddelen. De methodes die we hebben gebruikt voor het testen van uitwisselbaarheid van generieke geneesmiddelen zijn gevalideerd (hoofdstuk 6) en kunnen ook worden toegepast op andere generieke geneesmiddelen. Tot bepaalde hoogte kan het een middel zijn om eventuele farmacokinetische verklaringen te vinden voor het geval dat bepaalde generieke geneesmiddelen in de kliniek problemen opleveren. Samengevat kan er worden geconcludeerd dat generieke geneesmiddelen in het algemeen in de klinische praktijk kunnen worden toegepast zonder ongewenste klinische gevolgen. De huidige regeling voor de registratie van een generiek geneesmiddel in Europa is strikt genoeg om de bio-equivalentie van het generieke geneesmiddel met het merkgeneesmiddel te garanderen. Er is echter nog ruimte om de voorschriften te verbeteren, bijvoorbeeld door de bio-equivalentie aanvaardingscriterium van $80-125 \%$ aan te passen. 
Appendix

Acknowledgements 
In the past six years, I started my first job and finished my PhD study. For that, I would like to thank Christine, Marc, Kees and David first. Without you and your support, neither of those can be achieved. Kees and David, thanks for your guidance during my PhD study, sharing your knowledge and experiences with me. You are always patient with me and gave me a lot of freedom for doing research. I appropriate your guidance and encouragement.

Christine and Marc, you are super important to me. I secretly call Christine as my 'Dutch mother'. Thank you for everything. I like your humor although it did make me nervous at the beginning. You are a very easy going person with all kindness, and you always trust me and give me a lot of freedom. I am very happy to work with you, and I feel I am the lucky girl because of you. Marc, thanks for giving me a super high grade during my internship. It brought me substantial confidence for becoming a clinical pharmacokinetic assessor at MEB. You are so friendly and kind. I learned a lot from you, not only regarding doing research but also being a great person. I am stubborn particularly in doing research. You are always patient and positive, and successfully convince me at the end (K.O.). Christine and Marc, I am super proud about our Chinese Trip with Hugo. It was my pleasure to show you my home country, and to share my lovely family with you.

Bert and Hugo, thanks for your support and encouragement. When I was a master student at UU, one day I encountered you (Bert) on the way to 'Went gebouw' in the morning. I asked for your opinion on working with Christine in the field of regulatory science at MEB. I still clearly remember you told me that working with Christine was fun and regulatory science would be the future. In fact, you were correct for both terms. I used to ask you difficult questions when I am super confused or hesitate to make a decision. You are always positive and give me powerful and super short answers, which are always helpful. Hugo, thanks for your approval and leading us to Health Sciences Authority (HSA) in Singapore and CFDA in China. It was an extraordinary experience for me. It brought my vision to a higher level, and your gentleness and kindness also impressed me, and influenced me positively. It was a pity that we could not find an 'IJsvogel' in China. Maybe next time.

It was a pleasure to work with Peter, Amr, Steven, Floris, Angela and Emily for the thesis. I greatly appreciate your contributions, particularly to my lovely students, Amr and Emily. You both did a great job and completed a project individually. You both are independent and have the talent to own an excellent career. It was a great experience to guide you in your master studies. Amr, I wish you good luck with your own PhD study, and Emily, I wish you have a desirable career. Steven, thank you so much for your help with statistics for all the studies. Without your support, my life could be much more difficult. In addition, I would like to say a warm thank you to Jane, who did almost all proofreading and English language corrections for this thesis. 
Thanks to my assessment committee and defense committee (the corona), i.e. Prof. dr. A. Bast, Dr. M.H.L Christiaans, Prof. dr. T. van Gelder, Prof. dr. R.A.A. Mathôt, Dr. N.M. Tampal, Prof. dr. R. Koopmans, and Prof. dr. G. Haenen.

Thanks to my colleagues at the MEB, especially to the colleagues which I have worked with, i.e. colleagues from FT 1 and 3, FKT and programmabureau Wetenschap. Thanks for the clinical PK team (Martin, Carolien, Marc, Pieter, Jan, Karolina, Michiel, Nadje, Margje, Esther, Rosana and Jeroen), I am very happy to work with you, and thank you all for sharing your knowledge and experiences with me. I am thankful to be a part of the team. In addition, thanks to all my colleagues from the department of clinical pharmacology and from CARIM school in Maastricht University, particularly for MarieClarie, Marc, Frank, and Rob.

Most importantly, I would like to thank my pretty and handsome paranymphs, Yao and Pieter. Thanks for standing with/behind me during my PhD defense, which makes me feel "safe" and confident. Many thanks to my lovely friends in life, May, Christian, Burcu, Daniel, Nina, Marcel, Yao Liu, Zhida Xu, Yue Li, Bincheng Luo, Sujie Cao, Xiawen Chen, Lv Qian, Weiluan Chen, Qizhi Hu, Qinting He, Dominique, Jolanda, Chee-Hong, Mei Ding, Lu Ye, Jiongwei Wang, Wen Liang, Chong Sun, Yuanjie Yu, Rui Yang, Yuan Yuan, Shasha Liu, Jing Sun, Lin Shi, Lily Li, Hui Gao, Jiansheng Zhang and Jin Fang. May and Yao, you both are my buddies. 感谢你们的出现, 和给我带来的无限快乐与陪伴。感谢荷 兰华人生命科学协会（CALN）理事会和荷兰华人工程师协会（VCWI）理事会一 起奉献的伙伴们。能和大家一起做有意义的事情是我在荷兰最大的收获。

感谢在我身边的恩师和前辈们，任忠俞 老师，Bob Hughes 老师，叶露 教授， 叶华 教授, 丁梅 姐, 高慧姐, 史琳 姐, 李玲华姐, 孙静姐, 张建生 部长, 方进 主任, 和各位海智计划的专家们（HOME program, CAST）。谢谢你们一直以来对 我的认可, 帮助, 提携和指引。

最后, 感谢我的家人。谢谢你们对我默默的支持, 和极大的包容。从不质疑 我的决定和挑剔我的过失。感谢我的妈妈，爱我，抚养我，教育我，支持我出国 读书，一直对我的资助和鼓励。感谢伯伯和哥哥，陪伴妈妈和给我一个新的家。 祝我的家人们健康, 快乐! 



\section{List of co-authors}

\section{David Burger}

Dept. of Pharmacy, Radboud University Nijmegen Medical Centre, Nijmegen, The Netherlands

\section{Peter Jongen}

Medicines Evaluation Board, Utrecht, The Netherlands

\section{Hubert G.M. Leufkens}

Utrecht Institute for Pharmaceutical Sciences, Division of Pharmacoepidemiology and Clinical Pharmacology, Utrecht University, Utrecht, The Netherlands

Medicines Evaluation Board, Utrecht, The Netherlands

\section{Amr Makady}

National Health Care Institute, Diemen, The Netherlands

\section{Marc Maliepaard}

Medicines Evaluation Board, Utrecht, The Netherlands

\section{Cees Neef}

Dept. Pharmacology and Toxicology, CARIM, Maastricht University Medical Centre, Maastricht, The Netherlands

Medicines Evaluation Board, Utrecht, The Netherlands

\section{Steven Teerenstra}

Dept. for Health Evidence, section Biostatistics, Radboud university medical centre, Nijmegen, The Netherlands

Medicines Evaluation Board, Utrecht, The Netherlands

\section{Floris Vanmolkot}

Dept. Internal Medicine, Maastricht University Medical Centre, Maastricht, The Netherlands 



\section{List of publications}

Maliepaard, M., Yu, Y. \& Leufkens, H. Equivalence of generic medicines in general and immunosuppressants in particular - a regulatory opinion on switching of ciclosporin, tacrolimus and mycophenolate mofetil. Generics and Biosimilars Initiative Journal 2, 86-90 (2013).

Yu, Y., Teerenstra, S., Neef, C., Burger, D. \& Maliepaard, M. A comparison of the intrasubject variation in drug exposure between generic and brand-name drugs: a retrospective analysis of replicate design trials. Br J Clin Pharmacol 81, 667-78 (2016)

Yu, Y., Teerenstra, S., Vanmolkot, F., Neef, C., Burger, D. \& Maliepaard, M. Interchange ability of gabapentin generic formulations in the Netherlands: a comparative bioavailability study. Clin Pharmacol Ther 94, 519-24 (2013).

Yu, Y., Teerenstra, S., Neef, C., Burger, D. \& Maliepaard, M. Investigation into the interchangeability of generic formulations using immunosuppressants and a broad selection of medicines. Eur J Clin Pharmacol 71, 979-90 (2015).

Not included in this thesis

Gispen-de Wied, C., Stoyanova, V., Yu, Y., Isaac, M., Pani, L. \& de Andres-Trelles, F. The placebo arm in clinical studies for treatment of psychiatric disorders: a regulatory dilemma. European neuropsychopharmacology : the journal of the European College of Neuropsychopharmacology 22, 804-11 (2012).

Yu, Y., and Maliepaard, M. "Interchangeability of Gabapentin Generic Formulations in the Netherlands: Summary of a Comparative Bioavailability Study." (2013). Published electronically December 17, 2013. http://gabi-journal.net/news/interchangeabilityof-gabapentin-generic-formulations-in-the-netherlands-summary-of-a-comparativebioavailability-study.

Yu, Y., and Maliepaard, M. Voor- en nadelen generiek middel en spécialité gelijk. Pharmaceutisch Weekblad PW26 (2016). 



\section{About the author}

Yang Yu (虞洋) was born in March 03, 1985 in Xi'an China, and grew up there. Yang went to UK when she was 18 years old, and completed the GCE A Level in 2005 at Weigh \& Leigh College. In 2005, she moved to the Netherland and received her first degree - Honor Bachelor of Science in Life Science, at Roosevelt Academy (former University College Roosevelt) in Middelburg, the Netherlands. Yang continued the higher education in University Utrecht and was awarded the Utrecht University Excellent Scholarship for two years. In 2010, she received her master degree in Drug Innovation and specialized in Drug Regulatory Sciences' Profile. During her master studies, she did two independent research projects in the field of pharmaco-epidemiology (Identification of Risk Factors for Drug-Induced Liver Injury) and regulatory science (Conditions of Historical-controlled Trials for Anticancer Treatments).

Yang started her first job at the Medicine Evaluation Board (MEB) in 2010 in The Hague. She worked as a project officer in Science Department, and was responsible for drafting MEB Research Program 2011-2015. The Research Program 2011-2015 is still used as a framework for regulatory science in actions at the MEB.

With the supports of the MEB, Yang started her PhD study (part-time) in the field of clinical pharmacology at Maastricht University in 2011 and worked as a (part-time) clinical pharmacokinetics assessor in the department for oncology drug products at MEB. In the meantime she still facilitated the organization of scientific activities in the Science Department of the MEB, and this task is still ongoing. Since 2015, Yang works as a full-time clinical pharmacokinetics assessor at the MEB, and lives in Nieuwegein, the Netherlands. 



\section{Valorisation Addendum}




\section{RELEVANCE}

Generic drugs and the concept of bioequivalence for registration of a generic drug have been used more than 50 years. Discussions with respect of interchangeability (i.e. therapeutic equivalence) of the generic drugs with the brand-name drug are still ongoing. Although the trust of using generic drugs in practice increased in the past 10 to 20 years, some issues and concerns of using generic drugs are still reported in public media and literature. In this dissertation, interchangeability of generic drugs has been investigated with new approaches, and the findings and conclusions are highly relevant to societies worldwide.

\section{REGULATORY SCIENCE AND INNOVATION}

In the light of regulatory science, the dissertation serves the objectives of the subject that "regulatory science is the scientific domain targeting the development of new instruments, standards and methods to optimally facilitate the evaluation of the efficacy, risks and quality of medicinal products. Knowledge development focuses on both new instruments and their application." In Chapter 3, the results showed that the observed difference in drug exposure after switching to a generic drug was related to intrasubject variability of the active substance, rather than formulation-dependent variation in drug exposure. It indirectly demonstrated that the currently required average bioequivalence approach for registration of a generic drug by regulatory authorities is reliable and also sufficiently to ensure the interchangeability of treatment between generic and the brand-name drug in individual patients. The study also contributes to the discussion for replacing the average bioequivalence approach by individual/population bioequivalence approaches, and in fact supports the action of termination by FDA for replacing the traditional average bioequivalence approach. Furthermore, the studies in Chapter 4 and Chapter 7 were conducted with the purpose of reviewing the regulatory system, further understanding the possible influence of regulatory actions on the interchangeability of generic drugs. The study results showed currently the influence is limited, however the situation may to change along with the increase of the number of post-marketing (quality) variations and revision of guidelines in the future. Thus, the methods applied in these studies can be utilized when a regular check for the similar concerns is needed in the future.

In addition, considering the core responsibility of medicine regulatory authorities, i.e. to ensure the effectiveness and safety of the medicines in patients, investigations of generic-generic drug interchangeability (Chapter 5 and 6 ) is highly relevant and has a unique contribution in this field. First, generic-generic drug interchange happens in clinical practice, however currently no legislation is in place to require a demonstration of bioequivalence between a generic drug to the rest of marketed generic drugs during 
its registration process. Over time, when more and more generic drugs on the market, the occurrence of generic-generic drug interchange will be more frequent. Therefore, it is necessary to have an investigation on this potential issue, understanding the possible consequence, in order to provide rational recommendations for regulatory actions.

Overall, this dissertation does not only provide evidence to resolve the regulatory system related concerns of generic drugs from a regulatory point of views, but also the methods can be used in the future to support regulatory actions by the authorities. In addition, regulatory science itself is an innovative discipline/subject. The majority of the methods applied in the investigations of this dissertation are innovative and can be applied later on. To our knowledge, the clinical trial with gabapentin drugs (Chapter 5) was the first study to investigate the interchangeability between generic drugs in vivo.

\section{Social impact (patients and healthy professionals)}

This dissertation has a great impact on society and economics. In clinical practice, patients may hesitate to take medicines when the treatment is changed from the brandname drug to a generic drug, or from the generic drug to other generic drug. Sometimes, doctors also hesitate to prescribe generic drugs. If there is any complaint regarding effectiveness or safety from the patients who switched between generic drugs, the doctors are likely to ascribe the issue to generic drugs. In turn patients are likely to be switched back to the brand-name drug, which increases the costs substantially. This dissertation concluded that the generic drugs can be trusted. The conclusion is expected to have a positive influence on the prescription of generic drugs, to avoid unnecessary worrying.

\section{Economic impact}

Indirectly the financial burden of the healthcare system may be reduced. Generic drugs are much cheaper than the brand-name drugs (10\% of the price of the brand-name drugs). Unnecessary remaining in use of the brand-name drugs brings financial burden to the health system. From that point of view, increasing the trust of using generic drugs can prevent unnecessary switch-back rate, and increase the prescription of generic drugs, which in turn reduce the burden in the health system.

\section{Academic contributions}

In addition, this dissertation has an obvious contribution to the academic world. Four chapters have been published in scientific journals with high impact factors. The publication of Chapter 3 has been reported as news " Overstappen op generiek niet onveilig " in Nederlands Tijdschrift voor Geneeskunde (Dutch Journal of Medicines). Following this piece of news, we were an invited to publish a summary of the article in Pharma- 
ceutisch Weekblad (Journal of the pharmaceutical association), i.e. "Voor- en nadelen generiek middel en spécialité gelijk " (PW26, 2016). When Chapter 5 was published in the journal of Clinical Pharmacology and Therapeutics, in the same issue of the journal the US FDA published a perspectives paper "Confidence in Generic Drug Substitution" (R. Lionberger, 2013) referring to our article.

Overall, the dissertation provides significant contributions to societies. The conclusions and results of the conducted investigations are not only applicable for drug regulatory authorities, but also to patients and healthcare professionals. Indirectly it reduces the financial burden of the healthcare system and also promote better communications between the stakeholders. 
